\title{
Stability Analysis of a Hybrid Modular Multilevel Voltage Source Converter
}

\author{
By: \\ Charithri Arunika Nanayakkara Yapa
}

A Thesis submitted to the Faculty of Graduate Studies of The University of Manitoba in partial fulfilment of the requirements of the degree of

MASTER OF SCIENCE

Department of Electrical and Computer Engineering

University of Manitoba

Winnipeg

Copyright (C) 2018 by Charithri Arunika Nanayakkara Yapa 
To my parents and Dulshan. 


\section{Acknowledgements}

My sincere gratitude goes to my advisor, Prof. Udaya D. Annakkage and coadvisor, Prof. Shaahin Filizadeh, for their continuous support, advice, guidance and encouragement given to me throughout the thesis research. I consider it as a great privilege for having had an opportunity to work with them and their knowledge and experience shared with me were undoubtedly valuable in making a significant contribution through my thesis. Further I would like to thank Dr. C. Karawita for sharing his views and comments in making the outcomes of the thesis more relevant and applicable to the practical scenarios.

I must also thank all the academic, administrative and technical staff of the Department of Electrical and Computer Engineering, especially Amy Dario, Traci Gledhill and Erwin Dirks for their assistance.

I would like to acknowledge the University of Manitoba and the Government of Manitoba for the financial supports received in the forms of Graduate Fellowships and Graduate Scholarships.

I would like to sincerely thank my collogues, Akbo Rupasinghe and Curtis Shumski for the valuable advise and guidance given to me from the beginning to the end of this thesis. Further I would like to extend my sincere gratitude towards all my friends in the Department of Electrical and Computer Engineering for encouraging me and making my experience as a graduate student in University of Manitoba, a memorable one. I would also like to thank my Sri Lankan friends living in Winnipeg, for supporting me and making my stay in Canada comfortable.

Last but not the least, my heartfelt gratitude goes to my dear parents and grandmother for encouraging me during the hard times throughout these years. Finally 
I would like to sincerely thank my husband Dulshan, for his patience, understanding, support and encouragement which was a great motivation in completing by graduate studies in Canada. 


\begin{abstract}
Hybrid Multilevel Converter topologies have been gaining attention in the area of High Voltage Direct Current (HVDC) transmission using Voltage Source Converters (VSCs) mainly due to their improved output voltage quality, resiliency to ac and dc faults, lower converter losses and smaller converter-footprint when compared with the conventional multilevel and modular multilevel configurations. Among these different hybrid converters the configuration studied in this thesis research is a novel low-loss VSC topology which combines H-bridge converters that are soft-switched with cascaded strings of multilevel cells, providing the wave-shaping function. This particular arrangement enables reduction of switching losses in the converter and utilizes a triplen harmonic addition modulation scheme for voltage control.
\end{abstract}

The stability evaluation of the selected converter configuration is the prime objective of this thesis research. A linearized small-signal model is developed and the 'stability in small' is evaluated by applying small perturbations to the active and reactive power references of the converter. The validation of the developed model is performed against a detailed non-linear Electro-Magnetic Transient (EMT) simulation model.

The stability criterion based on Lyapunov's first method has been applied on eigenvalues (modes) which are the frequency domain attributes obtained from the developed state-space model. Further the impact of the system strength parametrized by the short-circuit ratio on the stability and performance of the converter has been analysed by selecting two case studies that focus on a strong and a weak ac network connected to the converter. The sensitivity of the modes that define the stability of the converter, for the variation in Phase-Locked Loop (PLL) gains 
and measurement delays of instantaneous voltages and currents of the decoupled $d q$ control scheme have been studied which provides a better insight to its performance.

The power handling capability of this converter topology has been analysed which is an attribute that describes the applicability of this configuration as a prospective candidate for long-distance power transmission using HVDC VSC schemes. The restrains imposed on the power transfer of the converter by the topology related constraints along with the network attributes such as the system strength are studied in this analysis.

In general, this thesis evaluates stability of the converter, factors that have an impact on instabilities and the power carrying capability restrained by the system strength and converter limitations of a novel hybrid modular multilevel voltage source converter topology. This provides an insight to the viability of implementing the configuration in long distance HVDC transmission, with varying ac network conditions. 


\title{
List of Symbols
}

\author{
$t$ \\ $\omega$ \\ $\omega_{0}$ \\ $v_{t}, i_{t}$ \\ $\widehat{v}_{t}$ \\ $\vec{V}$ \\ $v_{s}$ \\ $P_{x}, Q_{x}$ \\ $V_{x}, I_{x}$ \\ $v_{t a}, v_{t a}^{\prime}$ \\ $v_{C L}^{a}, v_{C L}^{b}, v_{C L}^{c}$ \\ $V_{G}$ \\ $v_{d c}, i_{d c}$ \\ $R_{s}, L_{s}$ \\ $L$ \\ $R_{d c}, L_{d c}$ \\ $C_{\text {cell }}, v_{\text {cap }}$ \\ $n_{c}$ \\ $\delta$ \\ $\delta_{m}$ \\ $K_{P}, K_{I}$ \\ $T$ \\ $\lambda$ \\ $f, \zeta$ \\ Time \\ Angular frequency of the power system \\ Base angular frequency of the power system \\ Instantaneous voltage and current at the converter terminal \\ Peak value of the terminal voltage \\ Vector form of voltage $V$ \\ Thevenin Equivalent Voltage \\ RMS values of active power and reactive power \\ Phasor quantities of $v, i$ \\ Instantaneous values of voltage of phase a of ac side and converter side \\ of the tranformer \\ Instantaneous voltages synthesized by chain-link converters \\ of phase $a$, phase $b$ and phase $c$ \\ DC-link voltage \\ Composite voltage across the chain-link converters and dc current \\ $R$ and $L$ values of the Thevenin equivalent impedance \\ De-coupling inductance of vector-control system; ideally $L=L_{s}$ \\ DC line resistance and filter inductance \\ Submodule cell capacitance and instantaneous cell voltage \\ Number of submodules per phase \\ Actual phase angle of $v_{s}$ \\ Estimated phase angle of $v_{s}$ \\ Proportional and integral gains of a PI controller \\ Time constant of a single pole delay block \\ Eigenvalue \\ Frequency and damping of the eigenvalue (mode)


Subscript (ref) Reference value

Subscript $(m) \quad$ Measured value

Subscripts $R, I \quad$ Real and imaginary components

Subscripts $d, q$ Direct and quadrature components

Subscript $0 \quad$ Initial value 


\section{Abbreviations}

HVDC High Voltage Direct Current

HVAC High Voltage Alternating Current

CSC Current Source Converter

LCC Line Commutated Converter

IGBT Insulated Gate Bi-polar Transistor

PWM Pulse Width Modulation

VSC Voltage Source Converter

MMC Modular Multilevel Converter

HB Half Bridge

FB Full Bridge

HMC Hybrid Multilevel Converter

PCC Point of Common Coupling

AAC Alternate Arm Converter

EMTDC Electro-Magnetic Transients including DC

RMS Root Mean Squared

PI Propotional - Integral

SRF-PLL Synchronous Reference Frame-Phase Locked Loop

MI Modulation Index

SCR Short Circuit Ratio

FFT Fast Fourier Transform

SSO Sub Synchronous Oscillations

PF Power - Factor 


\section{Contents}

Acknowledgements ii

Abstract

List of Symbols $\quad$ vi

Abbreviations viii

$\begin{array}{ll}\text { Contents } & \text { ix }\end{array}$

List of Tables $\quad$ xiii

List of Figures $\quad$ xiv

1 Introduction $\quad 1$

1.1 Background . . . . . . . . . . . . . . . . . . . 1

1.2 Developments involving VSC-HVDC technology . . . . . . . . . 2

1.3 Development of Hybrid VSC Topologies . . . . . . . . . . . . . . . 3

1.3.1 HMC with AC side Cascaded H-bridge Cells . . . . . . . . . 4

1.3.2 HMC with DC side Cascaded Cells . . . . . . . . . . . . 5

1.3.3 Alternate Arm Converter (AAC) . . . . . . . . . 6

1.3.4 HMC with Half or Full-Bridge Cells across the DC Link . . 7

1.3.4.1 Converter Topology and Basic Operation . . . . . . 8

1.3.4.2 Voltage Control using Triplen Harmonic Injection . 12 
1.4 Research Objectives . . . . . . . . . . . . . . . . . . . . . 14

1.5 Thesis Outline . . . . . . . . . . . . . . . . 16

2 Modelling of the Hybrid Modular Multilevel VSC 18

2.1 Electro-magnetic Transient Modelling of the Test System . . . . . . 19

2.2 Development of Linearized Small-signal Model . . . . . . . . . . . . 20

2.2 .1 AC System Model . . . . . . . . . . . . . . . . . . . 22

2.2.2 Phase-Locked Loop Model . . . . . . . . . . . . . . . . 24

2.2.3 Measurement Filter Model . . . . . . . . . . . . . . . . 26

2.2.4 Axis Transformation . . . . . . . . . . . . . . . 28

2.2.5 Control System Model . . . . . . . . . . . . . 30

2.2.5.1 Small-signal Model of the AC Control System . . . 31

2.2.5.2 Small-signal Model of the DC Control System . . . 35

2.2.6 DC System Model . . . . . . . . . . . . . . . . . 39

2.2.7 Overall State-Space Model of the Hybrid Modular Multilevel

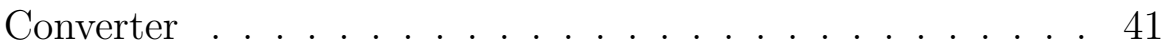

2.3 Validation of the Overall State-Space Model of the Converter . . . . 44

2.3.1 Disturbance applied to the active power reference . . . . . . 45

2.3.2 Disturbance applied to the reactive power reference . . . . . 46

2.4 Modified Overall State-Space Model of the Hybrid Modular Multilevel Converter . . . . . . . . . . . . . . . . . . . . 47

2.4.1 Validation of the Modified Small-Signal Model of the Converter 50

2.4.1.1 Disturbance applied to the active power reference . 50

2.4.1.2 Disturbance applied to the reactive power reference 50

3 Stability Analysis of the Hybrid Modular Multilevel VSC 53

3.1 Eigenproperties and Stability Criterion . . . . . . . . . . 54

3.1 .1 Eigenvalues . . . . . . . . . . . . . . . 54

3.1 .2 Eigenvectors . . . . . . . . . . . . . . 56

3.1.3 Participation Factor . . . . . . . . . . . . 57 
3.2 Eigenvalue Analysis of the Test System . . . . . . . . . . . . . . . 57

3.2.1 Analysing the impact of the system strength on the stability of the converter . . . . . . . . . . . . . . . 58

3.2.2 Participation Factor Analysis . . . . . . . . . . . . 60

3.3 Sensitivity of the Small-Signal Stability of the Converter to Controller Gains . . . . . . . . . . . . . . . . . . . . . . . . 64

3.3 .1 Impact of the PLL gains . . . . . . . . . . . . . . . . . 64

3.3.2 Sensitivity to measurement delays of $d q$ voltages and currents 66

3.4 Impact of Transformer Saturation on Converter Performance with Low SCR . . . . . . . . . . . . . . . 76

4 Power Transfer Capability of the Hybrid Multilevel Converter 82

4.1 Calculation of the active power delivered by the converter . . . . . . 84

4.2 Constraints imposed on maximum power deliverable by the converter 85

4.2.1 Limits imposed by the maximum current through the converter 86

4.2.2 Limits imposed by the converter voltage . . . . . . . . . 86

4.2.3 Impact of the system strength on the power carrying capability of the converter . . . . . . . . . . . 87

4.3 Evaluation of power handling capability for different mode of converter operation . . . . . . . . . . . . . . . . . 88

4.3.1 Power handling capability of the converter with constant reactive power supply . . . . . . . . . . . . 88

4.3.2 Power handling capability with reactive power absorbed by the converter . . . . . . . . . . . . . . . 92

4.3.3 Power handling capability of the converter with constant power factor operation . . . . . . . . . . . . . 94

5 Conclusions, Contributions and Future Work 98

5.1 Conclusions . . . . . . . . . . . . . . . . . . . . . . . . . . . . . . 98

5.2 Contributions . . . . . . . . . . . . . . . . 100 
5.3 Future Works . . . . . . . . . . . . . . . . . . . . . 101

Bibliography

Appendix A 


\section{List of Tables}

2.1 Simulation Parameters . . . . . . . . . . . . . . . . . . 19

2.2 State variables of the small-signal model of the converter . . . . . . 42

2.3 Control inputs of the small-signal model of the converter . . . . . . 42

2.4 PI controller gains of the test system . . . . . . . . . . . . . . 44

3.1 Oscillatory modes for ac system strengths of $\mathrm{SCR}=4.0$ and $\mathrm{SCR}=2.059$

3.2 Order of states of the small-signal model . . . . . . . . . . . . . . 61

3.3 Classification of modes for $\mathrm{SCR}=4.0 \ldots \ldots$. . . . . . . . . 62

3.4 Classification of modes for $\mathrm{SCR}=2.0 \ldots \ldots \ldots$. . . . . . . 62

3.5 Time constants of the measurement filters of outer and inner-loop controls . . . . . . . . . . . . . . . . . . . . 69

3.6 Order of states for the modified converter small-signal model to include measurement delays . . . . . . . . . . . . . . . 70

3.7 Oscillatory modes of the modified state-space model with the converter connected to a moderately strong ac system . . . . . . . . . . 72

3.8 Oscillatory modes of the modified state-space model with the converter connected to a weak ac system . . . . . . . . . . . . 74

4.1 Test system parameters . . . . . . . . . . . . . . . . . 89 


\section{List of Figures}

1.1 Three phase circuit arrangement of the hybrid modular multilevel $\mathrm{VSC} \ldots \ldots \ldots \ldots \ldots \ldots \ldots$

2.1 Schematic of the test system for the hybrid modular multilevel VSC 19

2.2 PLL block diagram . . . . . . . . . . . . . . . . . 25

2.3 Measurement filter block . . . . . . . . . . . . . . . . 26

2.4 RI- $d q$ transformation . . . . . . . . . . . . . . . . . . . . . . 28

2.5 Decoupled $d q$ control scheme . . . . . . . . . . . . . . . . . . . . . 30

2.6 PI controller block . . . . . . . . . . . . . . . . . . . . 32

2.7 Capacitor voltage control scheme (outer-loop) . . . . . . . . . 37

2.8 DC link current control scheme (inner-loop) . . . . . . . . . . . 37

2.9 Equivalent circuit of the DC system . . . . . . . . . . . . . . . 39

$2.1015^{\text {th }}$ order small signal model of the hybrid modular multilevel VSC 43

2.11 Variation in active power $\left(\Delta P_{(a c)}\right)$ for a small perturbation in respective reference $P_{(r e f)} \ldots \ldots \ldots \ldots$

2.12 Variation in reactive power $\left(\Delta Q_{(a c)}\right)$ for a small perturbation in respective reference $Q_{(r e f)} \ldots \ldots \ldots$. . . . . . . . . 46

2.13 Modified small signal model of the hybrid modular multilevel VSC . 49

2.14 Variation in active power $\left(\Delta P_{(a c)}\right)$ for a small perturbation in respective reference $P_{(r e f)} \ldots \ldots \ldots \ldots \ldots$

2.15 Variation in reactive power $\left(\Delta Q_{(a c)}\right)$ for a small perturbation in respective reference $Q_{(r e f)} \ldots \ldots \ldots \ldots$ 
3.1 Comparison of results for the converter connected to a moderately strong ac system $(\mathrm{SCR}=4.0)$ (I) Variation in active power for a small disturbance in $P_{(r e f)}$ (II) Variation in reactive power for a small disturbance in $Q_{(r e f)} \ldots \ldots$. . . . . . . . . . . . 59

3.2 Comparison of results for the converter connected to a moderately strong ac system $(\mathrm{SCR}=2.0)$ (I) Variation in active power for a small disturbance in $P_{(r e f)}$ (II) Variation in reactive power for a small disturbance in $Q_{(r e f)} \ldots \ldots$. . . . . . . . . . . 60

3.3 Participation factors of oscillatory modes for $\mathrm{SCR}=4.0$. . . . . . . 61

3.4 Participation factors of oscillatory modes for $\mathrm{SCR}=2.0$. . . . . . . 63

3.5 Trajectories of critical modes (second mode enlarged) for a change in PLL gains with moderately high SCR (=4.0) of the connected ac

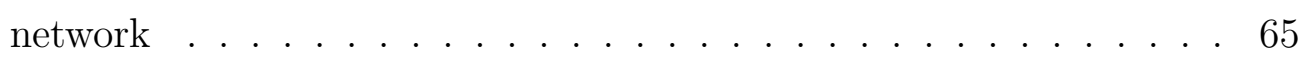

3.6 Trajectories of critical modes (second mode enlarged) for a change in PLL gains with weak SCR (=2.0) of the connected ac network . 65

3.7 Measurement filter block . . . . . . . . . . . . . . . 67

3.8 Inner-loop control with measurement delays included . . . . . . . 68

3.9 Modified $19^{\text {th }}$ order small signal model of the hybrid modular multilevel VSC . . . . . . . . . . . . . . . . 71

3.10 Participation factors of the oscillatory mode for $\mathrm{SCR}=4.0$. . . . . 73

3.11 Participation factors of the oscillatory mode for $\mathrm{SCR}=2.0$. . . . . 75

3.12 Variation in active power $\left(\Delta P_{(a c)}\right)$ for a small perturbation in respective reference $P_{(r e f)}$ with the turns ratio of the transformer between ac side to converter side set to $1: 1.1 \ldots \ldots$. . . . . 76

3.13 Low frequency oscillatory component of the variation in reactive power $\left(Q_{(a c)}\right)$ in steady state with the turns ratio of the transformer between ac side to converter side set to $1: 1.1 \ldots \ldots 77$ 
3.14 Comparison of results for the turns ratio of the transformer changed to $1: 1$ between ac side and converter side (I) a small disturbance applied to $P_{(r e f)}(\mathrm{II})$ a small disturbance applied to $Q_{(r e f)}$. . . . . 79

3.15 Comparison of results for the turns ratio of the transformer changed to 1.1:1 between ac side and converter side (I) a small disturbance applied to $P_{(r e f)}(\mathrm{II})$ a small disturbance applied to $Q_{(r e f)} \ldots$. . . 79

3.16 Comparison of results for $\mathrm{SCR}=4.0$ (I) a small disturbance applied to $P_{(r e f)}(\mathrm{II})$ a small disturbance applied to $Q_{(r e f)} \ldots \ldots . . . .80$

4.1 Schematic of the test system for the hybrid modular multilevel VSC 83

4.2 Variation of $V_{t}$ with active power delivered for a converter connected to a strong ac network with $\mathrm{SCR}=4.0$; constant $Q$ operation . . . . 90

4.3 Variation of $V_{t}$ with active power delivered for a converter connected to a weak ac network with $\mathrm{SCR}=2.0$; constant $Q$ operation . . . . 91

4.4 Comparison of results for $P_{\max } \ldots \ldots \ldots$. . . . . . . . . . 92

4.5 Variation of $V_{t}$ with active power delivered for a converter connected to a strong ac network with $\mathrm{SCR}=4.0 ; Q$ absorbed from the network 93

4.6 Variation of $V_{t}$ with active power delivered for a converter connected to a weak ac network with $\mathrm{SCR}=2.0 ; Q$ absorbed from the network 94

4.7 Variation of $V_{t}$ with active power delivered for a converter connected to a strong ac network with $\mathrm{SCR}=4.0$; constant $\mathrm{PF}$ operation . . . 95

4.8 Variation of $V_{t}$ with active power delivered for a converter connected to a weak ac network with $\mathrm{SCR}=2.0$; constant PF operation . . . . 96 


\section{Chapter 1}

\section{Introduction}

\subsection{Background}

High Voltage Direct Current (HVDC) transmission has gained attention in the current Power System configuration, evolving through decades to overcome the engineering and reliability challenges that arise in long distance power transmission using High Voltage Alternating Current (HVAC). Various factors drive the emergence of Distributed Generation including the need for reducing green house gas emissions from electricity generation which has been identified as the major contributor of global temperature rise, utilization of waste energy resources, fuel diversification have created a requirement to facilitate the grid integration of renewable energy resources including wind and solar Photovoltaic (PV). In such situations, HVDC plays an important role in accommodating this large penetration of renewable power such as offshore wind farms to the main power system. This otherwise would be challenging without causing stability issues in the connected ac system due to the intermittent nature of these technologies. 
Early HVDC transmission schemes were on the basis of Current Source Converters (CSC) incorporating mercury arc valves later replaced with thyristors, referred to as Line Commutated Converters (LCC). Besides better power ratings with higher efficiency, these converters are vulnerable to ac short circuits with their inherent commutation failure problem. Moreover, LCC requires the support of connected ac network for its operation hence inhibits black start of the converter as well as integration with weak ac grids. Voltage Source Converter- High Voltage DC (VSCHVDC) topologies hence gained attraction over conventional CSCs favoured by the semiconductor development. This further provided benefits including independent control of active and reactive power, provision of voltage support to weak ac grids, ac side short-circuit proof feature and improved power quality performance with minimal requirement of filtering. Further the VSC configuration facilitates the implementation of multi-terminal HVDC schemes since reversal of flow does not result in a reversal of the polarity of the DC voltage [1] [2] [3] [4]. VSC-HVDC technology has enabled connection of off-shore wind generation to the mainland power system using HVDC links, overcoming the stability constraints in HVAC transmission.

\subsection{Developments involving VSC-HVDC tech- nology}

VSC-HVDC technology has been an extensively discussed research topic with the interest of improving the power ratings of the converters making it a competitive candidate in high power DC applications while minimizing switching losses hence improving the converter efficiency. These developments have evolved the VSCs from its basic form of two-levels which employ a large number of series connected 
Insulated-Gate Bipolar Transistors (IGBTs) to multilevel converter configurations and most recent development of Modular Multilevel Converters (MMC) [5]. The former group of converter is referred to as controllable switch type VSCs and uses high frequency Pulse Width Modulation (PWM) techniques. The latter group of converters also referred to as controllable voltage source type VSC is gaining attention in the current context of HVDC power transmission which is aimed at improved ac side waveform quality to eliminate the harmonic filter requirement and improved converter efficiency with smaller converter footprint and overall cost. Additionally the low $\frac{d v}{d t}$ enables the use of transformers with less insulation requirement [1] [6] [7].

The MMC topology incorporates hundreds of independently controllable HalfBridge (HB) or Full-Bridge (FB) cells per arm, referred to as submodules which generate a staircase voltage that closely approximate the sinusoidal waveform with low harmonic distortion. Further this configuration offers the advantage of reduced switching losses since it does not rely on high frequency PWM and scalability due to the modular design which makes it easy to handle high voltage applications [8].

\subsection{Development of Hybrid VSC Topologies}

Extensive research is being conducted on further optimization of the key performance indices of VSC-HVDC schemes which mainly include output voltage quality, resiliency to ac and dc faults, and lower conversion losses hence improved conversion efficiency [9]. Hybrid converters that combine the advantages of conventional two-level VSC configuration with the modular multilevel topology have gained interest at the present and novel converter configurations are being developed for this purpose. Current research has been mainly focused on hybrid converter topologies 
which include Hybrid Multilevel Converter (HMC) with ac side cascaded H-bridge cells [3] [5] [6] [7], HMC with dc side cascaded cells [9] [10] [11], alternate arm converter [1] [2] [8] [11] and HMC with half or full bridge cascaded cells connected across the dc link [4] [11] [12].

\subsubsection{HMC with AC side Cascaded H-bridge Cells}

The topology consists of two main developments different from one another depending on the main power stage of the converter, which is responsible for the control of the voltage at the terminals and power transfer between ac and dc sides. The wave-shaping circuit which is the low power stage comprising of series connected FB submodules acts as an active filter stage and attenuates the harmonic voltages generated by the main power stage. The main power stage is switched at low frequency in order to minimize losses in the converter.

One configuration out of the two utilizes the conventional two-level VSC as its main power stage which is connected to the ac terminal with series connected cascaded FB cells [3] [5]. This configuration has a smaller converter footprint compared to other hybrid topologies and utilizes one fourth of the number of FB submodules compared to the equivalent modular multilevel converter configuration. Further the converter is also capable of blocking dc-faults. The second uses cascaded HB cells in main power stage, connected in series with FB cells of a similar arrangement to the previous topology [6] [7].

The former has several drawbacks which include high voltage stresses in switches of the two-level VSC and high inrush current from the ac network when the DC side capacitors charge, following a pole-to-pole short-circuit of DC terminals [6] [9]. Although, this inrush current in the restoration stage following a fault condition 
is less compared to that of an Alternate Arm Converter (AAC); an alternative topology which is discussed in a latter part of this section. The main power stage generates an output voltage of only two levels thus contains a higher content harmonics which has to be filtered by the cascaded FB cells. This would also require tuned filters to eliminate $5^{\text {th }}$ and $7^{\text {th }}$ harmonics that might arise due to the mis-synchronization of the two power stages [6] [9]. The two-level converter being the low power stage should be capable of tracking the rate of change of voltage for proper synchronization with its active filter stage. [6].

The latter configuration thus utilizes a cascaded HB cell MMC arrangement as the main power stage which is responsible for the control of magnitude and phase of the fundamental voltage at the Point of Common Coupling (PCC) thus regulating active and reactive power requirements of the converter connected ac system. The cascaded series FB cell arrangement acts as a low power stage which attenuates the harmonics of the main power stage, providing active filtering. This converter has achieved reduced conversion losses compared to MMC configuration with FB cells. Further the HB cells provide controlled re-charging of the dc cable capacitance followed by a dc side fault clearance while the FB cells remain blocked [6].

\subsubsection{HMC with DC side Cascaded Cells}

A single phase of this converter topology combines a H-bridge converter with cascaded FB cells connected in series at the dc terminals [9] [10] [11]. The Hbridge converter comprises of series connected strings of IGBTs. The overall rating of the VSC is shared between the series IGBT valves and multilevel converter thus a fewer number of cells would be required. This is advantageous since the converter has a smaller footprint due to reduced number of cell capacitors which is a governing factor of the size and weight of the submodule. Further this converter 
is inherently capable of blocking dc-faults due to the series connected FB cells where the cell capacitors would provide an opposing voltage to the ac side voltage thereby eliminating the ac current contribution to the fault current during a shortcircuit across the dc terminals. The ability to generate negative voltages enable this converter to continue operation while being connected to the network during a fault in the dc side even though the dc voltage has collapsed.

The H-bridge converter which is the main power stage of this converter topology is switched at fundamental frequency and near zero voltage. The converter hence has achieved a reduction in converter losses due to switching and performs with improved efficiency.

\subsubsection{Alternate Arm Converter (AAC)}

The AAC is a hybrid between a modular multilevel converter comprising of FB cells and the two-level configuration in the form of series connected IGBT valves referred to as the 'director switch'. Further, this topology could be considered as a combination of the conventional CSC which uses switches to direct the phase currents to upper or lower arm. The FB cells that construct the multilevel topology is responsible for constructing the converter voltage from that of the dc-link [1] [2] [8] [11].

This configuration requires half the number of $\mathrm{FB}$ cells compared to the $\mathrm{HB}$ or FB MMC configuration but the twice the number when compared with the HMC with ac side cascaded cells. The series connected IGBTs are soft switched in order to reduce semiconductor losses thereby making the topology more efficient.

The upper and lower arm of each converter phase is operated in a complementary manner and operates for half the time of the fundamental period while blocking 
the full dc voltage during the other half period. Successful current commutation between the upper and lower arms is achieved by introducing an overlap period around the zero voltage level. This alternating operation of the converter arms reduces the switching losses, which is less than that of the HMC with ac-side cascaded cells, which has a smaller footprint compared to the AAC. Further, with the presence of FB cells in each arm the converter could control the phase current during the a collapse of dc side voltage hence posses the capability of dc fault ride-through.

The major drawback of this converter configuration has been identified to be the large inrush current the converter arm experiences during the overlap period. Further the series connected IGBTs should be provided with switching characteristics that are identical with equal voltage sharing between the semiconductor devices which is a disadvantage of this topology. Although this converter is resilient to dc-side faults, restoring the submodule capacitor voltages to their pre-fault value while recovering from a short circuit across the dc terminals is slower than a conventional MMC and HMC with ac side cascaded cells.

\subsubsection{HMC with Half or Full-Bridge Cells across the DC Link}

A single phase of this novel hybrid converter configuration combines a chain-link of cascaded $\mathrm{HB}$ or FB submodules at the dc terminals of a H-bridge arrangement. The chain-link converters provide the wave-shaping function which generates a rectified sinusoid of the desired ac side voltage from several levels of dc voltage. The H-bridge switches 'direct' this synthesized voltage to the converter connected ac network and comprise of series connected IGBT valves which reduce the voltage stresses on an individual switch. The chain-link converters are switched using a 
traditional multilevel modulation strategy, synthesizing a rectified sinusoidal voltage waveform repeating twice a cycle. This enables the series connected switches of the H-bridge to be switched at fundamental frequency and zero point of the voltage imposed on them. [4] [11].

Such arrangement enables the chain-link converters, where the hard switching occur to be connected outside of the main power path thus operating at only a fraction of the line current while the main power stage that comprises of $\mathrm{H}$ bridges to be soft switched at fundamental frequency. Further, the overall rating of the converter is being shared between the series connected H-bridge IGBT valves and the chain-link cells. Thus this particular converter configuration associates with less switching losses and at the same time less ratings for the submodules when compared to the alternative hybrid converter configurations discussed in this section [11]. This is advantageous in terms of increasing the conversion efficiency and at the same time to have a smaller converter footprint, making this topology an ideal candidate for long distance high power transmission and connecting off-shore wind generation to the inland power system. In comparison with HMC with ac side cascaded cells which is the widely adapted hybrid converter topology having a small footprint, this configuration has the same number of cells per arm as the former for the same ac side and dc-link voltages whereas minimal switching losses compared to that. This thesis is based on the converter configuration of HMC with half-bridge cells connected across the DC link and the following sections provides a brief introduction to the topology and the basic operation of the converter.

\subsubsection{Converter Topology and Basic Operation}

The schematic of the converter considered is given in Fig. 1.1. A phase of the converter comprises of cascaded half-bridge submodules connected across the dc 


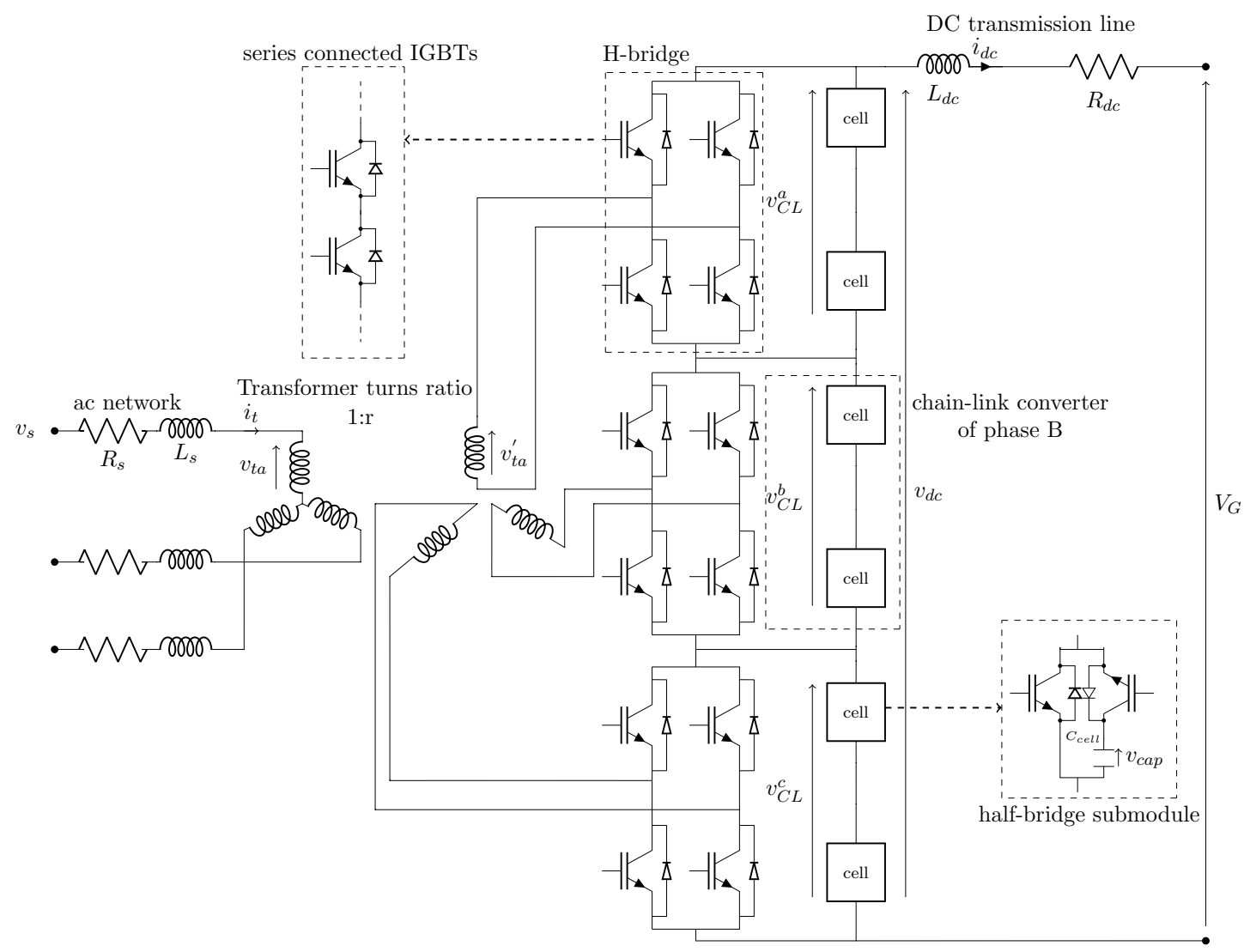

Figure 1.1: Three phase circuit arrangement of the hybrid modular multilevel VSC; redrawn from Fig.1 in $[4]^{1}$

terminals of an H-bridge arrangement, referred to as the chain-link converters. These chain-link converters provide the wave-shaping function and synthesize a rectified sinusoid of the required ac side demand in voltage steps. The H-bridge arrangement which comprises of series connected IGBT valves unfolds the negative cycle of this generated voltage imposed on its dc terminals and 'directs' to the converter connected ac network. Three of these single phase converters are cascaded in series at their dc terminals to develop a three phase converter as illustrated in Fig. 1.1. The transformer provides isolation between the ac side and

\footnotetext{
${ }^{1}$ C 2013 IEEE
} 
the converter side.

For the expected operation of the multilevel converters it is necessary to have equally distributed charges hence capacitor voltages among the chain-link converter cells which is achieved through a suitable balancing algorithm. A simple capacitor balancing algorithm has been incorporated in this topology which considers the polarity of the phase current, voltage of the capacitors and the target ac side voltage to be synthesized by the cells thereby rotating its position in the waveform following a sorting mechanism [13]. A proper capacitor balancing algorithm ensures that at steady state there will be no net capacitor charging or discharging therefore the active power exchange with the chain-link converter cells are sufficient to supply only the losses [4].

With a 1:r transformer ratio between the ac side and the converter side, the three phase voltages appearing on the ac terminals of the converter could be expressed in the form given in Equation (1.1).

$$
v_{t x}=\frac{1}{r} \widehat{v}_{t}\left(\sin \left(\omega t-\frac{2 \pi n}{3}\right)\right) \quad \text { for }(x, n)=\{(a, 0),(b, 1),(c, 2)\}
$$

The voltage appearing at the converter side of the transformer is hence given by the Equation (1.2).

$$
v_{t x}^{\prime}=\widehat{v}_{t}\left(\sin \left(\omega t-\frac{2 \pi n}{3}\right)\right) \quad \text { for }(x, n)=\{(a, 0),(b, 1),(c, 2)\}
$$

Thus the voltage to be synthesized by the chain-link converters and applied at the dc terminals of the H-bridge converters is expressed in the form given in Equation (1.3).

$$
v_{C L}^{x}=\widehat{v}_{t}\left|\sin \left(\omega t-\frac{2 \pi n}{3}\right)\right| \quad f o r(x, n)=\{(a, 0),(b, 1),(c, 2)\}
$$


where $\widehat{v_{t}}$ refers to the peak converter voltage which is an output of the ac side converter control scheme obtained by $\widehat{v}_{t}=\sqrt{v_{t d}^{2}+v_{t q}^{2}}$. The chain-link converters are modulated with the reference signals given in Equation (1.3); utilizing a traditional multilevel modulation scheme [4] [14] .

As shown in Fig. 1.1, the dc-link voltage $v_{d c}$ is the composite chain-link phase voltages as obtained from Equation (1.4).

$$
v_{d c}=v_{C L}^{a}+v_{C L}^{b}+v_{C L}^{c}
$$

The mean chain-link phase voltage $\bar{v}_{C L}$ can be obtained using Equation (1.3) and is given by Equation (1.5) from which the mean composite chain-link voltage $\bar{v}_{d c}$ can be obtained as given in Equation (1.6).

$$
\begin{gathered}
\bar{v}_{C L}=\frac{2 \widehat{v}_{t}}{\pi} \\
\bar{v}_{d c}=\frac{6 \widehat{v}_{t}}{\pi}
\end{gathered}
$$

In steady state $\bar{v}_{d c}$ is equal to the dc grid voltage $V_{G}$. The modulation index $(M I)$ of the converter can be defined by following the convention for two-level VSCs which is given in Equation (1.7).

$$
M I=\frac{\widehat{v}_{t}}{\bar{v}_{d c} / 2}
$$

The above equation can be simplified by substituting for $\bar{v}_{d c}$ from Equation (1.6) and is given by Equation (1.8).

$$
M I=\frac{2 \pi}{6} \approx 1.05
$$




\subsubsection{Voltage Control using Triplen Harmonic Injection}

Equation (1.8) reveals that the $M I$ of the converter is fixed for the above explained mode of operation in which the ratio between the ac-side voltage and dc side voltage is a constant for a specified local grid voltage $V_{G}$. This becomes an unfavourable condition for voltage control of the converter connected ac network. Since the converter voltage magnitude $\widehat{v}_{t}$ cannot be varied without affecting the dc-side voltage, this inhibits the reactive power compensation capability of the converter. Thus [4] proposes a voltage control technique to overcome this constraint of the converter configuration, while enabling the H-bridge arrangement to be maintained soft-switched. This does not incorporate high frequency PWM techniques to modify the desired ac-side voltage thereby maintaining minimal switching losses which has been emphasized as a main advantage of this topology over its alternative configurations. The methodology presented incorporates modifying of the target ac voltage by the addition of triplen harmonics. Through this mechanism the ratio of mean rectified ac-side voltage to the fundamental is altered.

The case of third harmonic voltage injection has been considered hence the chainlink converters are modulated using reference signals which comprise of the fundamental sinusoid modified by adding the third harmonic voltage component. This third harmonic voltage added provides a dc component of voltage that can be controlled to positively or negatively compensate for the changes in the rectified ac voltage reference over each half cycle. This enables the variation of the ac-side voltage magnitude in the presence of fixed dc-link voltage which was not possible with the basic control configuration explained in section 1.3.4.1. Further the triplen harmonics comprise only of the zero sequence component thus will not appear in the line-to-line voltage on the ac side. This is advantageous since the H-bridge converters are still allowed to be switched at line-frequency on the zero 
voltage imposed on them without adding distortions to the line-voltages.

The modified ac voltage at the converter side of the transformer with third harmonic compensation is thus given by Equation (1.9) where $\alpha_{3}$ denotes the magnitude of the third harmonic compensation added. The amount of triplen harmonic compensation to be added is obtained through a cascaded arrangement of PI controllers which has been explained in section 2.2.5.2.

$$
v_{t x}^{\prime}=\widehat{v}_{t}\left[\sin \left(\omega t-\frac{2 \pi n}{3}\right)+\alpha_{3} \sin (3 \omega t)\right] \quad \text { for }(x, n)=\{(a, 0),(b, 1),(c, 2)\}
$$

Hence the phase voltages to be synthesized by the chain-link converters are given in Equation (1.10) which will be used as the modulating signal for the HB submodules.

$$
v_{C L}^{x}=\widehat{v}_{t}\left|\sin \left(\omega t-\frac{2 \pi n}{3}\right)+\alpha_{3} \sin (3 \omega t)\right| \quad \text { for }(x, n)=\{(a, 0),(b, 1),(c, 2)\}
$$

With the addition of the third harmonic voltage to the desired ac-side fundamental sinusoid, the mean chain-link phase voltage $\bar{v}_{C L}$ and the mean composite chain-link voltage $\bar{v}_{d c}$ will be modified as given in Equation (1.11) and (1.12) respectively.

$$
\begin{aligned}
\bar{v}_{C L} & =\frac{2 \widehat{v}_{t}}{\pi}\left(1+\alpha_{3} / 3\right) \\
\bar{v}_{d c} & =\frac{6 \widehat{v}_{t}}{\pi}\left(1+\alpha_{3} / 3\right)
\end{aligned}
$$

As given from Equation (1.12), the magnitude of the ac-side voltage can be controlled continuously by adjusting the magnitude of the third harmonic voltage compensation $\alpha_{3}$ while maintaining a constant mean composite chain-link voltage $\bar{v}_{d c}$. Thus the modulation index will now be modified as expressed in Equation (1.13). This further explains the ability to vary the modulation index thereby the converter voltage magnitude $\widehat{v}_{t}$ for regulating the reactive power exchange with 
the converter connected ac network, at the presence of a constant dc side voltage.

$$
M I=\frac{2 \pi}{6\left(1+\alpha_{3} / 3\right)}
$$

The voltage generated by the half-bridge submodules is of magnitude 0 or $v_{c a p}$ whereas it is not capable of generating negative voltages. Hence the allowable third harmonic compensation magnitude is restricted to a range given in Equation (1.14) $[15]$.

$$
-1 / 3 \leq \alpha_{3} \leq 1
$$

Therefore applying the above limits to Equation (1.13) yields the allowable range for the modulation index of the converter controls as given in Equation (1.15).

$$
0.785 \leq M I \leq 1.178
$$

\subsection{Research Objectives}

This thesis is based on a novel hybrid modular multilevel converter topology which is a prospective candidate for HVDC transmission using voltage source converters. This configuration improves performance indices of a converter such as quality of the output voltage and current, reduces converter losses hence improves efficiency and modularity, scalability are offered at a smaller converter footprint. The motivation behind this thesis was to asses the converter's 'stability in small'. This evaluates the controls related stability issues which might cause implications on the large power system to which the converter is connected to, followed by a small disturbance. 
Hybrid converter configurations are gaining attention over conventional multilevel VSCs such as the regular MMC topology thus extensive research is being conducted in order to analyse the performance of these novel converters. This includes evaluating their viability to be adopted in bulk power de transmission over long distances and development of multi-terminal de grids thus reducing stability issues in interconnected large power systems. Integrating these novel topologies with ac networks having different system strengths could impose challenges due to their stability constraints, which needs in depth analysis. Analysing the impact of the system strength on the performance of the converter was identified to be advantageous when adapting novel topologies to facilitate the large penetration of renewable energy generation. As an example integration of an off-shore wind farm with the mainland power network imposes a challenging situation due to the weak system strength along with the intermittent nature of the technology. A stability evaluation of the selected converter configuration under different strengths of the connected ac network was motivated through this observed requirement.

Further, the evaluation of the practical power transfer limits of a converter taking into account the constraints imposed by the configuration along with the connected network provides a better insight to its performance. The thesis has selected this as another objective, identifying shortcomings of the existing studies performed on obtaining the power transfer limits of voltage source converters with varying strengths for the ac network.

The main objectives explained above have led to the following additional objectives listed below:

1. Development of a small-signal (linear) model to evaluate the stability of the converter in the presence of small disturbances of the system variables. 
The developed small-signal model needs to be validated against a detailed, non-linear EMT simulation to verify its accuracy.

2. Utilizing the eigenvalue technique for frequency domain analysis on the statespace obtained for the converter with varying system strengths of the connected ac network. This would identify stability constraints due to insufficient damping of the oscillatory modes followed by a small perturbation in the inputs to the controllers. The stability of the converter will be further investigated by performing a sensitivity study to evaluate the impact of controller gains and measurement delays on the oscillatory stability of the converter.

3. Evaluating the practically achievable power carrying capability of the topology, constrained by the current and voltage ratings of the hybrid multilevel converter.

\subsection{Thesis Outline}

The thesis develops a linearized small-signal model of the novel hybrid multilevel converter configuration that has been proposed for HVDC transmission upon which a detailed eigenvalue analysis and a sensitivity study have been performed in order to asses the stability of the converter. The power transfer limits obtained for the restrains presented by the ratings and operational limits of the converter are evaluated for different system strengths of the connected ac system. The outline of the thesis is summarized below.

Chapter 2 presents the derivation of the linearized small-signal model of the converter. The state-space models for each subsystem have been developed and combined to obtain the overall state-space form of the converter. The model 
derived has been validated against a detailed non-linear EMT model developed on PSCAD/EMTDC through which the accuracy of the derivations have been verified. Further the derived model is modified to accommodate the variations of the dc-link voltage and this modified model has been validated against the non-linear EMT model.

Chapter 3 presents the results of the detailed eigenvalue analysis performed on the state-space model obtained for the hybrid modular multilevel converter in Chapter 2. A sensitivity study which analyses the impacts of the controller parameters including PLL gains and measurement delays of the $d q$-decoupled control scheme, on the stability of the converter have been evaluated in detail. Further, a discussion on the importance of a small-signal analysis to identify un-modelled dynamics of the converter is included to this chapter. This would at same time lead to design considerations such as selecting the appropriate transformer winding voltage ratio with the converter connected to a weak ac system.

Chapter 4 evaluates the power carrying capability of the converter connected to ac networks having different system strengths, considering the restrains imposed by the ratings and operational limits of this particular hybrid modular multilevel converter configuration.

Chapter 5 summarises the overall conclusions of the thesis and elaborates the contributions. Further, the chapter proposes future work that could be implemented using the developed small-signal model in order to improve the performance of this novel converter configuration. 


\section{Chapter 2}

\section{Modelling of the Hybrid Modular}

\section{Multilevel VSC}

A detailed analysis has to be conducted on the novel hybrid converter topology in order to evaluate its performance and stability attributes, which is a pre-requisite in controller design problems. Two approaches have been utilized in the thesis for this assessment. An Electromagnetic Transient (EMT) simulation analyses the transient response of the system using detailed non-linear models while a smallsignal analysis assesses the stability of the system for small perturbations such as an increment of the load delivered by the converter, using linearized state-space form. The former is achieved through a detailed model of one station of a point-topoint configuration of the proposed hybrid modular multilevel VSC configuration and the associated control schemes using PSCAD/EMTDC software tool. The latter is developed on MATLAB and the validation of the linearized small signal model of the converter station and controls against the detailed EMT model has been presented in this chapter. 


\subsection{Electro-magnetic Transient Modelling of the Test System}

A schematic of the test system modelled in PSCAD/EMTDC is illustrated in Fig. 2.1 with the simulation parameters given in Table 2.1, as specified in [4].

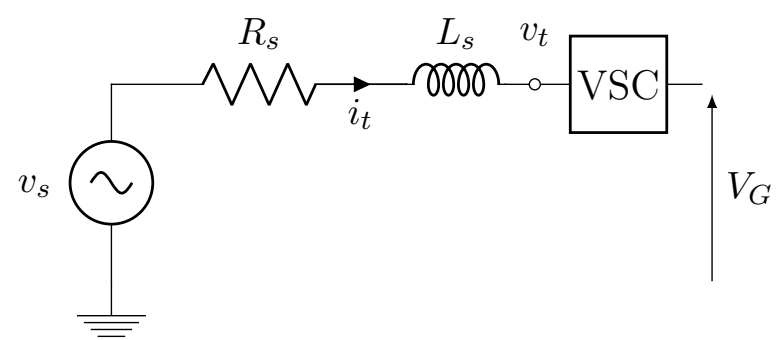

FiguRE 2.1: Schematic of the test system for the hybrid modular multilevel VSC

TABLE 2.1: Simulation Parameters [4]

\begin{tabular}{c|c}
\hline \hline Parameter & Value \\
\hline DC grid voltage & $20 \mathrm{kV}$ \\
AC grid voltage (L-L RMS) & $11 \mathrm{kV}$ \\
AC grid frequency & $50 \mathrm{~Hz}$ \\
Transformer ratio (ac side:converter side) & $1: 1.1$ \\
\hline No. of cells per phase $n_{c}$ & 9 cells \\
Cell capacitance $C_{\text {cell }}$ & $4 \mathrm{mF}$ \\
Mean cell capacitor voltage $v_{\text {cap }(r e f)}$ & $1163 \mathrm{~V}$ \\
\hline AC line inductance & $2.3 \mathrm{mH}$ \\
Line resistance & $10.37 \mathrm{~m} \Omega$ \\
DC filter inductance & $22.06 \mathrm{mH}$ \\
\hline
\end{tabular}

The MMC library available in PSCAD which includes equivalent cell components with the associated modulation techniques for multilevel schemes were incorporated in modelling this topology. The chain-link converter which contains halfbridge submodules utilizes phase-shifted Pulse Width Modulation (PWM) where these cells are switched at $100 \mathrm{~Hz}$ resulting in an effective switching frequency of 
$900 \mathrm{~Hz}$ with 9 cells per phase. The H-bridges containing series strings of IGBTs are switched at fundamental frequency at the zero voltage imposed on them.

The mean cell capacitor voltage is maintained at the value corresponding to Equation (2.1); which follows from Equation (1.6).

$$
v_{c a p(r e f)}=\frac{V_{G}}{\frac{6}{\pi} n_{c}}
$$

A $10 \mu$ s simulation time step has been used for the developed PSCAD model of the converter during this analysis.

\subsection{Development of Linearized Small-signal Model}

Small-signal stability of a given dynamic system is defined as "the ability to maintain synchronism when subjected to small disturbances". A disturbance is considered to be small if linearized equations are valid to describe the response of the system [16].

A dynamic system can be described using a set of $n$ first order, nonlinear ordinary differential equations of the form given in Equation (2.2) which is the general state-space representation of a system with $n$ number of states and $r$ number of inputs.

$$
\dot{\mathrm{X}}=f(\mathrm{X}, \mathrm{U})
$$

where

$$
\mathrm{X}=\left[x_{1}, x_{2}, \ldots, x_{n}\right]^{T} \quad \mathrm{U}=\left[u_{1}, u_{2}, \ldots, u_{r}\right]^{T} \quad f=\left[f_{1}, f_{2}, \ldots, f_{n}\right]^{T}
$$

Stability of such systems when subjected to a small disturbance could be studied 
by linearizing the set of nonlinear equations about an equilibrium point. This section provides a brief introduction to the linearization process of the above set of differential equations.

At an equilibrium point all these derivatives become simultaneously zero and the system is at steady state operating condition hence must satisfy Equation (2.3).

$$
\dot{\mathrm{X}}_{0}=f\left(\mathrm{X}_{0}, \mathrm{U}_{0}\right)=0
$$

where $\mathrm{X}_{0}$ is the state vector at the initial equilibrium point.

Applying a small perturbation from the above equilibrium point would result in the new states and inputs to be $\mathrm{X}=\mathrm{X}_{0}+\Delta \mathrm{x}$ and $\mathrm{U}=\mathrm{U}_{0}+\Delta \mathrm{u}$ respectively and substituting them in Equation (2.2) results in Equation (2.4).

$$
\dot{\mathrm{X}}_{0}+\Delta \dot{\mathrm{x}}=f\left[\left(\mathrm{X}_{0}+\Delta \mathrm{x}\right),\left(\mathrm{U}_{0}+\Delta \mathrm{u}\right)\right]
$$

Assuming the perturbation is small, the above set of equations could be linearized using Taylor series expansion and neglecting second and higher order terms; as given by Equation (2.5) - (2.6).

$$
\begin{gathered}
\dot{x}_{i 0}+\Delta \dot{x}_{i}=f_{i}\left[\left(\mathrm{X}_{0}+\Delta \mathrm{x}\right),\left(\mathrm{U}_{0}+\Delta \mathrm{u}\right)\right] \\
\dot{x}_{i 0}+\Delta \dot{x}_{i}=f_{i}\left(\mathrm{X}_{0}, \mathrm{U}_{0}\right)+\left(\frac{\partial f_{i}}{\partial x_{10}} \Delta x_{1}+\ldots+\frac{\partial f_{i}}{\partial x_{n 0}} \Delta x_{n}\right)+\left(\frac{\partial f_{i}}{\partial u_{10}} \Delta u_{1}+\ldots+\frac{\partial f_{i}}{\partial u_{r 0}} \Delta u_{r}\right)
\end{gathered}
$$

Since $\dot{x}_{i 0}=f_{i}\left(\mathrm{X}_{0}, \mathrm{U}_{0}\right)=0$ the Equation (2.6) simplifies to Equation (2.7)

$$
\Delta \dot{x}_{i}=\left(\frac{\partial f_{i}}{\partial x_{10}} \Delta x_{1}+\ldots+\frac{\partial f_{i}}{\partial x_{n 0}} \Delta x_{n}\right)+\left(\frac{\partial f_{i}}{\partial u_{10}} \Delta u_{1}+\ldots+\frac{\partial f_{i}}{\partial u_{r 0}} \Delta u_{r}\right)
$$


The partial derivatives of the above equation are evaluated at the initial equilibrium point.

Combining all linearized equations forms the state-space model of the dynamic system as in Equation (2.8).

$$
\Delta \dot{\mathrm{X}}=A \Delta \mathrm{X}+B \Delta \mathrm{U}
$$

in which,

$$
\begin{aligned}
\Delta \mathrm{X} & =\left[\Delta x_{1}, \Delta x_{2}, \ldots, \Delta x_{n}\right] \\
\Delta \mathrm{U} & =\left[\Delta u_{1}, \Delta u_{2}, \ldots, \Delta u_{r}\right] \\
\mathrm{A} & =\left[\begin{array}{lll}
\frac{\partial f_{1}}{\partial x_{10}} & \cdots & \frac{\partial f_{1}}{\partial x_{n 0}} \\
\ldots & \ldots & \ldots \\
\frac{\partial f_{n}}{\partial x_{10}} & \cdots & \frac{\partial f_{n}}{\partial x_{n 0}}
\end{array}\right] \\
\mathrm{B} & =\left[\begin{array}{lll}
\frac{\partial f_{1}}{\partial u_{10}} & \ldots & \frac{\partial f_{1}}{\partial u_{r 0}} \\
\ldots & \ldots & \ldots \\
\frac{\partial f_{n}}{\partial u_{10}} & \cdots & \frac{\partial f_{n}}{\partial u_{r 0}}
\end{array}\right]
\end{aligned}
$$

The small-signal model for one station of the point-to-point hybrid modular multilevel converter is obtained using the above linearization process which is explained in Sections (2.2.1)-(2.2.6).

\subsubsection{AC System Model}

The ac network connected to the converter station is represented by a Thevenin equivalent circuit that comprises of a voltage source and a series RL impedance, connected to an ideal transformer as shown in Fig. 2.1. Applying Kirchoff's voltage 
law to the circuit shown, Equation (2.9) could be obtained.

$$
v_{s}-v_{t}=L_{s} \frac{d i_{t}}{d t}+R_{s} i_{t}
$$

In small-signal stability assessment of system instabilities due to low-frequency electromechanical oscillations, the ac network which includes transmission lines and series, shunt compensation devices are represented by the admittance matrix which could capture frequencies accurately only within the range of 0.1-2 $\mathrm{Hz}$. However, small-signal instabilities in systems incorporating control interactions with fast acting devices such as HVDC schemes, the Sub-Synchronous Oscillations (SSO) are observed in the range of $5-55 \mathrm{~Hz}$. Thus for accurate representation of the ac network a dynamic model needs to be incorporated [17].

This thesis hence represents the ac network connected to the converter using a dynamic network model which enables to incorporate the dynamic relationship of the RLC components in the network with the phasor quantities of voltages and currents. Substituting complex rotating phasor relationship of currents and voltages to Equation (2.9) results in Equation (2.10) which is simplified to obtain the phasor relationship given in Equation (2.11) [18].

$$
\begin{aligned}
& \left(V_{s R}+j V_{s I}\right) e^{j \omega_{0} t}-\left(V_{t R}+j V_{t I}\right) e^{j \omega_{0} t}=L_{s} \frac{d\left(I_{t R}+j I_{t I}\right) e^{j \omega_{0} t}}{d t}+R_{s}\left(I_{t R}+j I_{t I}\right) e^{j \omega_{0} t} \\
& \left(V_{s R}+j V_{s I}\right)-\left(V_{t R}+j V_{t I}\right)=L_{s} \frac{d\left(I_{t R}+j I_{t I}\right)}{d t}+\left(R_{s}+j \omega_{0} L_{s}\right)\left(I_{t R}+j I_{t I}\right)
\end{aligned}
$$

Hence the linearized state space model of the AC system in per unit terms is given by Equation (2.12).

$$
\Delta \dot{\mathrm{X}}_{a c}=A_{a c} \Delta \mathrm{X}_{a c}+B_{a c} \Delta \mathrm{U}_{a c}+E_{a c} \Delta \mathrm{V}_{t}
$$


where

$$
\begin{aligned}
\Delta \mathrm{X}_{a c}= & {\left[\Delta I_{t R}, \Delta I_{t I}\right]^{T} } \\
\Delta \mathrm{U}_{a c}= & {\left[\Delta V_{s R}, \Delta V_{s I}\right]^{T} } \\
\Delta \mathrm{V}_{t}= & {\left[\Delta V_{t R}, \Delta V_{t I}\right]^{T} } \\
A_{a c}= & {\left[\begin{array}{cc}
\frac{-R_{s} \omega_{0}}{L_{s}} & \omega_{0} \\
-\omega_{0} & \frac{-R_{s} \omega_{0}}{L_{s}}
\end{array}\right] } \\
B_{a c}= & {\left[\begin{array}{cc}
\frac{\omega_{0}}{L_{s}} & 0 \\
0 & \frac{\omega_{0}}{L_{s}}
\end{array}\right] } \\
E_{a c}= & {\left[\begin{array}{cc}
\frac{-\omega_{0}}{L_{s}} & 0 \\
0 & \frac{-\omega_{0}}{L_{s}}
\end{array}\right] }
\end{aligned}
$$

\subsubsection{Phase-Locked Loop Model}

The control system of the converter requires accurately measured phase angle of the ac bus voltage to produce voltage orders that are subsequently converted to gate pulse signals to the submodules of the chain-link converter as well as the Hbridges. Phase-locked loop is the device which is used in power system applications to measure the phase angle of the reference bus voltage. Among different PLL mechanisms incorporated under the current context, the Synchronous Reference Frame PLL (SRF-PLL) [18] is the most commonly used configuration in power system applications for control design purposes and the control block diagram is illustrated in Fig.2.2.

The PLL provides an estimate for the phase angle $\left(\delta_{m}\right)$ which is then compared against the actual angle $(\delta)$. With per-unitized voltages, the error signal between the estimated and the actual angles could be expressed as $\sin \left(\delta-\delta_{m}\right)$, which is passed across a PI controller to obtain the angular frequency error. The nominal 
angular frequency $\omega_{0}$ is added to this frequency error and by integrating the resultant value provides an estimation for the phase angle $\left(\delta_{m}\right)$. This estimated phase angle is then utilized to convert the voltages and currents which are modelled in the $R I$ domain to the synchronous $d q$ frame and incorporated in the decoupled control scheme of the converter. The conversion of converter quantities between these frames and the control circuitry are explained in sections 2.2.4 and 2.2.5.1 respectively.

The actual phase angle $(\delta)$ is obtained from the real and imaginary components of the reference ac voltage $v_{s}$ which is $\tan ^{-1}\left(\frac{V_{s I}}{V_{s R}}\right)$

The governing dynamic equations of the SRF-PLL in per unit terms are given by Equation (2.13) and (2.14).

$$
\begin{gathered}
\dot{x}_{p l l}=\sin \left(\delta-\delta_{m}\right) \\
\dot{\delta}_{m}=K_{P p l l} \sin \left(\delta-\delta_{m}\right)+K_{\text {Ipll }} x_{p l l}+\omega_{0}
\end{gathered}
$$

The state-space model of the PLL is thus obtained from Equation (2.15).

$$
\Delta \dot{\mathrm{X}}_{p l l}=A_{p l l} \Delta \mathrm{X}_{p l l}+E_{p l l} \Delta \mathrm{V}_{s}
$$

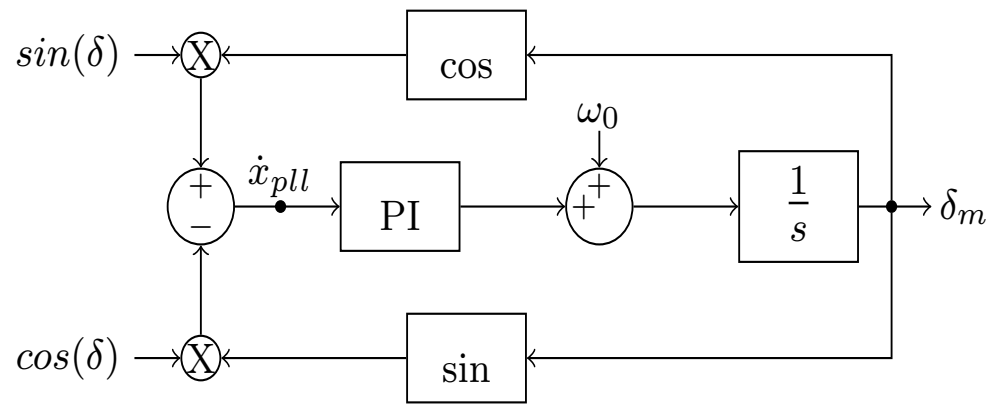

FiguRE 2.2: PLL block diagram 


$$
Y_{\alpha} \rightarrow \frac{1}{1+s T_{\alpha}} \rightarrow Y_{\alpha(m)}
$$

Figure 2.3: Measurement filter block

where,

$$
\begin{aligned}
\Delta \mathrm{X}_{p l l}= & {\left[\Delta x_{p l l}, \Delta \delta_{m}\right]^{T} } \\
\Delta \mathrm{V}_{s}= & {\left[\Delta V_{s R}, \Delta V_{s I}\right]^{T} } \\
A_{p l l}= & {\left[\begin{array}{cc}
0 & -1 \\
K_{\text {Ipll }} & -K_{P p l l}
\end{array}\right] } \\
E_{p l l}= & {\left[\begin{array}{cc}
\frac{-V_{s I 0}}{V_{s 0}^{2}} & \frac{V_{s R 0}}{V_{s 0}^{2}} \\
-K_{P p l l} \frac{V_{s I 0}}{V_{s 0}^{2}} & K_{P p l l} \frac{V_{s R 0}}{V_{s 0}^{2}}
\end{array}\right] }
\end{aligned}
$$

\subsubsection{Measurement Filter Model}

The outer-loop control of the de-coupled control scheme of the converter uses the measured active and reactive power quantities as inputs to generate the reference currents for the inner-loop control. The measurement transducers incorporate filters to remove noise from the measured signal in order to improve its accuracy. In EMT simulations this has been achieved by adjusting a smoothing time constant in the measuring device which introduces a delay. Thus the delay incorporated with these measurement filters has been accounted in the small-signal analysis as explained below.

A first order filter model has been used for this purpose which is shown in Fig. 2.3 and the dynamic equation associated with the above measurement filter model is given in Equation (2.16). 


$$
\dot{Y}_{\alpha(m)}=\frac{1}{T_{\alpha}}\left(Y_{\alpha}-Y_{\alpha(m)}\right)
$$

Thus the dynamics associated with the measurement of active and reactive power supplied by the converter are given by Equation (2.17) and (2.18) respectively.

$$
\begin{gathered}
\dot{P}_{(m)}=\frac{1}{T_{(p m)}}\left(P_{s}-P_{(m)}\right) \\
\dot{Q}_{(m)}=\frac{1}{T_{(q m)}}\left(Q_{s}-Q_{(m)}\right)
\end{gathered}
$$

Substituting $P_{s}$ and $Q_{s}$ in terms of $R I$ frame voltage and current quantities $\left(V_{s R}, V_{s I}, I_{t R}, I_{t I}\right)$ and linearizing, the state-space model of the measurement filter delays could be obtained as shown in Equation (2.19).

$$
\Delta \dot{\mathrm{X}}_{m}=A_{m} \Delta \mathrm{X}_{m}+E_{m} \Delta \mathrm{V}_{s}+F_{m} \Delta \mathrm{I}_{t}
$$

where,

$$
\begin{aligned}
\Delta \mathrm{X}_{m}= & {\left[\Delta P_{(m)}, \Delta Q_{(m)}\right]^{T} } \\
\Delta \mathrm{V}_{s}= & {\left[\Delta V_{s R}, \Delta V_{s I}\right]^{T} } \\
\Delta \mathrm{I}_{t}= & {\left[\Delta I_{t R}, \Delta I_{t I}\right]^{T} } \\
A_{m}= & {\left[\begin{array}{cc}
-\frac{1}{T_{p m}} & 0 \\
0 & -\frac{1}{T_{q m}}
\end{array}\right] } \\
E_{m}= & {\left[\begin{array}{cc}
\frac{V_{s R 0}}{T_{p m}} & \frac{V_{s I 0}}{T_{p m}} \\
\frac{V_{s I 0}}{T_{q m}} & -\frac{V_{s R 0}}{T_{q m}}
\end{array}\right] } \\
F_{m}= & {\left[\begin{array}{cc}
\frac{I_{t R 0}}{T_{p m}} & \frac{I_{t I 0}}{T_{p m}} \\
-\frac{I_{t I 0}}{T_{q m}} & \frac{I_{t R 0}}{T_{q m}}
\end{array}\right] }
\end{aligned}
$$




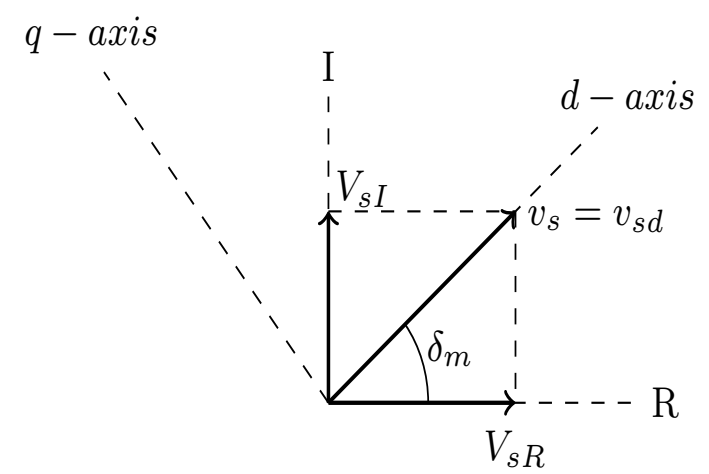

FiguRE 2.4: RI- $d q$ transformation

\subsubsection{Axis Transformation}

The converter control system requires synchronous reference frame $d q$ current and voltage components as inputs where as the ac system components are modelled using the standard $R I$ co-ordinate system. Thus transformations from $R I$ frame to $d q$ frame and vice-versa are required to combine the network and control variables of the state-space system. This transformation could be achieved as illustrated in Fig. 2.4. The phase angle $\left(\delta_{m}\right)$ required for this transformation is obtained from the PLL output as explained in section 2.2.2.

The $R I$ to $d q$ transformation and the inverse transformation matrices are as given in Equation (2.20) and (2.21).

$$
\begin{aligned}
& {\left[\begin{array}{l}
x_{d} \\
x_{q}
\end{array}\right]=\left[\begin{array}{cc}
\cos \delta_{m} & \sin \delta_{m} \\
-\sin \delta_{m} & \cos \delta_{m}
\end{array}\right]\left[\begin{array}{l}
x_{R} \\
x_{I}
\end{array}\right]} \\
& {\left[\begin{array}{l}
x_{R} \\
x_{I}
\end{array}\right]=\left[\begin{array}{ll}
\cos \delta_{m} & -\sin \delta_{m} \\
\sin \delta_{m} & \cos \delta_{m}
\end{array}\right]\left[\begin{array}{l}
x_{d} \\
x_{q}
\end{array}\right]}
\end{aligned}
$$

Hence the linearized algebraic equations for $R I$ to $d q$ frame transformation of voltage and current components and vice versa could be obtained from Equations 
(2.22)-(2.24)

$$
\begin{gathered}
\Delta \mathrm{V}_{s}^{d q}=K_{v} \Delta \mathrm{V}_{s}+M_{v} \Delta \delta_{m} \\
\Delta \mathrm{I}_{t}^{d q}=K_{i} \Delta \mathrm{I}_{t}+M_{i} \Delta \delta_{m} \\
\Delta \mathrm{V}_{t}=J_{v} \Delta \mathrm{V}_{t}^{d q}+N_{v} \Delta \delta_{m}
\end{gathered}
$$

where,

$$
\begin{aligned}
& \Delta \mathrm{V}_{s}^{d q}=\left[\Delta v_{s d}, \Delta v_{s q}\right]^{T} \\
& \Delta \mathrm{V}_{s}=\left[\Delta V_{s R}, \Delta V_{s I}\right]^{T} \\
& \Delta \mathrm{I}_{t}^{d q}=\left[\Delta i_{t d}, \Delta i_{t q}\right]^{T} \\
& \Delta \mathrm{I}_{t}=\left[\Delta I_{t R}, \Delta I_{t I}\right]^{T} \\
& \Delta \mathrm{V}_{t}=\left[\Delta V_{t R}, \Delta V_{t I}\right]^{T} \\
& \Delta \mathrm{V}_{t}^{d q}=\left[\Delta v_{t d}, \Delta v_{t q}\right]^{T} \\
& K_{v}=\left[\begin{array}{cc}
\cos \delta_{m 0} & \sin \delta_{m 0} \\
-\sin \delta_{m 0} & \cos \delta_{m 0}
\end{array}\right] \\
& K_{i}=\left[\begin{array}{cc}
\cos \delta_{m 0} & \sin \delta_{m 0} \\
-\sin \delta_{m 0} & \cos \delta_{m 0}
\end{array}\right] \\
& J_{v}=\left[\begin{array}{cc}
\cos \delta_{m 0} & -\sin \delta_{m 0} \\
\sin \delta_{m 0} & \cos \delta_{m 0}
\end{array}\right] \\
& M_{v}=\left[\begin{array}{l}
-V_{s R 0} \sin \delta_{m 0}+V_{s I 0} \cos \delta_{m 0} \\
-V_{s R 0} \cos \delta_{m 0}-V_{s I 0} \sin \delta_{m 0}
\end{array}\right] \\
& M_{i}=\left[\begin{array}{l}
-I_{t R 0} \sin \delta_{m 0}+I_{t I 0} \cos \delta_{m 0} \\
-I_{t R 0} \cos \delta_{m 0}-I_{t I 0} \sin \delta_{m 0}
\end{array}\right] \\
& N_{v}=\left[\begin{array}{c}
-V_{t d 0} \sin \delta_{m 0}-V_{t q 0} \cos \delta_{m 0} \\
V_{t d 0} \cos \delta_{m 0}-V_{t q 0} \sin \delta_{m 0}
\end{array}\right]
\end{aligned}
$$




\subsubsection{Control System Model}

Control system for the hybrid modular multilevel VSC consists of an ac side control circuitry as well as a dc side control scheme. The ac side controller adjusts the active and reactive power exchanged with the grid according to the input demands, which is achieved through a rotating reference frame $d q$ positive sequence line current controller as shown in Fig 2.5. This standard arrangement, also referred to as vector current control is based on independent control of active and reactive power by synchronous reference frame $d q$ current components and consists of two control loops with cascaded PI controllers namely the outer-loop and inner-loop controller [19].

The slower outer-loop controller uses the desired active and reactive power demands $P_{(r e f)}, Q_{(r e f)}$ with the measured power quantities $P_{(m)}, Q_{(m)}$ to generate the references for the $d$ and $q$-axis current components $i_{t d(r e f)}, i_{t q(r e f)}$ respectively. As shown in Fig. 2.5 active power is selected as the control variable to produce the

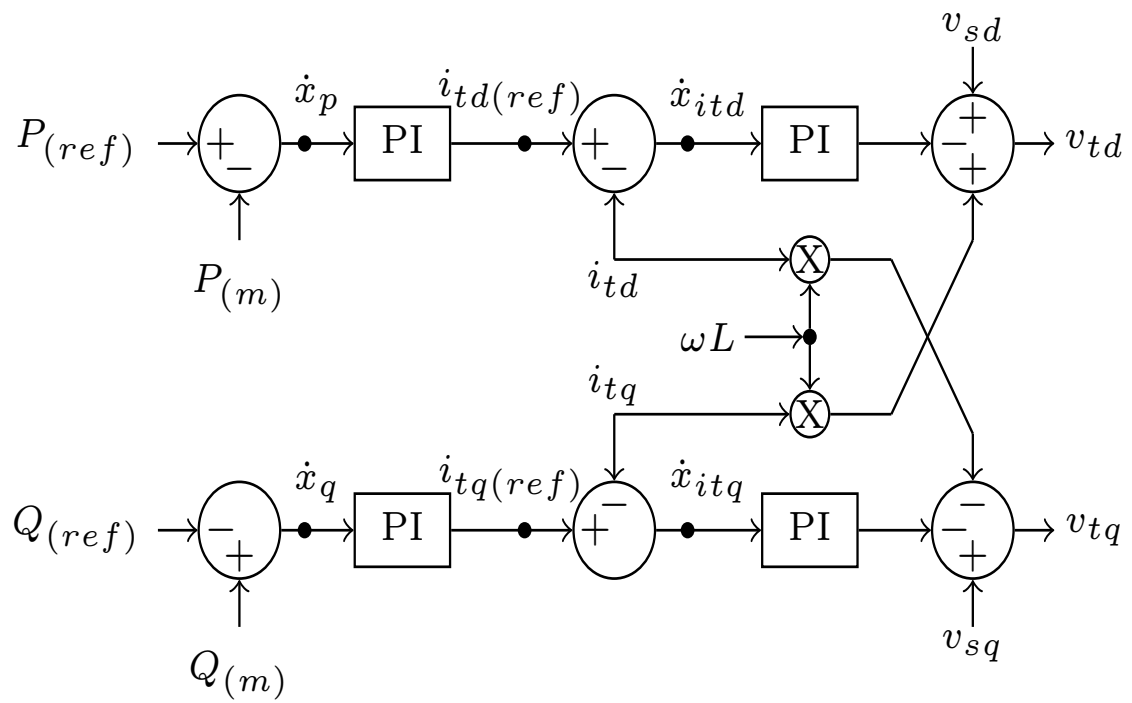

Figure 2.5: Decoupled $d q$ control scheme; redrawn with modifications from Fig.10 in [4] 
d-axis current reference and reactive power for the q-axis current reference. The error between the desired and the measured quantities, regulated by a suitably tuned PI controller produces these $d q$-frame reference current components.

The faster inner-loop controller uses these references generated by the outer-loop to regulate the measured dq-currents of the converter to meet the desired values. A PI controller arrangement then generates the voltage orders $v_{t d}, v_{t q}$, combining the output of these PI controllers with the measured $d q$-axis voltage components of the reference ac bus voltage and de-coupling terms. The development of the small-signal model for the ac control scheme of the converter is explained in 2.2.5.1.

The dc control scheme of the converter regulates the power balance between ac and dc sides thereby generating the reference for the dc-link current. This desired current reference is utilized in order to generate the demand for the voltage to be applied across the cascaded chain-link converters of the three phases, thereby obtaining the triplen harmonic compensation required to maintain a constant voltage at the dc-link. The above control scheme has been explained with further details in 2.2.5.2.

\subsubsection{Small-signal Model of the AC Control System}

Model of a PI controller used in the above control scheme is given in Fig. 2.6 whereas the dynamic and linear equations associated with that are given by Equation (2.25) and (2.26) respectively.

$$
\begin{gathered}
\dot{X}_{\alpha}=\left(Y_{\alpha(r e f)}-Y_{\alpha(m)}\right) \\
Z=K_{P \alpha}\left(Y_{\alpha(r e f)}-Y_{\alpha(m)}\right)+K_{I \alpha} X_{\alpha}
\end{gathered}
$$




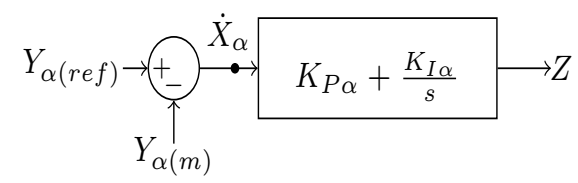

FiguRE 2.6: PI controller block

Thus the state equations of the outer-loop controller for the hybrid VSC could be expressed as given in Equations (2.27) and (2.28).

$$
\begin{gathered}
\dot{x}_{P}=\left(P_{(r e f)}-P_{(m)}\right) \\
\dot{x}_{Q}=\left(Q_{(m)}-Q_{(r e f)}\right)
\end{gathered}
$$

The outputs from the PI controllers of the outer-loop control are the reference $d$ and $q$ axis currents as given in Equations (2.29) and (2.30) respectively.

$$
\begin{aligned}
& i_{t d(r e f)}=K_{P p}\left(P_{(r e f)}-P_{(m)}\right)+K_{I p} x_{P} \\
& i_{t q(r e f)}=K_{P q}\left(Q_{(m)}-Q_{(r e f)}\right)+K_{I q} x_{Q}
\end{aligned}
$$

The state equations for the inner-loop control could be obtained using these current references as given in Equation (2.31) and (2.32)

$$
\begin{aligned}
& \dot{x}_{i t d}=\left(i_{t d(r e f)}-i_{t d}\right) \\
& \dot{x}_{i t q}=\left(i_{t q(r e f)}-i_{t q}\right)
\end{aligned}
$$

The reference currents in the above equation are substituted from Equations (2.29) and (2.30).

The per-unitized state-space model of the ac control system for the converter is obtained by linearizing Equations (2.27), (2.28), (2.31) and (2.32) and combining 
them into a state-space form as given in Equation (2.33).

$$
\Delta \dot{\mathrm{X}}_{C a c}=A_{C a c} \Delta \mathrm{X}_{C a c}+A_{C a c}^{*} \Delta \mathrm{X}_{m}+B_{C a c} \Delta \mathrm{U}_{C a c}+F_{C a c} \Delta \mathrm{I}_{t}^{d q}
$$

where,

$$
\begin{aligned}
& \Delta \mathrm{X}_{C a c}=\left[\Delta x_{P}, \Delta x_{Q}, \Delta x_{i t d}, \Delta x_{i t q}\right]^{T} \\
& \Delta \mathrm{X}_{m}=\left[\Delta P_{(m)}, \Delta Q_{(m)}\right]^{T} \\
& \Delta \mathrm{U}_{C a c}=\left[\Delta P_{(r e f)}, \Delta Q_{(r e f)}\right]^{T} \\
& \Delta \mathrm{I}_{t}^{d q}=\left[\Delta i_{t d}, \Delta i_{t q}\right]^{T} \\
& A_{C a c}=\left[\begin{array}{cccc}
0 & 0 & 0 & 0 \\
0 & 0 & 0 & 0 \\
k_{1} K_{I p} & 0 & 0 & 0 \\
0 & k_{1} K_{I q} & 0 & 0
\end{array}\right] \\
& A_{C a c}^{*}=\left[\begin{array}{cc}
-1 & 0 \\
0 & 1 \\
k_{1} K_{P p} & 0 \\
0 & k_{1} K_{P q}
\end{array}\right] \\
& B_{C a c}=\left[\begin{array}{cc}
1 & 0 \\
0 & -1 \\
k_{1} K_{P p} & 0 \\
0 & -k_{1} K_{P q}
\end{array}\right]
\end{aligned}
$$




$$
\begin{aligned}
F_{C a c} & =\left[\begin{array}{cc}
0 & 0 \\
0 & 0 \\
-1 & 0 \\
0 & -1
\end{array}\right] \\
k_{1} & =\frac{M V A_{b a s e}}{I_{\text {base }} * 1000}
\end{aligned}
$$

The EMT simulation model of the converter was developed on the basis of actual units whereas the small-signal model is developed on a per unit basis. Thus the PI controller gains $\left(K_{P \alpha}, K_{I \alpha}\right)$ used in the EMT simulation had to be scaled in order to be used with the equivalent small-signal model and these scaling factors are denoted in this thesis by $k_{i}$.

The output signals from the inner-loop control are combined with the $d q$ components of the ac bus voltage and de-coupling terms in order to obtain the voltage order signals $v_{t d}$ and $v_{t q}$. These will produce the gate pulses to the half-bridge submodules of the wave-shaping circuit and for the H-bridge IGBT strings (director switches) in the main power circuit.

The voltage orders for the converter are given by Equation (2.34).

$$
\Delta \mathrm{V}_{t}^{d q}=C_{o} \Delta \mathrm{X}_{C a c}+C_{o}^{*} \Delta \mathrm{X}_{m}+D_{o} \Delta \mathrm{U}_{C a c}+K_{o} \Delta \mathrm{V}_{s}^{d q}++M_{o} \Delta \mathrm{I}_{t}^{d q}
$$

where,

$$
\begin{aligned}
\Delta \mathrm{X}_{C a c} & =\left[\Delta x_{P}, \Delta x_{Q}, \Delta x_{i t d}, \Delta x_{i t q}\right]^{T} \\
\Delta \mathrm{X}_{m} & =\left[\Delta P_{(m)}, \Delta Q_{(m)}\right]^{T}
\end{aligned}
$$




$$
\begin{aligned}
& \Delta \mathrm{U}_{C a c}=\left[\Delta P_{(r e f)}, \Delta Q_{(r e f)}\right]^{T} \\
& \Delta \mathrm{V}_{s}^{d q}=\left[\Delta v_{s d}, \Delta v_{s q}\right]^{T} \\
& \Delta \mathrm{V}_{t}^{d q}=\left[\Delta v_{t d}, \Delta v_{t q}\right]^{T} \\
& \Delta \mathrm{I}_{t}^{d q}=\left[\Delta i_{t d}, \Delta i_{t q}\right]^{T} \\
& C_{o}=\left[\begin{array}{cccc}
-k_{1} k_{2} K_{P i d} K_{I p} & 0 & -k_{2} K_{\text {Iid }} & 0 \\
0 & -k_{1} k_{2} K_{P i q} K_{I q} & 0 & -k_{2} K_{I i q}
\end{array}\right] \\
& C_{o}^{*}=\left[\begin{array}{cc}
k_{1} k_{2} K_{P i d} K_{P p} & 0 \\
0 & -k_{1} k_{2} K_{P i q} K_{P q}
\end{array}\right] \\
& K_{o}=\left[\begin{array}{ll}
1 & 0 \\
0 & 1
\end{array}\right] \\
& M_{o}=\left[\begin{array}{cc}
k_{2} K_{P i d} & \omega L \\
-\omega L & k_{2} K_{P i q}
\end{array}\right] \\
& k_{2}=\frac{i_{\text {base }}}{e_{\text {base }}}
\end{aligned}
$$

\subsubsection{Small-signal Model of the DC Control System}

In the arrangement of the hybrid modular multilevel VSC, the wave-shaping circuit which consists of a chain-link of half-bridge submodules for the three phases are connected in series at the dc terminals of the H-bridges. Thus the voltage across the dc link is created by the summation of rectified ac voltages repeating twice per cycle, that are synthesized by these chain-link converters to fulfil the requirements of the ac network. Hence the converter voltage magnitude cannot be varied without affecting its dc-link voltage which results in a fixed modulation index for the converter as explained in 1.3.4.2. This inhibits the capability of reactive power compensation at the presence of fixed dc voltage. In order to be 
capable of regulating the converter voltage while maintaining a constant dc voltage, this converter configuration utilizes the technique which introduces a small amount of zero phase sequence $3^{\text {rd }}$ harmonic voltage. Thus the voltage synthesized by the wave-shaping converters will be the addition of the fundamental and the triplen harmonic, which further has the advantage of not being seen by the ac network [4]. This provides a direct component of voltage over a half cycle that could be controlled either positively or negatively to compensate for the changes in the rectified ac voltage reference hence restore the dc link voltage to its nominal value.

This configuration requires to have two additional control loops for the dc side capacitor voltages and dc-link current of the hybrid converter which are shown in Fig 2.7 and Fig. 2.8 respectively. The cascaded PI controller arrangement consists of a slower outer-loop control that regulates the square of the cell capacitor voltages which is proportional to the total power to the chain-link converter. This enables to maintain the balance between the dc side power and ac side active power while producing a reference for the dc-link current $\left(i_{d c(r e f)}\right)$. The faster inner-loop control utilizes the error between this reference and the actual dc-link current $\left(i_{d c}\right)$ through a PI controller to regulate the voltage to be applied across the dc-link $\bar{v}_{d c}$, by which the magnitude of the required $3^{\text {rd }}$ harmonic voltage compensation $\alpha_{3}$ is calculated as explained in 1.3.4.2.

As illustrated in Fig. 2.7 the first two state equations of the dc control system are developed for the slower outer-loop control. A value that is proportional to the chain-link power has been computed by the squared sum of cell-voltage reference $v_{c a p(r e f)}$. This will be compared with the squared sum of the measured capacitor voltages which would be proportional to the actual power consumed by the chainlink. The error between the two will be given to a PI-controller whose output is the desired chain-link power $P_{c l(r e f)}$ which will also provide an estimate of the 


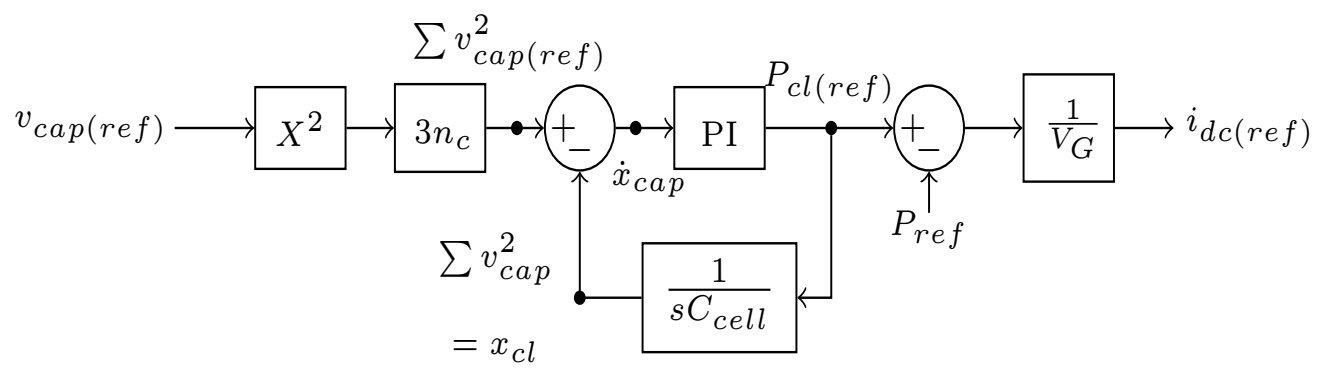

Figure 2.7: Capacitor voltage control scheme (outer-loop); redrawn from Fig. 11 of $[4]^{1}$

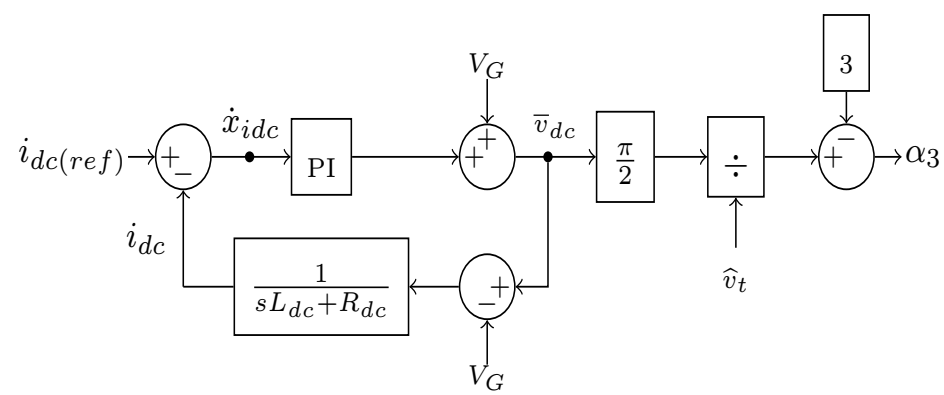

FiguRE 2.8: DC link current control scheme (inner-loop); redrawn from Fig. 12 of $[4]^{1}$

power balance between the ac and the dc sides. The governing dynamic equations are given by Equation (2.35) and (2.36).

$$
\begin{gathered}
\dot{x}_{c a p}=3 n_{c} v_{c a p(r e f)}^{2}-x_{c l} \\
\dot{x}_{c l}=\frac{1}{C_{c e l l}}\left(K_{P c l}\left(3 n_{c} v_{c a p(r e f)}^{2}-x_{c l}\right)+K_{I c l} x_{c a p}\right)
\end{gathered}
$$

The output of the PI controller is then feed forwarded with the ac power demand $\left(P_{r e f}\right)$ which results in the dc power demand. Assuming a steady state mean composite chain-link voltage $V_{G}$ across the cells, the reference dc-link current $i_{d c(r e f)}$

\footnotetext{
${ }^{1}$ C) 2013 IEEE
} 
can be calculated as given in Equation (2.37).

$$
i_{d c(r e f)}=\frac{1}{V_{G}}\left(k_{3} K_{P c l}\left(3 n_{c} v_{c a p(r e f)}^{2}-x_{c l}\right)+k_{3} K_{\text {Icl }} x_{c a p}-P_{(r e f)}\right)
$$

where $k_{3}=\frac{V_{d c, \text { base }}^{2}}{M V A_{\text {base }}}$

The remaining dynamic equation is obtained from the inner-loop control of the dc control scheme as given in Equation (2.38).

$$
\dot{x}_{i d c}=i_{d c(r e f)}-i_{d c}
$$

$i_{d c(r e f)}$ in the above equation can be substituted from Equation (2.37). Linearizing Equation (2.35), (2.36) and (2.38) the overall state space model for the dc side controls can be obtained as given in Equation (2.39).

$$
\Delta \dot{\mathrm{X}}_{C d c}=A_{C d c} \Delta \mathrm{X}_{C d c}+A_{C d c}^{*} \Delta i_{d c}+B_{C d c} \Delta \mathrm{U}_{C d c}
$$

where,

$$
\begin{aligned}
\Delta \mathrm{X}_{C d c} & =\left[\Delta x_{c a p}, \Delta x_{c l}, \Delta x_{i d c}\right]^{T} \\
\Delta \mathrm{U}_{C d c} & =\Delta P_{(r e f)} \\
A_{C d c} & =\left[\begin{array}{ccc}
0 & -1 & 0 \\
\frac{K_{I c l}}{C_{c e l l}} & -\frac{K_{P c l}}{C_{c e l l}} & 0 \\
\frac{k_{3} K_{I c l}}{V_{G}} & -\frac{k_{3} K_{P c l}}{V_{G}} & 0
\end{array}\right] \\
A_{C d c}^{*} & =-1 \\
B_{C d c} & =\left[\begin{array}{c}
0 \\
0 \\
-\frac{1}{V_{G}}
\end{array}\right]
\end{aligned}
$$




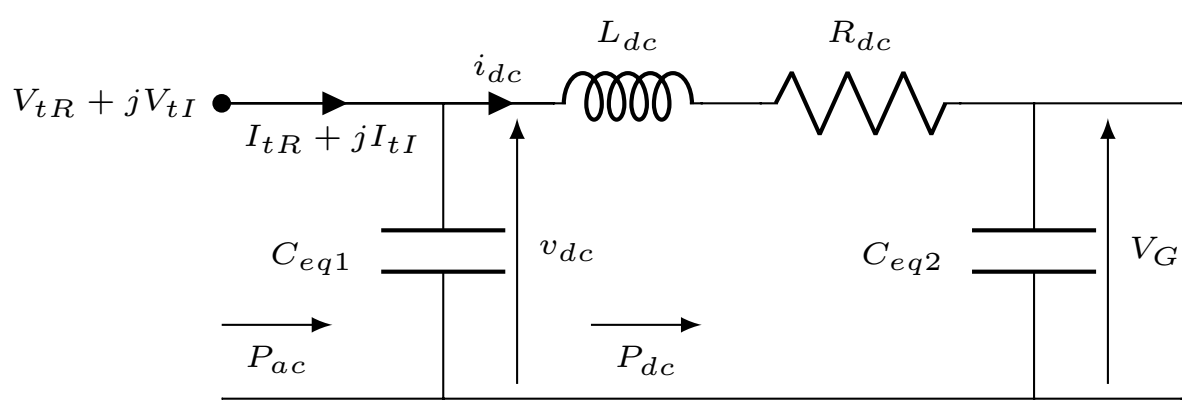

Figure 2.9: Equivalent circuit of the DC system

\subsubsection{System Model}

The DC system of the converter is modelled as a transmission line having a smoothing inductance of $L_{d c}$ and a line resistance of $R_{d c}$ connecting to the converter station at the other end. The voltage at this end is maintained constant at $V_{G}$ by the controls of this converter station.

The dynamics of the submodule capacitors need to be included in the small-signal model hence the equivalent circuit representation of the dc-link of the hybrid converter is obtained as illustrated in Fig. 2.9. $C_{e q 1}$ models the equivalent capacitance of the submodule capacitors that are inserted to generate the voltage $\bar{v}_{d c}$ which is applied across the dc-link as demanded from the inner-loop control, explained in 2.2.5.2. $C_{e q 2}$ is a representation of the submodules of the chain-link converter in the opposite end station and are not modelled in the small-signal model of the one-converter test system.

The equation which describes the dynamics of the submodule capacitors could be obtained by considering the power balance between the ac and the dc sides as given in Equation (2.40).

$$
\frac{d\left(\frac{1}{2} C_{e q} v_{d c}^{2}\right)}{d t}=P_{a c}-P_{d c}-P_{l o s s}
$$


where

$$
\begin{aligned}
P_{a c} & =V_{t R} I_{t R}+V_{t I} I_{t I} \\
P_{d c} & =v_{d c} i_{d c} \\
C_{e q} & =\frac{C_{c e l l}}{\left(\frac{\bar{v}_{d c}}{\left.v_{c a p(r e f)}\right)}\right)}
\end{aligned}
$$

$P_{\text {loss }}$ is assumed to be negligible since the H-bridge converters are soft switched while the chain-link converter which utilizes multilevel modulation schemes involving high frequency switching is outside the main conduction path, accounting for low converter losses due to switching [4]. Substituting for $P_{a c}, P_{d c}$ and $C_{e q}$ in Equation (2.40), the equation governing dynamics of the submodules could be obtained.

Equation (2.41) in per unit terms describes the dynamics of the dc transmission line connecting with the converter station at the other end.

$$
v_{d c}-V_{G}=L_{d c} \frac{d i_{d c}}{d t}+R_{d c} i_{d c}
$$

Linearizing Equations (2.40) and (2.41), the state-space model of the DC system of the hybrid converter could be obtained as given in Equation (2.42).

$$
\Delta \dot{\mathrm{X}}_{d c}=A_{d c} \Delta \mathrm{X}_{d c}+A_{d c}^{*} \Delta \mathrm{X}_{C d c}+B_{d c} \Delta \mathrm{U}_{d c}+E_{d c} \Delta \mathrm{V}_{t}+F_{d c} \Delta \mathrm{I}_{t}
$$


where

$$
\begin{aligned}
& \Delta \mathrm{X}_{d c}=\left[\Delta v_{d c}, \Delta i_{d c}\right] \\
& \Delta \mathrm{X}_{C d c}=\left[\Delta x_{c a p}, \Delta x_{c l}, \Delta x_{i d c}\right] \\
& \Delta U_{d c}=\Delta P_{r e f} \\
& \Delta \mathrm{V}_{t}=\left[\Delta V_{t R}, \Delta V_{t I}\right] \\
& \Delta \mathrm{I}_{t}=\left[\Delta I_{t R}, \Delta I_{t I}\right] \\
& A_{d c}=\left[\begin{array}{cc}
\frac{\left(m_{10}-m_{2}\right)}{m_{1}} & \frac{\left(m_{11}+m_{14}+m_{6}\right)}{m_{1}} \\
\frac{\omega_{0}}{L_{d c}} & \frac{-\omega_{0} R_{d c}}{L_{d c}}
\end{array}\right] \\
& A_{d c}^{*}=\left[\begin{array}{ccc}
\frac{\left(m_{13}-m_{4}\right)}{m_{1}} & \frac{\left(m_{12}+m_{3}\right)}{m_{1}} & \frac{\left(m_{15}-m_{7}\right)}{m_{1}} \\
0 & 0 & 0
\end{array}\right] \\
& B_{d c}=\left[\begin{array}{c}
\frac{\left(m_{16}+m_{5}\right)}{m_{1}} \\
0
\end{array}\right] \\
& E_{d c}=\left[\begin{array}{cc}
\frac{m_{17}}{m_{1}} & \frac{m_{18}}{m_{1}} \\
0 & 0
\end{array}\right] \\
& F_{d c}=\left[\begin{array}{cc}
\frac{m_{8}}{m_{1}} & \frac{m_{9}}{m_{1}} \\
0 & 0
\end{array}\right]
\end{aligned}
$$

Derivation of the coefficients of the DC system state space model is explained in A.

\subsubsection{Overall State-Space Model of the Hybrid Modular Multilevel Converter}

Combination of all state-space forms obtained in the above sections for each component of the converter, the overall state-space model of the converter could be 
formulated which could be expressed in a generalized form as given in Equation $(2.43)$.

$$
\Delta \dot{\mathrm{X}}=A \Delta \mathrm{X}+B \Delta \mathrm{U}
$$

MATLAB software tool was utilized in order to combine the state-space models for the ac system, PLL, measurement filters, ac and dc control circuitry and the dc system hence obtain the linearized overall state-space model of the converter which is intended to be used for stability analysis as explained in the next chapters of this thesis.

The overall model consists of 15 state variables and 4 control inputs as given in Table. 2.2 and Table. 2.3 respectively.

TABLE 2.2: State variables of the small-signal model of the converter

\begin{tabular}{c|c}
\hline \hline System & State Variables \\
\hline AC system & $\Delta I_{t R}, \Delta I_{t I}$ \\
\hline PLL & $\Delta \delta_{m}, \Delta x_{p l l}$ \\
\hline AC control system & $\Delta x_{p}, \Delta x_{q}, \Delta x_{i t d}, \Delta x_{i t q}$ \\
\hline Measurement filters & $\Delta P_{(m)}, \Delta Q_{(m)}$ \\
\hline DC control system & $\Delta x_{c a p}, \Delta x_{c l}, \Delta x_{i d c}$ \\
\hline DC system & $\Delta v_{d c}, \Delta i_{d c}$ \\
\hline
\end{tabular}

TABLE 2.3: Control inputs of the small-signal model of the converter

\begin{tabular}{c|c}
\hline \hline System & Control inputs \\
\hline AC system & $\Delta V_{s R}, \Delta V_{s I}$ \\
\hline Control system & $\Delta P_{(r e f)}, \Delta Q_{(r e f)}$ \\
\hline
\end{tabular}

Fig. 2.10 illustrates the block diagram of the developed overall state space model of the converter which indicates the flow of signals between each component of the system. 


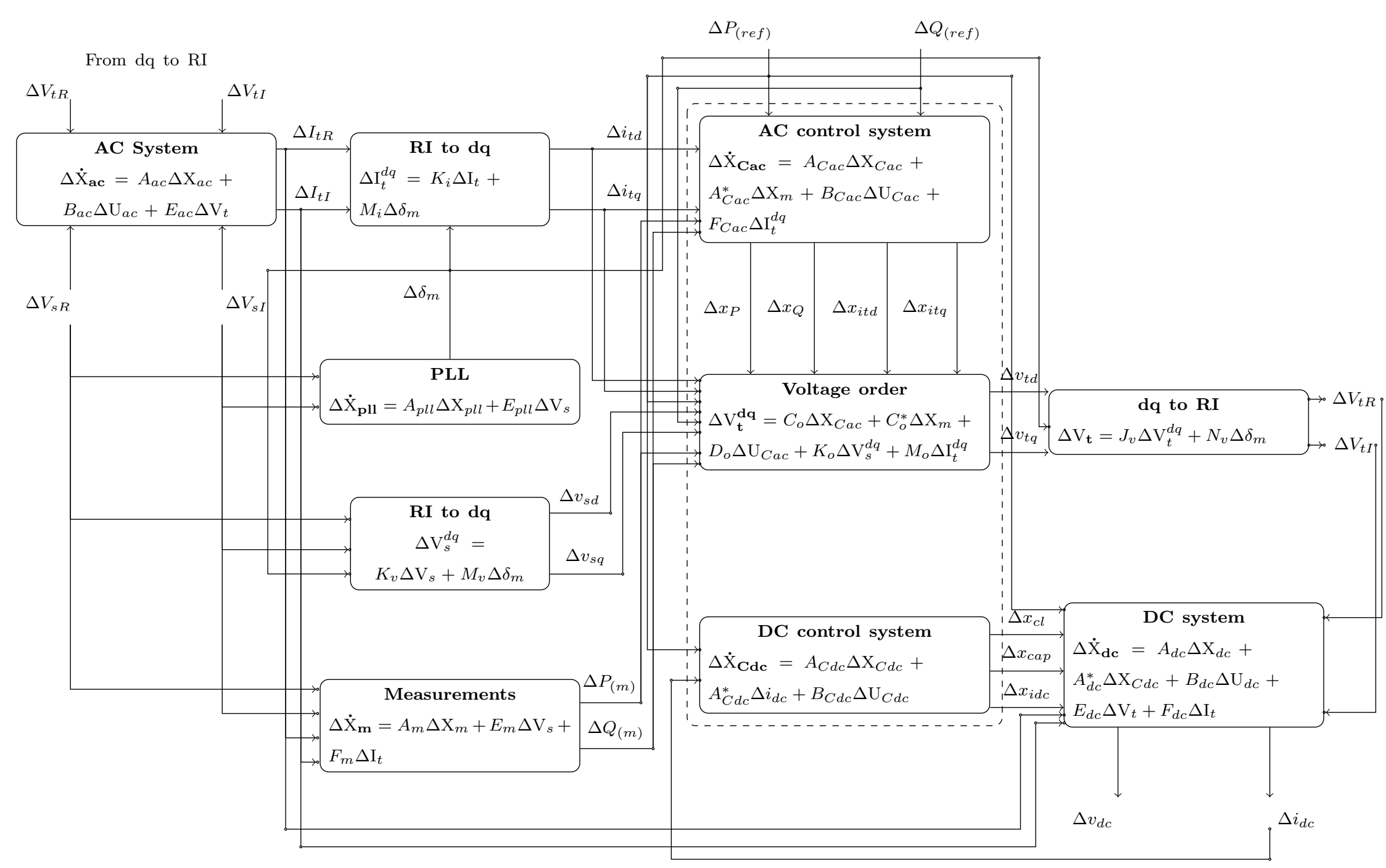

FIGURE 2.10: $15^{\text {th }}$ order small signal model of the hybrid modular multilevel VSC 


\subsection{Validation of the Overall State-Space Model of the Converter}

The small signal model of the converter was obtained by linearizing non-linear dynamic equations governing the behaviour of each converter component around an equilibrium point as explained in the previous section. It is thus necessary to validate this against a detailed non-linear model for accuracy, prior to be used for a stability analysis of this novel converter topology.

This has been achieved in the thesis with the developed EMT simulation model using PSCAD/EMTDC, explained in 2.1. The simulation parameters specified in Table 2.1 were used for this validation and the PI-controller gains are as given in Table 2.4. These proportional and integral gains have been obtained using the trail-and-error method for controller tuning.

TABLE 2.4: PI controller gains of the test system

\begin{tabular}{l|c|c|c}
\hline \hline & Controller & $K_{P}$ & $K_{I}$ \\
\hline \multirow{2}{*}{ Outer-loop (ac controls) } & PLL & 50 & 900 \\
\cline { 2 - 4 } & Active Power & 0.009 & 1.25 \\
\hline \multirow{2}{*}{ Inner-loop (ac controls) } & Reactive Power & 0.5 & 50 \\
\cline { 2 - 4 } & $d$-axis current & 4 & 500 \\
\hline Outer-loop (dc controls) & Submodule capacitor voltage & 5 & 50 \\
\hline Inner-loop (dc controls) & DC link current & 0.001 & 20 \\
\hline
\end{tabular}

The equilibrium operating point of the converter has been selected to be $P_{a c}=1.0$ $\mathrm{pu}$ and $Q_{a c}=0 \mathrm{pu}$ around which the linearization of the dynamic equations of the converter and the associated controls has been performed in order to obtain the small-signal model derived in 2.2. Validation of the small signal model was performed by applying a small disturbance to active and reactive power reference 
settings $\left(P_{(r e f)}, Q_{(r e f)}\right)$ given to the outer-loop control of the decoupled $d q$ control scheme, one at a time and comparing results obtained from the two models against one another. The perturbation applied has a magnitude of $0.1 \mathrm{pu}$ which is maintained for a period of $100 \mathrm{~ms}$.

\subsubsection{Disturbance applied to the active power reference}

The comparison of results obtained from the EMT model and the developed smallsignal model is illustrated in Fig. 2.11 for a disturbance given to the active power reference $\left(P_{(r e f)}\right)$ at $t=2.0 \mathrm{~s}$ for a period of $100 \mathrm{~ms}$, with a $10 \%$ change in magnitude. The results show the traces for the variation in active power $\left(\Delta P_{(a c)}\right)$ in a time window of $700 \mathrm{~ms}$.

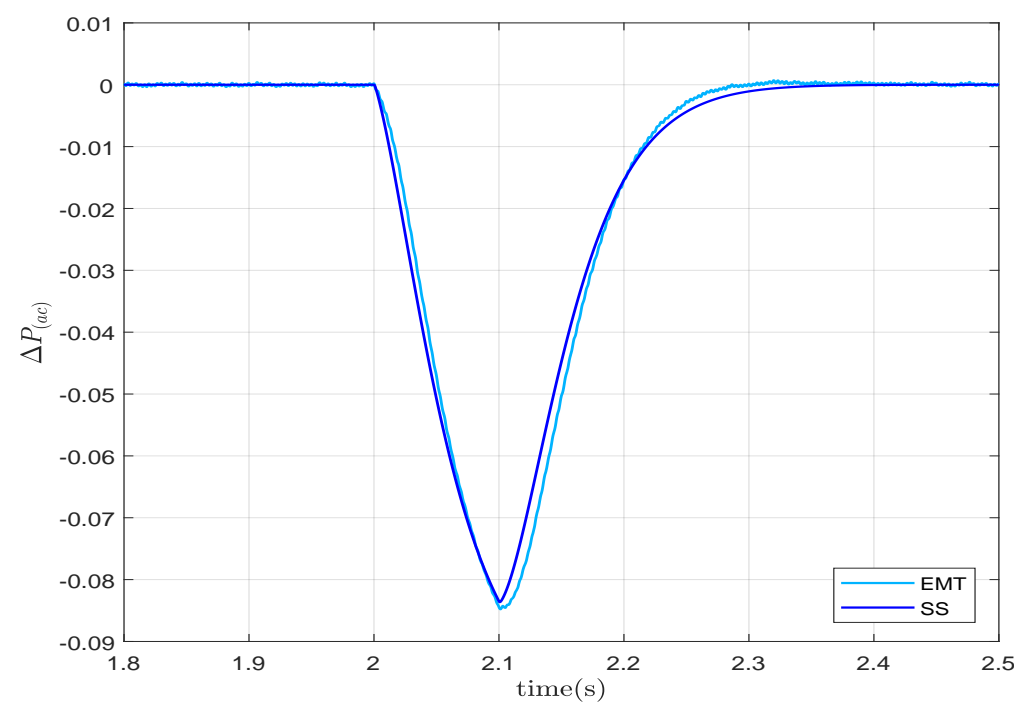

FIGURE 2.11: Variation in active power $\left(\Delta P_{(a c)}\right)$ for a small perturbation in respective reference $P_{(r e f)}$ 


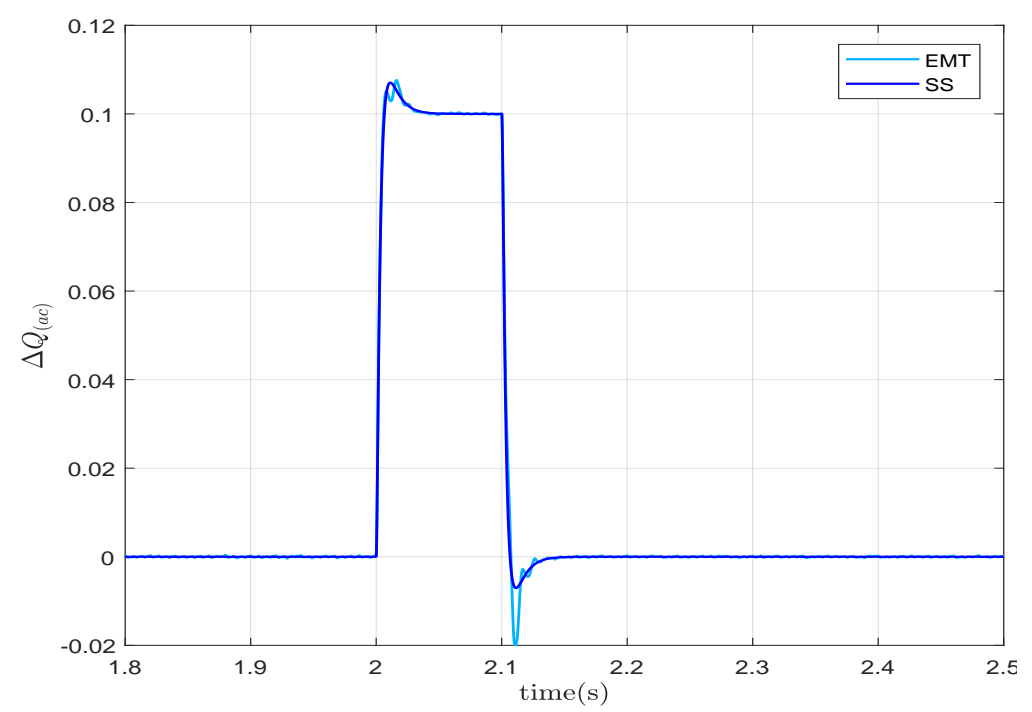

FiguRE 2.12: Variation in reactive power $\left(\Delta Q_{(a c)}\right)$ for a small perturbation in respective reference $Q_{(r e f)}$

\subsubsection{Disturbance applied to the reactive power reference}

The comparison of results obtained from the EMT model and the developed smallsignal model is illustrated in Fig. 2.12 for a disturbance given to the reactive power reference $\left(Q_{(r e f)}\right)$ at $t=2.0 \mathrm{~s}$ for a period of $100 \mathrm{~ms}$, with a $10 \%$ change in magnitude. The results show the traces for the variation in reactive power $\left(\Delta Q_{(a c)}\right)$ in a time window of $700 \mathrm{~ms}$.

As given in Fig. 2.11, Fig. 2.12 the trace obtained from the small-signal model accurately follows that of the detailed EMT model, hence validating the developed model. 


\subsection{Modified Overall State-Space Model of the Hybrid Modular Multilevel Converter}

The dc voltage applied across the composite chain-link arrangement of the converter is obtained through the summation of the rectified sinusoid references for the three phases; synthesized to fulfil the demand of the connected ac network. The variations in this dc voltage were neglected while developing the small-signal model in 2.2 and instead a steady state dc voltage of $V_{G}$ has been assumed. Thus the impact of the dc voltage on the accuracy of the derivation of the small-signal model has been further studied in this section.

The decoupled- $d q$ controller generates the desired converter voltage magnitude $\widehat{v_{t}}=\sqrt{v_{t d}^{2}+v_{t q}^{2}}$ according to the active and reactive power setting provided. Hence for a given $M I$, variations in the actual dc voltage of the converter can be accounted in the derivation of its small-signal model as given from Equation (2.44) and (2.45). $v_{t d}^{*}$ and $v_{t q}^{*}$ are the modified voltage orders of the converter with the variations in the dc-link voltage incorporated into the derivation of the small-signal model.

$$
\begin{aligned}
& v_{t d}^{*}=\frac{v_{t d} v_{d c}}{V_{G}} \\
& v_{t q}^{*}=\frac{v_{t q} v_{d c}}{V_{G}}
\end{aligned}
$$

The linearized algebraic equations that explain the relationship between the dclink voltage and the voltage orders generated by the converter could be obtained as given in Equation (2.46)

$$
\Delta \mathrm{V}_{t}^{d q *}=C_{m} \Delta \mathrm{V}_{t}^{d q}+D_{m} \Delta v_{d c}
$$


where

$$
\begin{aligned}
\Delta \mathrm{V}_{t}^{d q *} & =\left[\Delta v_{t d}^{*}, \Delta v_{t q}^{*}\right] \\
\Delta \mathrm{V}_{t}^{d q} & =\left[\Delta v_{t d}, \Delta v_{t q}\right] \\
C_{m} & =\left[\begin{array}{cc}
\frac{V_{d c 0}}{V_{G}} & 0 \\
0 & \frac{V_{d c 0}}{V_{G}}
\end{array}\right] \\
D_{m} & =\left[\begin{array}{l}
\frac{v_{t d 0}}{V_{G}} \\
\frac{v_{t q 0}}{V_{G}}
\end{array}\right]
\end{aligned}
$$

Applying $d q$-transformation to the modified voltage orders $\left(v_{t d}^{*}, v_{t q}^{*}\right)$ obtained from Equation (2.46), results in the respective voltage components in the $R I$ co-ordinate frame which are then integrated with the state-space models derived for the rest of the converter components following the procedure explained in 2.2.1-2.2.7. The block diagram of the overall modified state space model of the hybrid converter is illustrated in Fig. 2.13 explaining the signal flows between the converter components. 


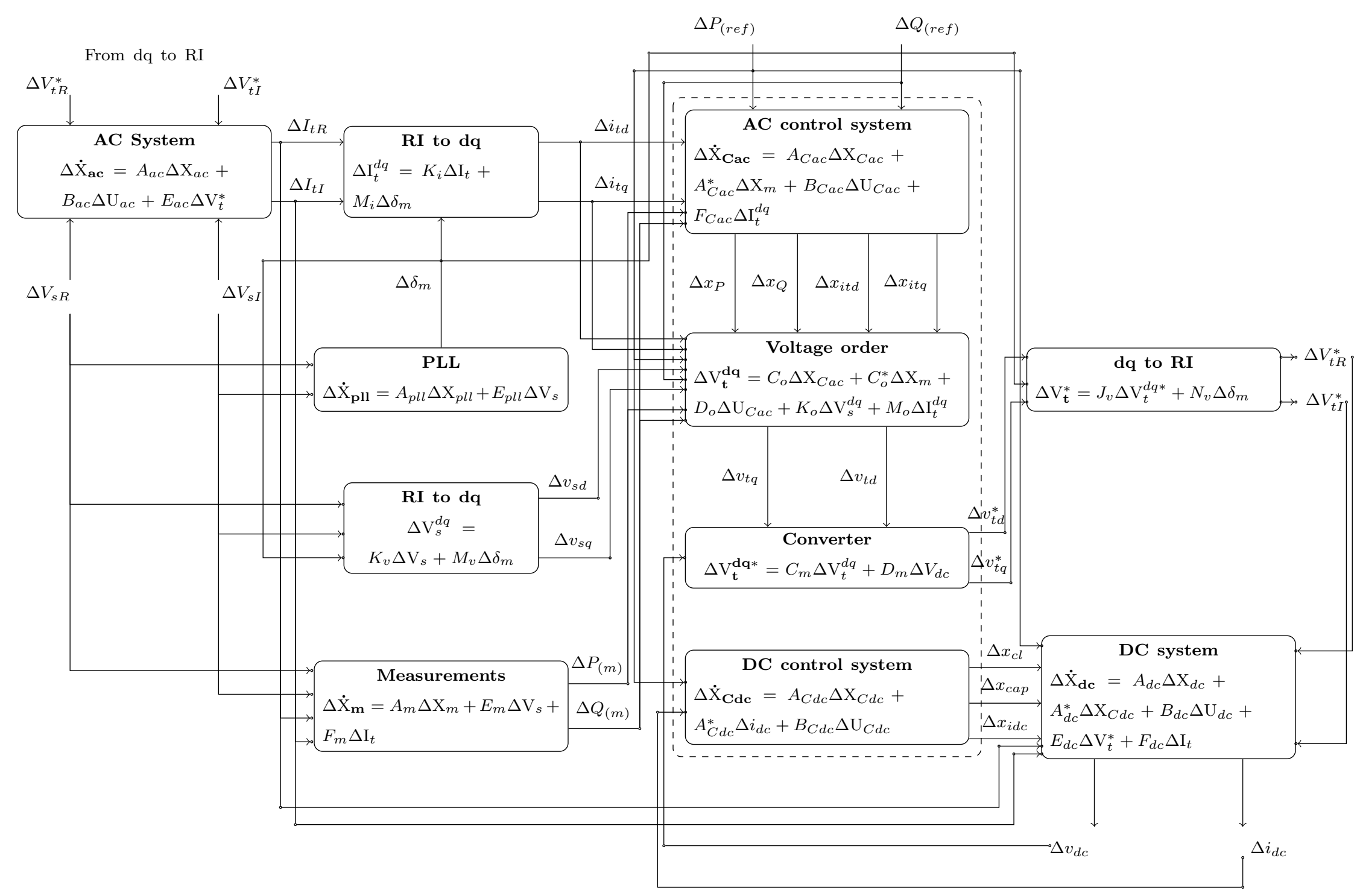

FiguRE 2.13: Modified small signal model of the hybrid modular multilevel VSC 


\subsubsection{Validation of the Modified Small-Signal Model of the Converter}

Similarly to the model developed in 2.2 , the modified small-signal model obtained in 2.4 should be validated against a detailed non-linear simulation for accuracy. The steps explained in 2.3 were followed in order to validate the modified smallsignal model for which a small disturbance with a magnitude of $0.1 \mathrm{pu}$ persisting for $100 \mathrm{~ms}$ is applied to the active and reactive power reference settings of the decoupled- $d q$ control scheme. Results obtained from the two models have been compared as explained below.

\subsubsection{Disturbance applied to the active power reference}

The comparison of results obtained from the EMT model and the developed smallsignal model is illustrated in Fig. 2.14 for a disturbance given to the active power reference $\left(P_{(r e f)}\right)$ at $t=2.0 \mathrm{~s}$ for a period of $100 \mathrm{~ms}$, with a $10 \%$ change in magnitude. The results show the traces for the variation in active power $\left(\Delta P_{(a c)}\right)$ in a time window of $700 \mathrm{~ms}$.

\subsubsection{Disturbance applied to the reactive power reference}

The comparison of results obtained from the EMT model and the developed smallsignal model is illustrated in Fig. 2.15 for a disturbance given to the reactive power reference $\left(Q_{(r e f)}\right)$ at $t=2.0 \mathrm{~s}$ for a period of $100 \mathrm{~ms}$, with a $10 \%$ change in magnitude. The results show the traces for the variation in reactive power $\left(\Delta Q_{(a c)}\right)$ in a time window of $700 \mathrm{~ms}$. 


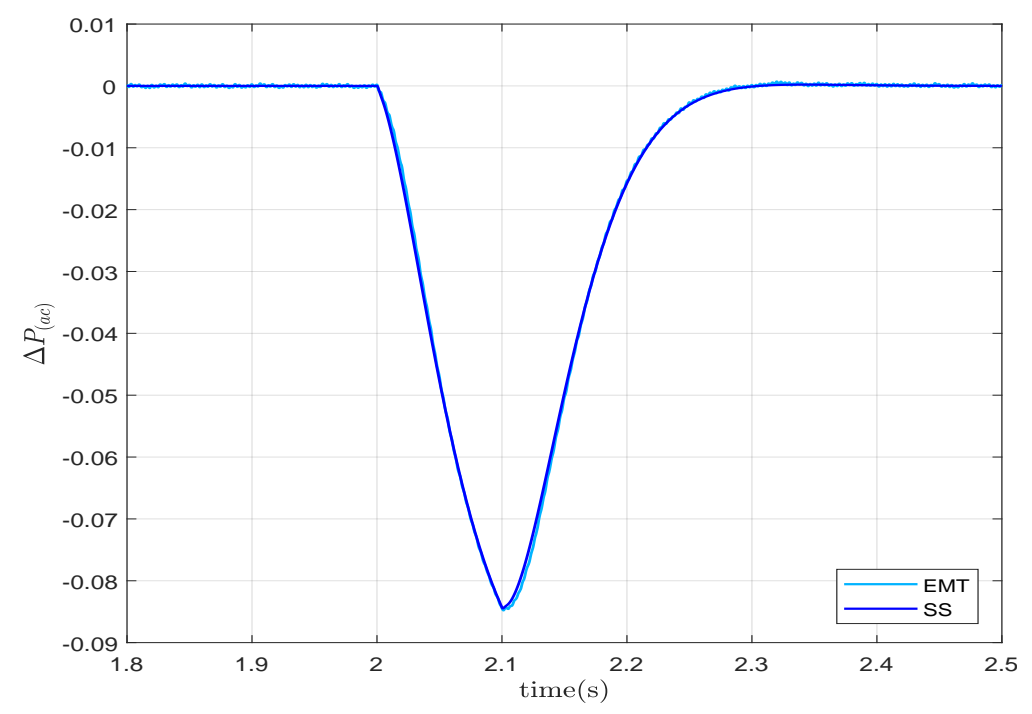

FiguRE 2.14: Variation in active power $\left(\Delta P_{(a c)}\right)$ for a small perturbation in respective reference $P_{(r e f)}$

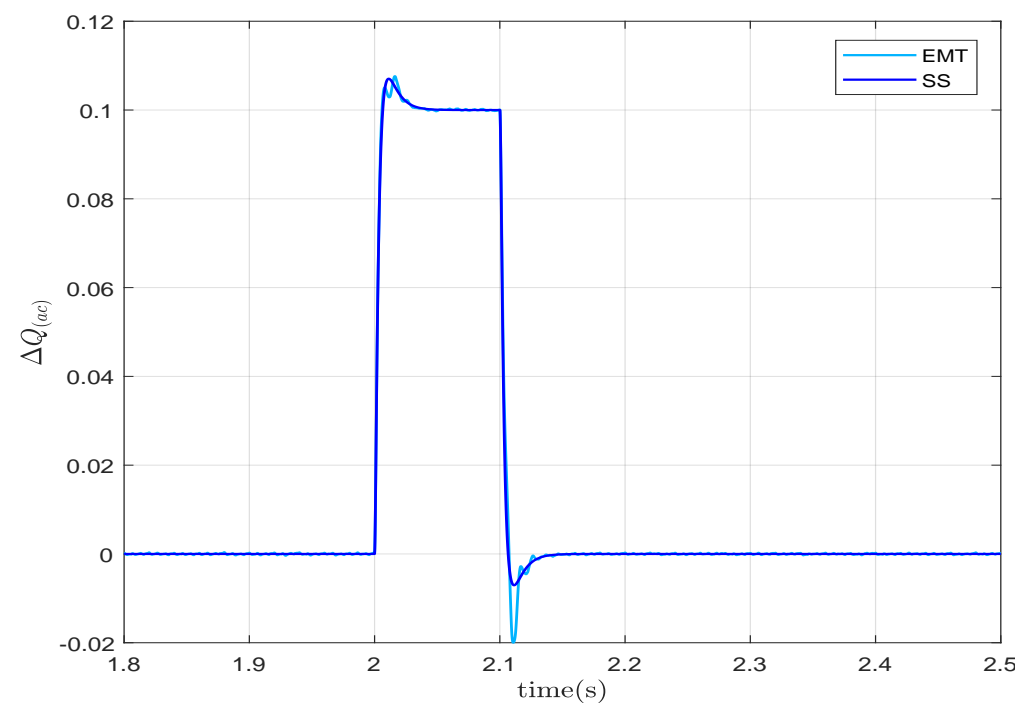

FiguRE 2.15: Variation in reactive power $\left(\Delta Q_{(a c)}\right)$ for a small perturbation in respective reference $Q_{(r e f)}$

The results obtained from the detailed non-linear model and the modified linearized small-signal model presented above show close correspondence as expected. However the accuracy of fit between the traces of the EMT model and modified small-signal model, for the variation in active power is better compared to the previous case, in which the variations in the dc-link voltage has not been considered. Besides, variation in reactive power does not show a significant improvement 
in the accuracy of fit between the traces obtained from the EMT model and the modified small-signal model compared to the former case.

The control circuitry regulates the dc-link voltage in the close proximity of its desired value $V_{G}$ through triplen harmonic voltage compensation. Thus the impact of the variations in the dc-link voltage on the accuracy of the derived small-signal model is not significant which is further observed from the comparison of results obtained from the state-space models with the non-linear EMT simulation model. Further, results of a detailed eigenvalue analysis performed on the state matrix of the modified small-signal model following the procedure presented in the next chapter of the thesis confirmed that the dc-link voltage has a minimal impact on the accuracy of the derivation of the small-signal model for the novel hybrid converter configuration.

\section{Chapter Summary}

This chapter described the development of a small-signal model for the stability evaluation of the novel converter topology. The non-linear dynamic equations have been linearized using the Taylor series expansion, assuming the perturbations from the equilibrium state are small enough for a linear model to be valid. The overall state-space model developed has been validated against a detailed non-linear transient simulation model and the results from the two exhibit close correspondence. Variations in the mean composite chain-link voltage are incorporated by modifications applied to the state-space formulation of the converter. 


\section{Chapter 3}

\section{Stability Analysis of the Hybrid Modular Multilevel VSC}

The state-space form of the converter given in Equation (3.1) has been obtained by linearizing non-linear dynamic equations governing the behaviour of the system about an equilibrium operating point. Thus the linearity of the model will be valid for small perturbations around this point.

$$
\Delta \dot{\mathrm{X}}=A \Delta \mathrm{X}+B \Delta \mathrm{U}
$$

where $\Delta \mathrm{X}$ is the state vector of dimension $n$

$\Delta \mathrm{U}$ is the input vector of dimension $r$

A is the state matrix of size $n \times n$

$\mathrm{B}$ is the input matrix of size $n \times r$

Well defined techniques available for analysing linear systems could be used for the stability assessment of the converter which include root-locus, Bode plot, Nyquist 
criterion and eigenvalue (state-space) analysis. A stability assessment on the developed model using a technique listed above provides an insight to the performance of the system in terms of to its stability and controllability aspects. This further facilitates the design of controllers to improve the stability thereby securing reliable power transmission using the selected converter topology.

In order to assess the small-signal stability of the hybrid modular multilevel converter this thesis uses the eigenvalue analysis technique which has a variety of applications in power system control studies. This provides stability related frequency domain attributes such as eigenvalues (modes), frequency and damping of the oscillatory modes present, eigenvectors and participation of state variables in each mode thereby giving a better insight to the behaviour of the system.

The linearized state-space model for the converter, developed and validated in 2.2 is used for this stability analysis. The following section provides a brief introduction to the eigenproperties of the state-space system.

\subsection{Eigenproperties and Stability Criterion}

\subsubsection{Eigenvalues}

Eigenvalues of the state matrix A are given by the values of $\lambda$ from the Equation (3.2).

$$
\operatorname{det}(A-\lambda I)=0
$$

where $\lambda=\left[\lambda_{1}, \lambda_{2}, \ldots \lambda_{n}\right]$ are eigenvalues of the matrix A. These eigenvalues could be either real or complex. If $\mathrm{A}$ is real these complex eigenvalues occur in conjugate pairs. 
The time evolution of a mode corresponding to an eigenvalue $\lambda_{i}$ is given by $e^{\lambda_{i} t}$. Thus,

(a) "A real eigenvalue corresponds to a non-oscillatory mode. A negative real eigenvalue represents a decaying mode. The larger its magnitude, the faster the decay. A positive real eigenvalue represents aperiodic instability."

(b) "Complex eigenvalues occur in conjugate pairs, and each pair corresponds to an oscillatory mode." [16]

The small-signal stability of the system could be analysed from the eigenvalues of the state matrix and the criterion for stability of a system described below is based on the Lyapunov's first method [16].

(i) "When the eigenvalues have negative real parts, the original system is asymptotically stable."

(ii) "When at least one of the eigenvalues has a positive real part, the original system is unstable."

(iii) "When the eigenvalues have real parts equal to zero, it is not possible on the basis of the first approximation to say anything in the general."

A complex conjugate pair of eigenvalues could be expressed as in Equation (3.3).

$$
\lambda=\sigma \pm j \omega
$$

The real part $\sigma$ gives damping ratio $(\zeta)$ while the imaginary component $\omega$ gives the frequency of oscillation $(f)$ which could be obtained as shown below from Equation (3.4) and (3.5) respectively. The damping ratio determines the rate of decay of the amplitude of the oscillation and a negative real part represents a damped oscillation where as a positive real part corresponds to an oscillation with 
growing amplitude.

$$
\begin{gathered}
\zeta=\frac{-\sigma}{\sqrt{\sigma^{2}+\omega^{2}}} \\
f=\frac{\omega}{2 \pi}
\end{gathered}
$$

\subsubsection{Eigenvectors}

For a given eigenvalue $\lambda_{i}$, the $n$-column vector $\phi_{i}$ that satisfies Equation (3.6) given below is defined as the right eigenvector of $\lambda_{i}$.

$$
A \phi_{i}=\lambda_{i} \phi_{i} \quad i=1,2, \ldots n
$$

where

$$
\phi_{i}=\left[\begin{array}{c}
\phi_{1 i} \\
\phi_{2 i} \\
\cdot \\
\cdot \\
\phi_{n i}
\end{array}\right]
$$

For a given eigenvalue $\lambda_{i}$, the $n$-row vector $\psi_{i}$ that satisfies Equation (3.7) given below is defined as the left eigenvector of $\lambda_{i}$.

$$
\begin{gathered}
\psi_{i} A=\lambda_{i} \psi_{i} \quad i=1,2, \ldots n \\
\psi_{i}=\left[\psi_{i 1}, \psi_{i 2}, . ., \psi_{i n}\right]
\end{gathered}
$$




\subsubsection{Participation Factor}

The participation factor calculated for each mode (eigenvalue) combining the corresponding right and left eigenvectors provides a measurement of the association between the state variables and the mode of interest [16]. Participation matrix for the $i^{\text {th }}$ eigenvalue $\lambda_{i}$ is obtained from Equation (3.8)

$$
p_{i}=\left[\begin{array}{c}
p_{1 i} \\
p_{2 i} \\
\cdot \\
p_{n i}
\end{array}\right]=\left[\begin{array}{c}
\phi_{1 i} \psi_{i 1} \\
\phi_{2 i} \psi_{i 2} \\
\cdot \\
\phi_{n i} \psi_{i n}
\end{array}\right]
$$

$p_{k i}=\phi_{k i} \psi_{i k}$ is referred to as the participation factor which represents the participation of the $k^{\text {th }}$ state variable in $i^{\text {th }}$ mode, where $\phi_{k i}$ denotes the $k^{\text {th }}$ entry of the right eigenvector $\phi_{i}$ while $\psi_{i k}$ refers to the $k^{\text {th }}$ entry of the left eigenvector $\psi_{i}$.

\subsection{Eigenvalue Analysis of the Test System}

An eigenvalue analysis was performed on the state-space model developed and validated in the previous chapter with the intention of assessing the converter's "stability in small". Oscillatory modes corresponding to the eigenvalues obtained from the state matrix have been considered in this study. Further, a participation factor analysis has been conducted on these oscillatory modes observed in order to understand the contribution of each state of the system to the modes of interest. The study was conducted for two different system strengths of the converter connected ac network, thus to evaluate its impact on the stability and performance of the converter. 


\subsubsection{Analysing the impact of the system strength on the stability of the converter}

The strength of an ac system is parameterized by the index referred to as the Short Circuit Ratio (SCR) which can be calculated from the Equation (3.9) given below.

$$
S C R=\frac{\text { Short circuit } M V A}{\text { Rated DC power }}
$$

The Short Circuit MVA of the ac system is given by $\frac{V_{L L}^{2}}{\left|Z_{s}\right|}$ where $V_{L L}$ is the line-line voltage and $Z_{s}$ is the Thevenin equivalent impedance of the system which in this scenario is the impedance of series connected RL branch. An ac system having a high impedance is categorized as a weak system, hence has a low SCR whereas a low impedance system is considered to be strong, having a high SCR. Appropriate values were selected for $\mathrm{R}$ and $\mathrm{L}$ in order to model a moderately strong system with the $\mathrm{SCR}=4.0$ and a weak system with $\mathrm{SCR}=2.0$.

Eigenvalues of the state-matrix for the converter model having test parameters given in Table 2.1 and PI controller gains given in Table 2.4 were obtained. The oscillatory modes observed along with their respective frequency $(f)$ and damping ratio $(\zeta)$ are tabulated in Table 3.1 .

The ratio of the converter side winding voltage to that of the ac side for the transformer has been set to the value of 1:1.1 in the study presented in [4]. This ratio had to be changed to 1.1:1 between the converter side and the ac network side in order to eliminate the low frequency oscillations observed in the traces for active and reactive power quantities obtained from the EMT simulation, with the converter connected ac system having a low SCR. This oscillation was not reflected in the small-signal study hence was made a requirement to analyse these un-modelled dynamics of the converter. Observations made in these studies have 


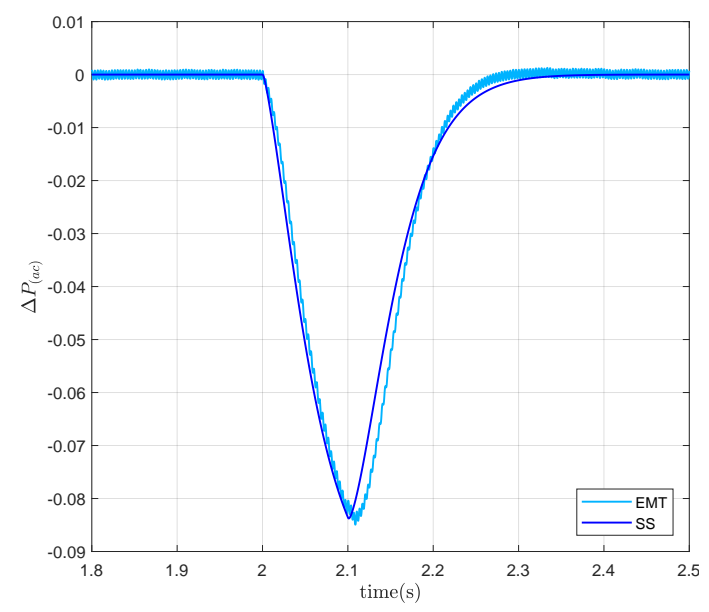

(I)

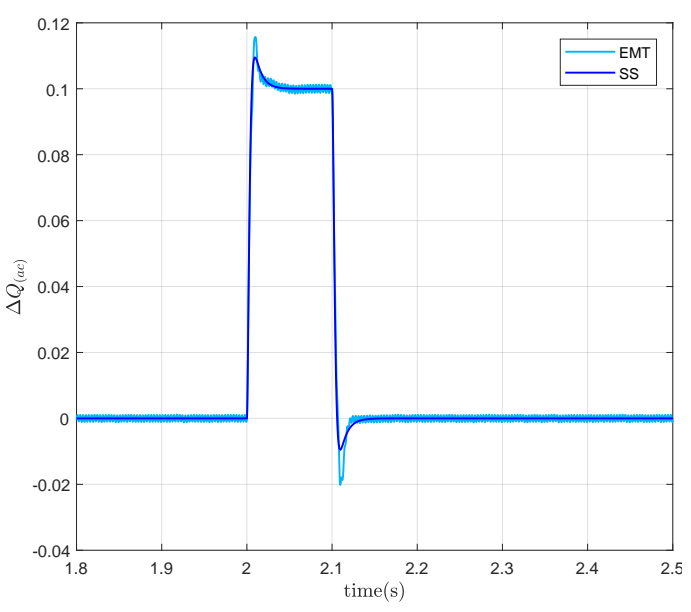

(II)

FiguRE 3.1: Comparison of results for the converter connected to a moderately strong ac system $(\mathrm{SCR}=4.0)$ (I) Variation in active power for a small disturbance in $P_{(r e f)}$ (II) Variation in reactive power for a small disturbance in $Q_{(r e f)}$

been further discussed with details in 3.4.

TABLE 3.1: Oscillatory modes for ac system strengths of $\mathrm{SCR}=4.0$ and $\mathrm{SCR}=2.0$

\begin{tabular}{c|c|c|c|c}
\hline \hline \multirow{2}{*}{ Mode } & \multicolumn{2}{|c|}{ SCR=4.0 } & \multicolumn{2}{c}{ SCR=2.0 } \\
\cline { 2 - 5 } & $\mathrm{f}(\mathrm{Hz})$ & $\zeta(\%)$ & $\mathrm{f}(\mathrm{Hz})$ & $\zeta(\%)$ \\
\hline Mode 1 & 58.17 & 68 & 58.17 & 68 \\
\hline Mode 2 & 30 & 93.9 & 49.98 & 58.45 \\
\hline Mode 3 & 2.64 & 83.8 & 2.64 & 83.8 \\
\hline Mode 4 & 1.14 & 96.9 & 1.08 & 97.27 \\
\hline Mode 5 & - & - & 14.2 & 92 \\
\hline
\end{tabular}

A comparison between the results obtained from the developed small-signal model and the EMT simulation model for a small disturbance in $P_{(r e f)}, Q_{(r e f)}$ for the two ac system strengths $(\mathrm{SCR}=4.0$ and $\mathrm{SCR}=2.0)$ are given in Fig. 3.1 and Fig. 3.2 respectively.

It can be observed that all the oscillatory modes have high damping. Damping of Mode 2 decreases significantly with the reduction of system strength, yet remains at a satisfactory level. Further, it can be noticed that low frequency modes are 


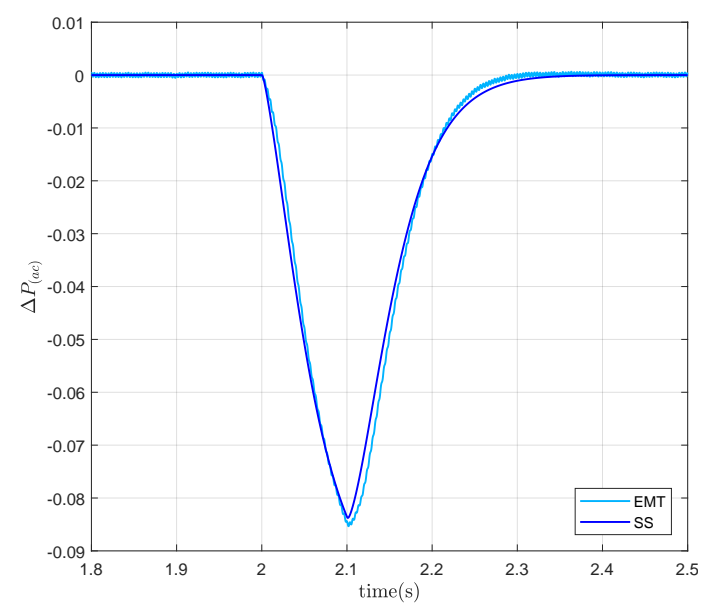

(I)

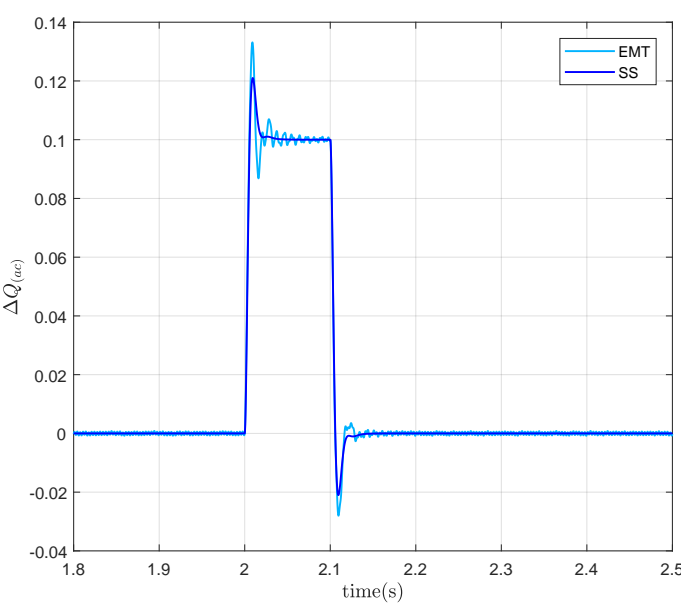

(II)

FiguRE 3.2: Comparison of results for the converter connected to a moderately strong ac system $(\mathrm{SCR}=2.0)$ (I) Variation in active power for a small disturbance in $P_{(r e f)}$ (II) Variation in reactive power for a small disturbance in $Q_{(r e f)}$

well damped in both scenarios. Hence stability of low frequency oscillation modes in not a concern. An additional oscillatory mode is observed when the converter is connected to a weak ac system having a SCR of 2.0.

\subsubsection{Participation Factor Analysis}

The participation factor analysis gives a further insight to the eigenvalues of the state-matrix of the developed linearized state-space model. Participation factor $p_{k i}$ of a mode corresponding to eigenvalue $\lambda_{i}$ is a measure of the influence of the $k^{\text {th }}$ state on the $i^{\text {th }}$ mode as explained in 3.1.3.

Such analysis has been conducted on the eigenvalues of the state matrix of the developed small-signal model for the selected system strengths of the ac network $(\mathrm{SCR}=4.0$ and $\mathrm{SCR}=2.0)$. The participation factors corresponding to the oscillatory modes are as illustrated below. 


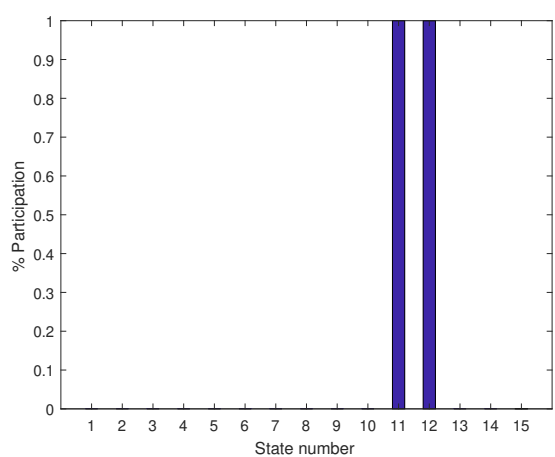

(I) Mode 1

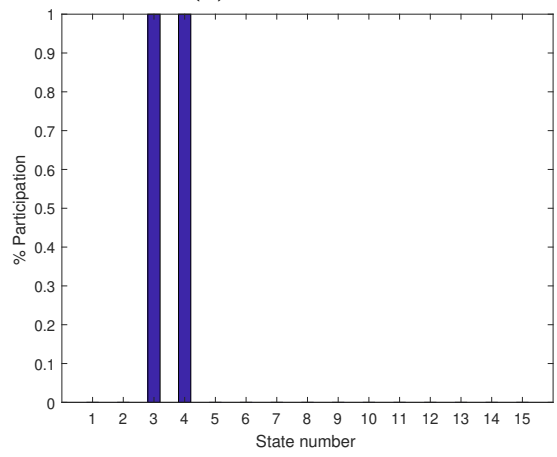

(III) Mode 3

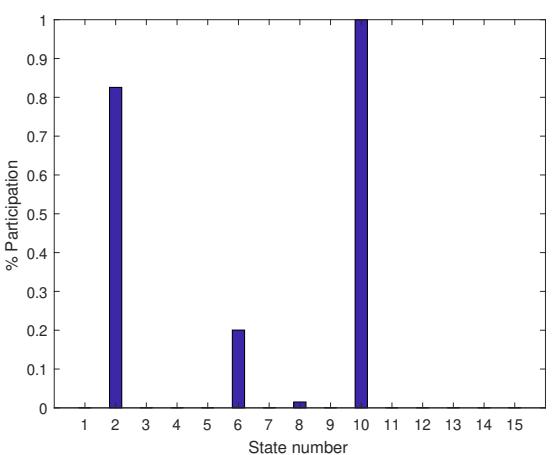

(II) Mode 2

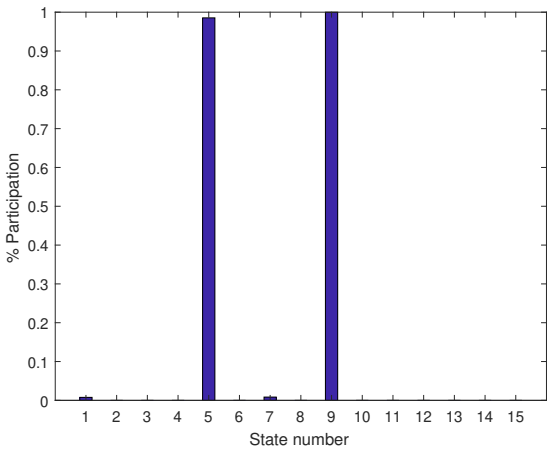

(IV) Mode 4

FIGURE 3.3: Participation factors of oscillatory modes for $\mathrm{SCR}=4.0$

Participation factors of the oscillatory modes with the converter connected to a moderately strong system $(\mathrm{SCR}=4.0)$ are given in Fig. 3.3 with the order of state variables given in Table. 3.2.

TABLE 3.2: Order of states of the small-signal model

\begin{tabular}{c|c|l}
\hline \hline State Number & System & States \\
\hline 1,2 & AC system & $\Delta I_{t R}, \Delta I_{t I}$ \\
\hline 3,4 & PLL & $\Delta x_{p l l}, \Delta \delta_{m}$ \\
\hline $5-8$ & AC control system & $\Delta x_{p}, \Delta x_{q}, \Delta x_{i d}, \Delta x_{i q}$ \\
\hline 9,10 & Measurement filters & $\Delta P_{(m)}, \Delta Q_{(m)}$ \\
\hline 11,12 & DC system & $\Delta v_{d c}, \Delta i_{d c}$ \\
\hline $13-15$ & DC control system & $\Delta x_{c a p}, \Delta x_{c l}, \Delta x_{i d c}$ \\
\hline
\end{tabular}

From the results obtained in the participation factor analysis on a converter connected to a strong ac system, it could be deduced that Mode 1 is excited by the dc system states whereas Mode 3 exhibits the dynamics governed by the PLL of the 
converter. State variables related to the reactive power of the ac system and the vector control scheme participate significantly in Mode 2 whereas the states governing that of the active power controller are observed to be highly participating in Mode 4.

Table 3.3 presents the categorization of the oscillatory modes based upon the participation factor analysis.

TABLE 3.3: Classification of modes for $\mathrm{SCR}=4.0$

\begin{tabular}{c|c|c}
\hline \hline Mode & \multicolumn{2}{|c}{ Classification } \\
\hline 1 & dc resonance & \multirow{2}{*}{ Network mode } \\
\hline 2 & Reactive power controller & \multirow{2}{*}{ Controller modes } \\
\hline 3 & PLL & \\
\hline 4 & Active power controller &
\end{tabular}

A similar participation factor analysis was conducted on the small-signal model obtained with the converter connected to a weak ac system having a SCR of 2.0 and the results are given in Fig. 3.4.

Observations of the participation factor analysis with the converter connected to a weak ac network are similar to that of the previous scenario with a higher system strength. The additional mode present in this case is dominantly participated by the states governing the current controller of the converter. Table 3.4 presents the categorization of the oscillatory modes with the converter connected to a weak ac network.

TABle 3.4: Classification of modes for $\mathrm{SCR}=2.0$

\begin{tabular}{c|c|c}
\hline \hline Mode & Classification \\
\hline 1 & dc resonance & \multirow{2}{*}{ Network mode } \\
\hline 2 & Reactive power controller & \multirow{2}{*}{ Controller modes } \\
\hline 3 & PLL & \\
\hline 4 & Active power controller & \\
\hline 5 & Current controller & \\
\hline
\end{tabular}




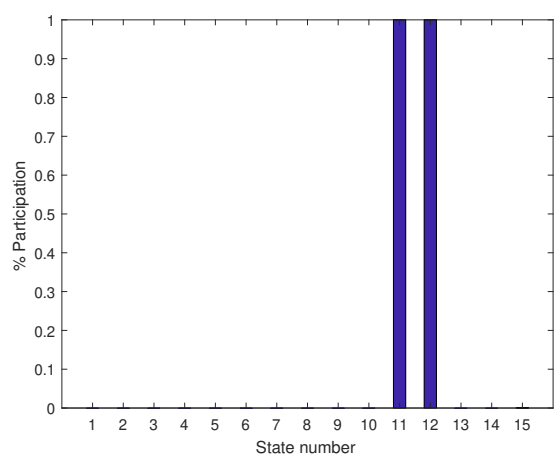

(I) Mode 1

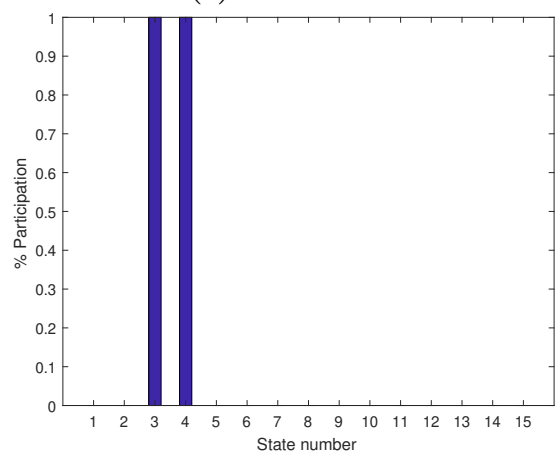

(III) Mode 3

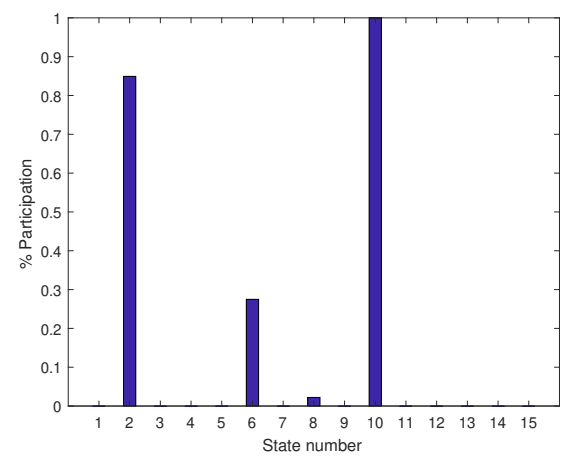

(II) Mode 2

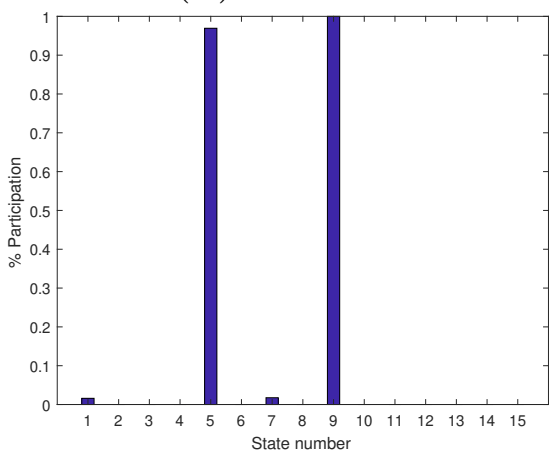

(IV) Mode 4

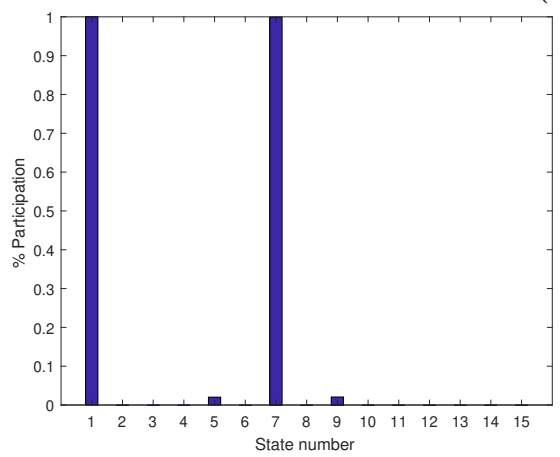

(v) Mode 5

FiguRE 3.4: Participation factors of oscillatory modes for $\mathrm{SCR}=2.0$ 


\subsection{Sensitivity of the Small-Signal Stability of the Converter to Controller Gains}

\subsubsection{Impact of the PLL gains}

Studies conducted on VSCs that are connected to a weak ac system have revealed that the gains of the SRF-PLL which is used to provide angle information of the ac voltage to the controls may have an impact on the stability and performance of the converter $[20],[21],[22]$. These studies have further proposed the use of alternative synchronization techniques which eliminate the use of a PLL thereby removing the effect of its dynamics on the stability of the converter.

In order to evaluate the effects of the PLL gains on the stability of the converter, an analysis was performed by varying the proportional gain $K_{P(p l l)}$ from 10 to 200 in steps of 10 while the integral gain $K_{I(p l l)}$ was set to be $5 K_{P(p l l)}$ (thus varied from 50 to 1000). The stability of the system was evaluated observing the trajectories of the two modes having high contribution from the states of the PLL, in the complex plane. The remaining oscillatory modes were observed to be unaffected by the variation of the PLL gains.

The procedure followed with a converter connected to a strong ac system ( $\mathrm{SCR}=4.0$ ) resulted in the root loci of the two modes that are dominantly participated by the state variables of the PLL, given in Fig. 3.5.

The procedure was repeated for a system with the connected ac network having a SCR of 2.0 and the results obtained from the root loci of the modes in which the state variables of the PLL have a high participation are given in Fig. 3.6.

From the resultant loci, it could be observed that one of the modes is moving 


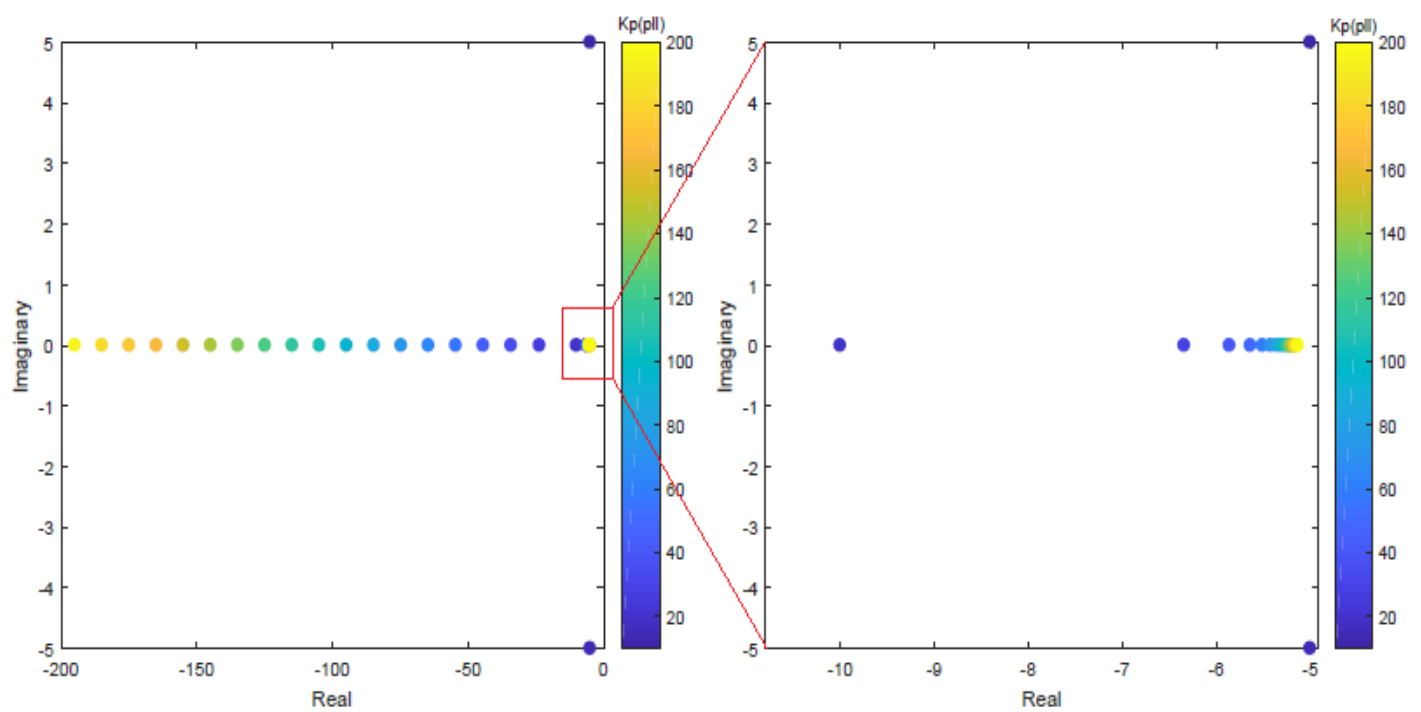

Figure 3.5: Trajectories of critical modes (second mode enlarged) for a change in PLL gains with moderately high SCR (=4.0) of the connected ac network

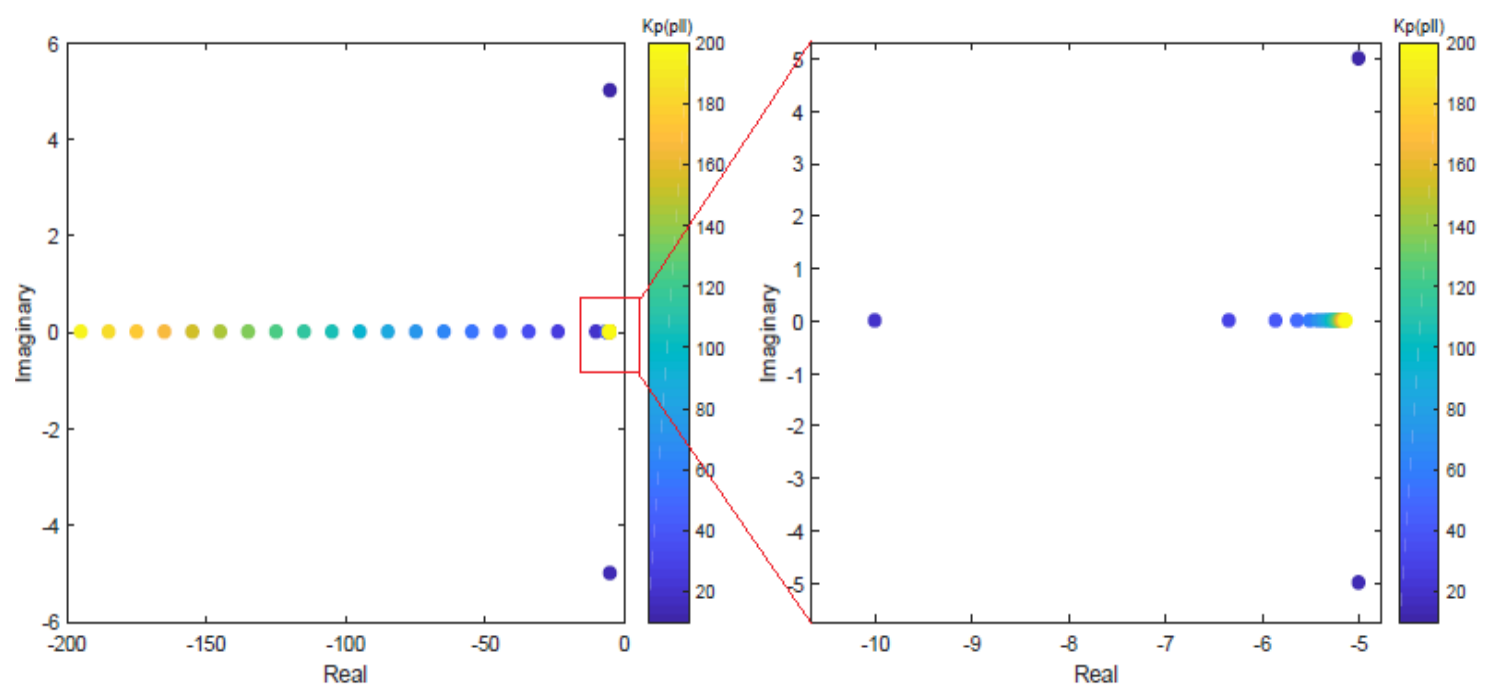

Figure 3.6: Trajectories of critical modes (second mode enlarged) for a change in PLL gains with weak SCR $(=2.0)$ of the connected ac network

towards the more stable region of the complex plane while the other mode is moving in the opposite direction. However, the converter remains stable for all values of $K_{P(p l l)}$ when connected to both strong and weak ac systems. The converter is driven to instability for infinitely large values of $K_{P(p l l)}$ which are not considered 
to be realistic values for gains of the PI controllers. Thus it could be concluded that the converter is stable for all practical values of $K_{P(p l l)}$, under both strong and weak ac network conditions.

\subsubsection{Sensitivity to measurement delays of $d q$ voltages and currents}

The VSC topologies are identified to have the capability of connecting with very weak ac systems which has not been possible with the conventional line-commutated converters. Besides this expectation, power engineers face challenges in connecting VSCs with weak ac networks [23]. Relevant studies that are presented in [20],[24], $[25],[26],[27],[28]$ identify the interactions of the decoupled $d q$ control scheme as the cause.

The $d q$ components of current and voltage quantities for the inner-loop control and voltage feed-forward of the ac control scheme are obtained through transducers, which introduce delays into these measurements. It is necessary to evaluate the impact of these measurement delays on the stability of the converter. This could be analysed through the sensitivity of the eigenvalues obtained from the state-matrix of a developed linearized small-signal model for the converter to changes in these measurements. Previous research discussed in [24],[26],[27],[28] have studied the influence of the inner-loop controller parameters on the stability of the converter, but the sensitivity of the measurement delays associated with decomposed voltage and current quantities has not been taken into account. Ref [25] has incorporated the effect of these measurement delays on the resonance stability of the VSC in the developed impedance model. 


$$
Y_{\alpha} \rightarrow \frac{1}{1+s T_{\alpha}} \rightarrow Y_{\alpha(m)}
$$

Figure 3.7: Measurement filter block

In this thesis, a study was conducted to evaluate the impact of the measurement delays associated with the $d q$-axis quantities of the inner-loop control on the stability of the converter.

The linearized model developed in 2.2 of Chapter 2 has incorporated only the measurement delays of the outer-loop quantities (i.e. active power and reactive power), but not of those for the inner-loop $d, q$ axis currents and voltages. Thus the small-signal model was modified with measurement blocks for $i_{t d}, i_{t q}, v_{s d}, v_{s q}$.

A first order filter was used to model the delay introduced by the transducer, similar to the measurement of active and reactive power quantities for the outerloop control as discussed in 2.2.3. The schematic of the filter block is given in Fig. 3.7 with the governing dynamic equation given in Equation (3.10).

$$
\dot{Y}_{\alpha(m)}=\frac{1}{T_{\alpha}}\left(Y_{\alpha}-Y_{\alpha(m)}\right)
$$

The schematic of the modified inner-loop control incorporating measurement delays for $d$ and $q$ axis currents and voltages is illustrated in Fig. 3.8. The equations governing the dynamics of the inner-loop control could be modified as given in Equation (3.11)-(3.16).

$$
\begin{aligned}
& \dot{i}_{t d(m)}=\frac{1}{T_{i d}}\left(i_{t d}-i_{t d(m)}\right) \\
& \dot{i}_{t q(m)}=\frac{1}{T_{i q}}\left(i_{t q}-i_{t q(m)}\right) \\
& \dot{v}_{s d(m)}=\frac{1}{T_{v d}}\left(v_{s d}-v_{s d(m)}\right)
\end{aligned}
$$




$$
\begin{gathered}
\dot{v}_{s q(m)}=\frac{1}{T_{v q}}\left(v_{s q}-v_{s q(m)}\right) \\
\dot{x}_{i t d}=\left(i_{t d(r e f)}-i_{t d(m)}\right) \\
\dot{x}_{i t q}=\left(i_{t q(r e f)}-i_{t q(m)}\right)
\end{gathered}
$$

Thus, by linearizing Equations (3.11)-(3.14) the small signal model of the current and voltage measurement delays could be obtained as given in Equation (3.17)(3.18).

$$
\begin{gathered}
\Delta \dot{\mathrm{X}}_{d i}=A_{d i} \Delta \mathrm{X}_{d i}+F_{d i} \Delta I_{t}^{d q} \\
\Delta \dot{\mathrm{X}}_{d v}=A_{d v} \Delta \mathrm{X}_{d v}+E_{d v} \Delta V_{s}^{d q}
\end{gathered}
$$

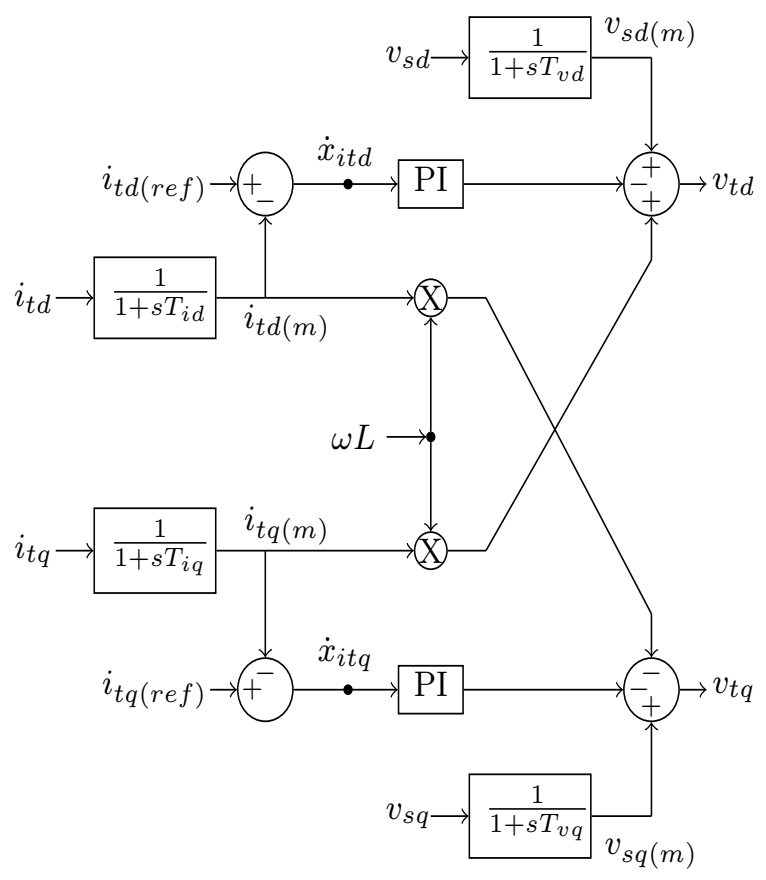

FiguRE 3.8: Inner-loop control with measurement delays included 
where

$$
\begin{aligned}
\Delta \mathrm{X}_{d i}= & {\left[\Delta i_{t d(m)}, \Delta i_{t q(m)}\right]^{T} } \\
\Delta \mathrm{X}_{d v}= & {\left[\Delta v_{s d(m)}, \Delta v_{s q(m)}\right]^{T} } \\
\Delta I_{t}^{d q}= & {\left[\Delta i_{t d}, \Delta i_{t q}\right]^{T} } \\
\Delta V_{s}^{d q}= & {\left[\Delta v_{s d}, \Delta v_{s q}\right]^{T} } \\
A_{d i}= & {\left[\begin{array}{cc}
-\frac{1}{T_{d i}} & 0 \\
0 & -\frac{1}{T_{q i}}
\end{array}\right] } \\
F_{d i}= & {\left[\begin{array}{cc}
\frac{1}{T_{d i}} & 0 \\
0 & \frac{1}{T_{q i}}
\end{array}\right] } \\
A_{d v}= & {\left[\begin{array}{cc}
-\frac{1}{T_{d v}} & 0 \\
0 & -\frac{1}{T_{q v}}
\end{array}\right] } \\
E_{d v}= & {\left[\begin{array}{cc}
\frac{1}{T_{d v}} & 0 \\
0 & \frac{1}{T_{q v}}
\end{array}\right] }
\end{aligned}
$$

Small time constants compared to those of active and reactive power which have a slow variation are used for the measurement of quantities such as instantaneous voltage and currents that vary faster. Furthermore the measurement filters for the $d$ and $q$ axis currents have the smallest time constants among all. Values of the time constants used for the measurement filters of outer and inner-loop variables are given in Table 3.5 .

TABLE 3.5: Time constants of the measurement filters of outer and innerloop controls

\begin{tabular}{c|c|c}
\hline \hline \multirow{2}{*}{ Outer-loop } & Controller & $\begin{array}{c}\text { Time } \\
\text { Constant (s) }\end{array}$ \\
\cline { 2 - 3 } & Active power & 0.02 \\
\hline \multirow{2}{*}{ Inner-loop } & Reactive power & 0.02 \\
\cline { 2 - 3 } & $d q$-axis voltages & 0.005 \\
\hline
\end{tabular}


The signal-flow diagram of the overall state-space model for the converter, modified to include the $d q$-axis current and voltage measurement delays is illustrated in Fig. 3.9. The modified system has 19 state variables and the order of states is given in Table 3.6.

TABLE 3.6: Order of states for the modified converter small-signal model to include measurement delays

\begin{tabular}{c|c|l}
\hline \hline $\begin{array}{c}\text { State } \\
\text { Number }\end{array}$ & System & States \\
\hline 1,2 & AC system & $\Delta I_{t R}, \Delta I_{t I}$ \\
\hline 3,4 & PLL & $\Delta x_{p l l}, \Delta \delta_{m}$ \\
\hline $5-8$ & AC control system & $\Delta x_{p}, \Delta x_{q}, \Delta x_{i d}, \Delta x_{i q}$ \\
\hline 9,10 & Measurement filters & $\Delta P_{(m)}, \Delta Q_{(m)}$ \\
\hline 11,12 & $d q$-axis currents & $\Delta i_{t d(m)}, \Delta i_{t q(m)}$ \\
\hline 13,14 & $d q$-axis voltages & $\Delta v_{s d(m)}, \Delta v_{s q(m)}$ \\
\hline 15,16 & DC system & $\Delta v_{d c}, \Delta i_{d c}$ \\
\hline $17-19$ & DC control system & $\Delta x_{c a p}, \Delta x_{c l}, \Delta x_{i d c}$ \\
\hline
\end{tabular}




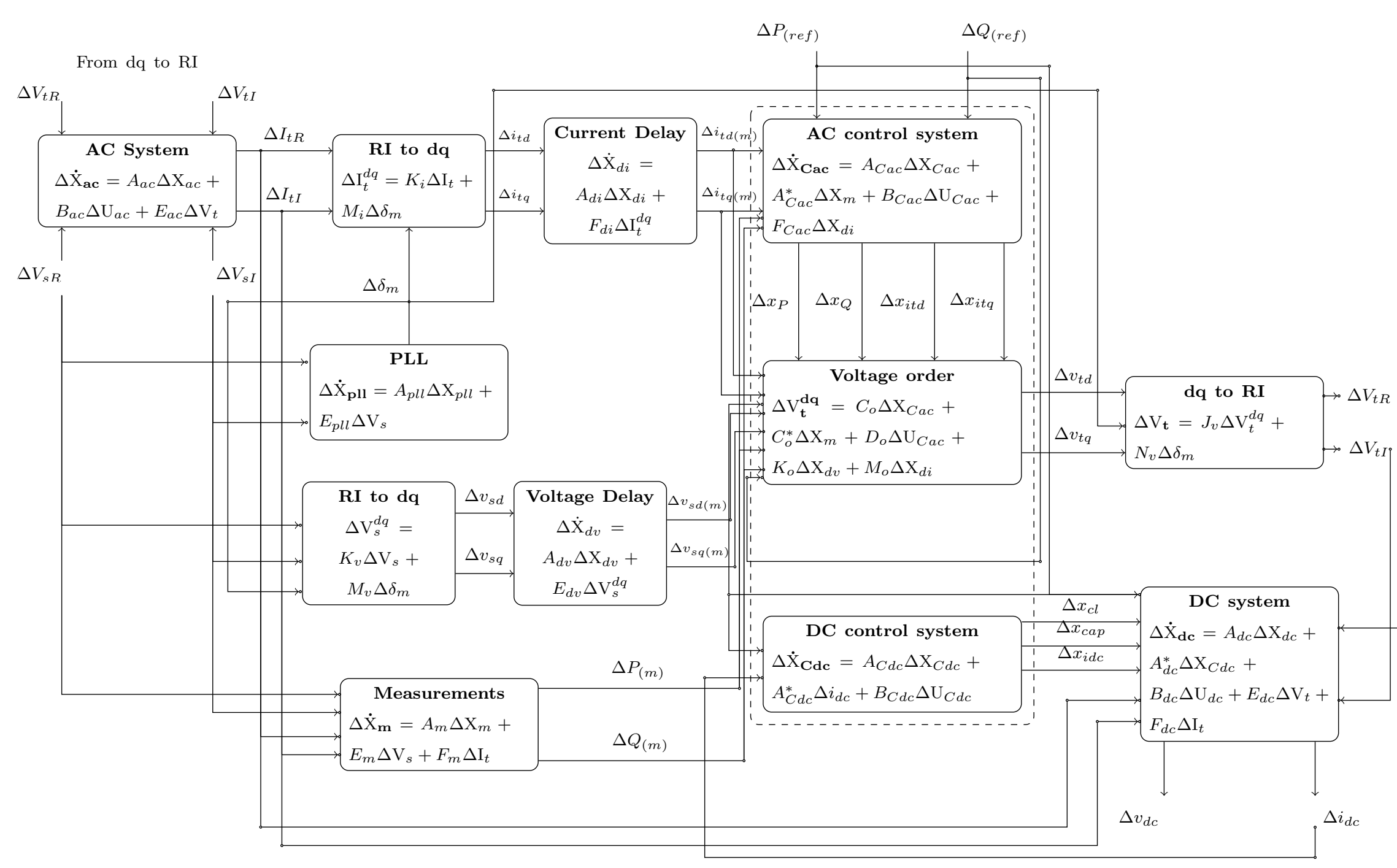

FiguRE 3.9: Modified $19^{\text {th }}$ order small signal model of the hybrid modular multilevel VSC 
TABLE 3.7: Oscillatory modes of the modified state-space model with the converter connected to a moderately strong ac system

\begin{tabular}{c|c|c}
\hline \hline Modes & $\mathrm{f}(\mathrm{Hz})$ & $\zeta(\%)$ \\
\hline Mode 1 & 165.81 & 25 \\
\hline Mode 2 & 103.53 & 28.4 \\
\hline Mode 3 & 58.19 & 68 \\
\hline Mode 4 & 4.38 & 98.5 \\
\hline Mode 5 & 2.64 & 83.3 \\
\hline Mode 6 & 0.76 & 98.6 \\
\hline
\end{tabular}

An eigenvalue analysis was performed on the state matrix of the modified overall state-space model of the hybrid converter for the cases where the converter connected ac system has a moderately high system strength $(\mathrm{SCR}=4.0)$ as well as a weak strength $(\mathrm{SCR}=2.0)$.

The frequency and damping of the oscillatory modes observed with the converter connected to a strong ac system $(\mathrm{SCR}=4.0)$ are given in Table 3.7.

A participation factor analysis performed on the observed oscillatory modes and the results are given in Fig. 3.10.

Modes 3 and 5 were observed with identical frequencies and damping ratios, also in the previous case where the measurement delays for the $d q$ currents and voltages were not included in the small-signal model. Mode 6 in which the states governing the active power controller predominantly participate exhibits a reduction in frequency while the damping remains high. The remaining modes are dominantly contributed by the states of the ac system and the that of the decoupled control scheme which further includes the participation of the states related to the measured currents of the $d$ and $q$ axes. Mode 4 is highly participated by the states associated with the reactive power controller while the states that govern the current controller of the converter are observed to be exciting Mode 2 . 


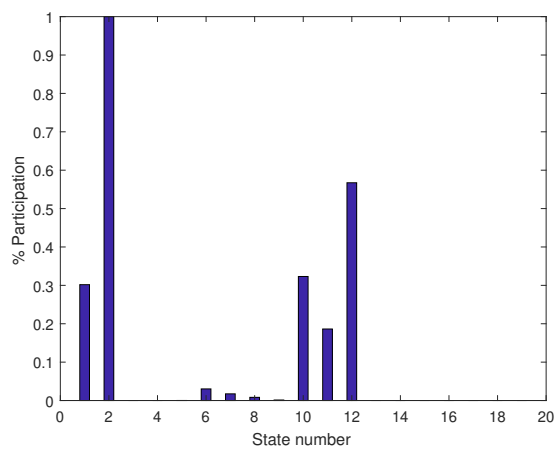

(I) Mode 1

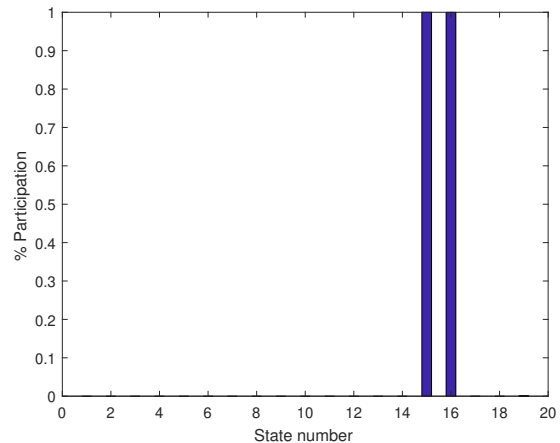

(III) Mode 3

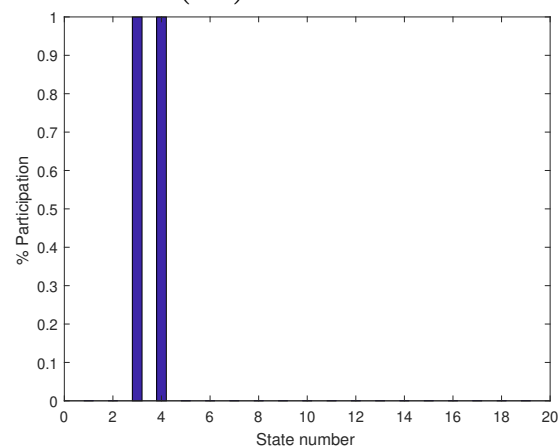

(v) Mode 5

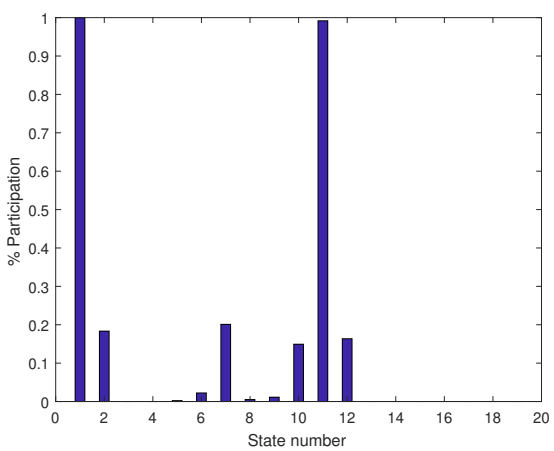

(II) Mode 2

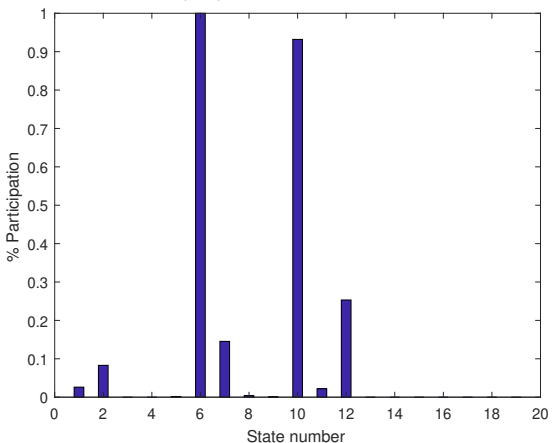

(IV) Mode 4

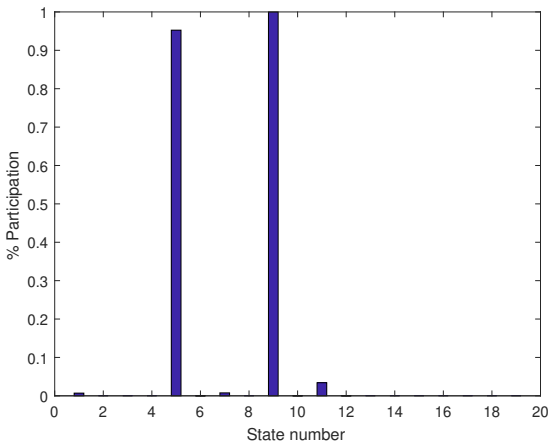

(VI) Mode 6

FiguRE 3.10: Participation factors of the oscillatory mode for $\mathrm{SCR}=4.0$

Similarly the oscillatory modes that are observed when the converter is connected to a weak ac network with $\mathrm{SCR}=2.0$ are given in Table 3.8, while Fig. 3.11 illustrates the results of the participating factor analysis exhibiting the states that highly influence the particular modes under consideration.

Observations made with the converter connected to a weak ac system are similar 
TABLE 3.8: Oscillatory modes of the modified state-space model with the converter connected to a weak ac system

\begin{tabular}{c|c|c}
\hline \hline Modes & $\mathrm{f}(\mathrm{Hz})$ & $\zeta(\%)$ \\
\hline Mode 1 & 107.94 & 42.7 \\
\hline Mode 2 & 63.3 & 31.3 \\
\hline Mode 3 & 58.19 & 68 \\
\hline Mode 4 & 9.26 & 94.8 \\
\hline Mode 5 & 2.64 & 83.3 \\
\hline Mode 6 & 0.7 & 98.9 \\
\hline
\end{tabular}

to that of a converter connected to a network having higher system strength meanwhile states that were observed to be contributing comparatively less in modes 1,2 and 4 exhibit a more significant participation in exciting them in this scenario.

In both these scenarios, the low frequency oscillatory modes are not observed to be highly influenced by the states of the measurement model for the $d q$-axis quantities while the high frequency modes exhibit a significant participation from the $d q$ current components. Low frequency oscillatory modes that were introduced due to the modelling of voltage and current measurement delays are highly damped. Thus it could be concluded that the impact of these delays on the oscillatory stability of the converter is not a concern. Further, the state variables of the $d q$ voltage measurement model have not exhibited a considerable influence on the oscillatory modes of the state-space system. 


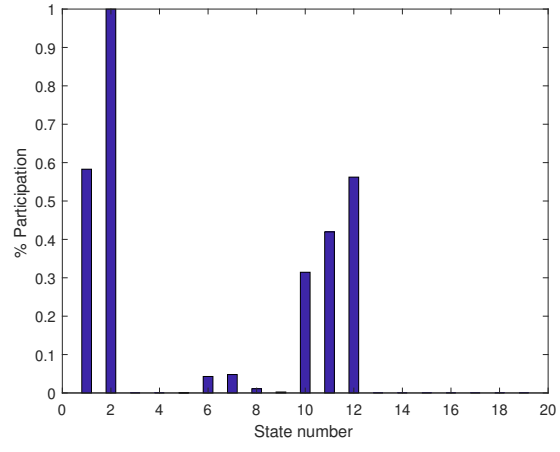

(I) Mode 1

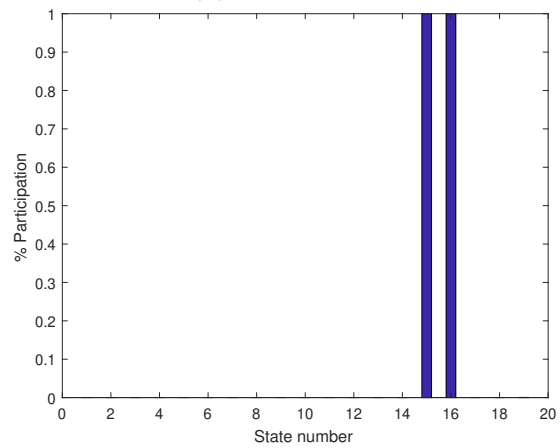

(III) Mode 3

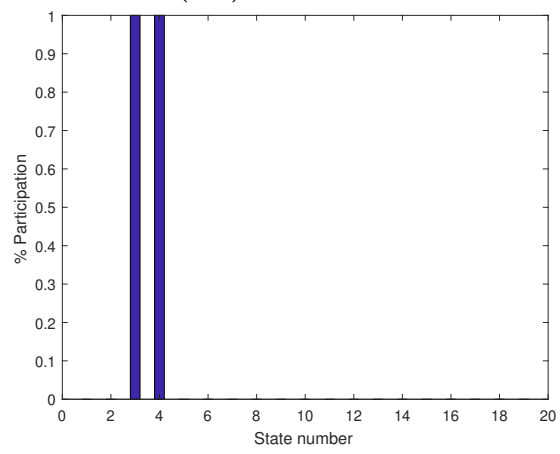

(v) Mode 5

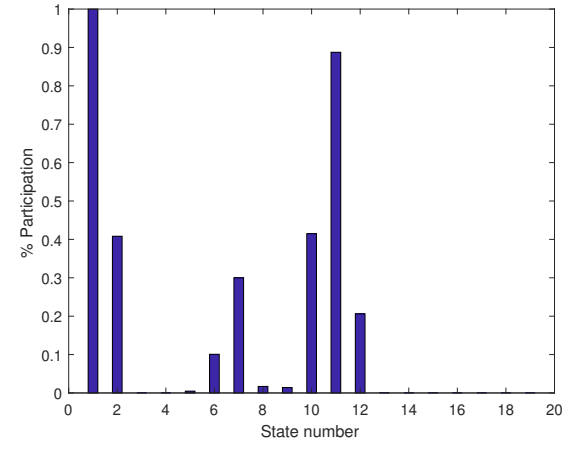

(II) Mode 2

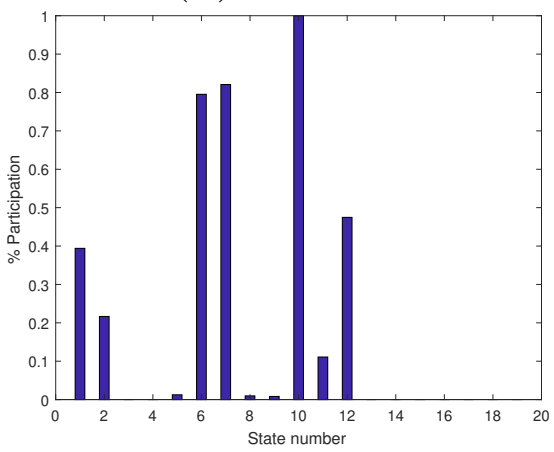

(IV) Mode 4

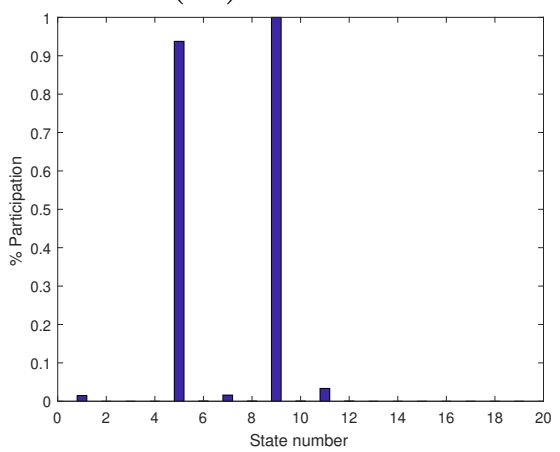

(VI) Mode 6

FiguRE 3.11: Participation factors of the oscillatory mode for $\mathrm{SCR}=2.0$ 


\subsection{Impact of Transformer Saturation on Con- verter Performance with Low SCR}

This section focuses on the results of an analysis based on the observations made in an attempt to validate the developed small-signal model. With the converter connected to an ac network having a low system strength (i.e. $\mathrm{SCR}=2.0$ ), oscillations were observed for steady state operation of the converter in the traces obtained for the measured active and reactive power from the detailed non-linear EMT model developed on PSCAD/EMTDC. Comparison of the traces for the variation in active power $\left(\Delta P_{(a c)}\right)$ obtained from the small-signal model developed in 2.2 and the EMT model is given in Fig. 3.12.

The trace obtained from the detailed EMT simulation contains an oscillation in

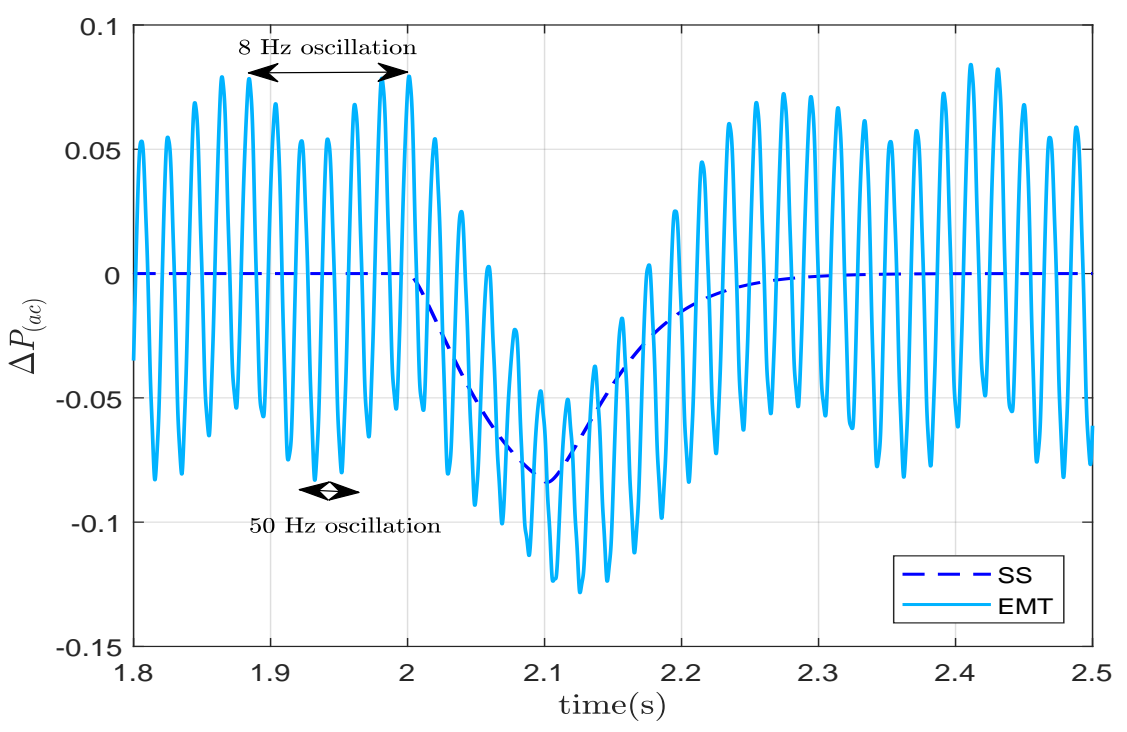

FiguRE 3.12: Variation in active power $\left(\Delta P_{(a c)}\right)$ for a small perturbation in respective reference $P_{(r e f)}$ with the turns ratio of the transformer between ac side to converter side set to $1: 1.1$ 


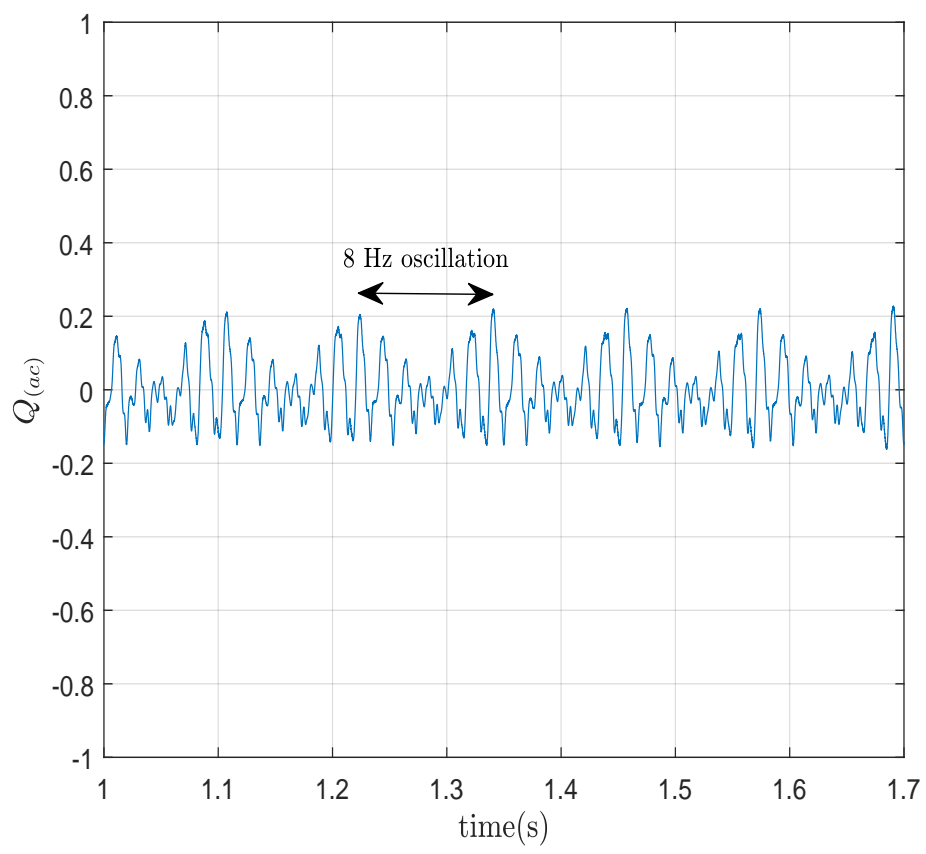

FiguRE 3.13: Low frequency oscillatory component of the variation in reactive power $\left(Q_{(a c)}\right)$ in steady state with the turns ratio of the transformer between ac side to converter side set to 1:1.1

steady state that has a fundamental frequency, $50 \mathrm{~Hz}$ component modulated with a low frequency of $8 \mathrm{~Hz}$ as shown in Fig. 3.12.

A similar observation was made for the variation in reactive power of the network with the converter connected to a weak ac system, delivering $1.0 \mathrm{pu}$ active power and $0 \mathrm{pu}$ reactive power. Under these system conditions, a $50 \mathrm{~Hz}$ oscillation modulated with a low frequency was observed for the steady state trace of reactive power obtained from the EMT simulation. Elimination of the fundamental frequency component from this trace through signal processing resulted in an oscillatory component having a frequency of around $8 \mathrm{~Hz}$ as shown in Fig. 3.13, in a time window of $700 \mathrm{~ms}$.

The ac network has been represented using a dynamic phasor model, which enables investigation of small-signal dynamics in the frequency range of $5-55 \mathrm{~Hz}$ [17]. This 
creates a discrepancy between the two models due to un-modelled dynamics of the converter, hence required further analysis using the detailed non-linear model of the converter.

A Fast Fourier Transform (FFT) on the converter voltage and current waveforms revealed that these quantities consist of undesirably high amounts of $2^{\text {nd }}$ harmonic component. Further it was observed that the modulation index generated by the decoupled $d q$-control scheme, which defines the magnitude of the rectified voltage reference given to the chain-link converters was wobbling around the value for its ceiling limit (i.e. 1.178) specified in 1.3.4.2.

The above two observation led to the fact that the winding voltage on the network side of the ac transformer is insufficient to satisfy the demand of the ac system. The winding voltages were defined by the turns ratio given in Table. 2.1 [4]. The associated controls of the ac system were hence driven to the respective limits as observed from the $M I$, in an attempt to maintain the network voltage at the nominal value which is specified.

In order to rectify this situation which occurs at low system strengths, this thesis proposes to alter the turns ratio of the transformer by changing the tap position when the converter is connected to a weak ac system having a low SCR. Accordingly, the ratios $1: 1$ and 1.1:1, between the ac side and the converter side have been considered in this study.

The winding voltages of the ac transformer modelled in the detailed EMT simulation were changed to obtain the above selected turns ratio. Comparison of traces obtained from the small-signal model and the detailed EMT model with the transformer turns ratio changed to 1:1 and 1.1:1 are given in Fig. 3.14 and Fig. 3.15 respectively. 


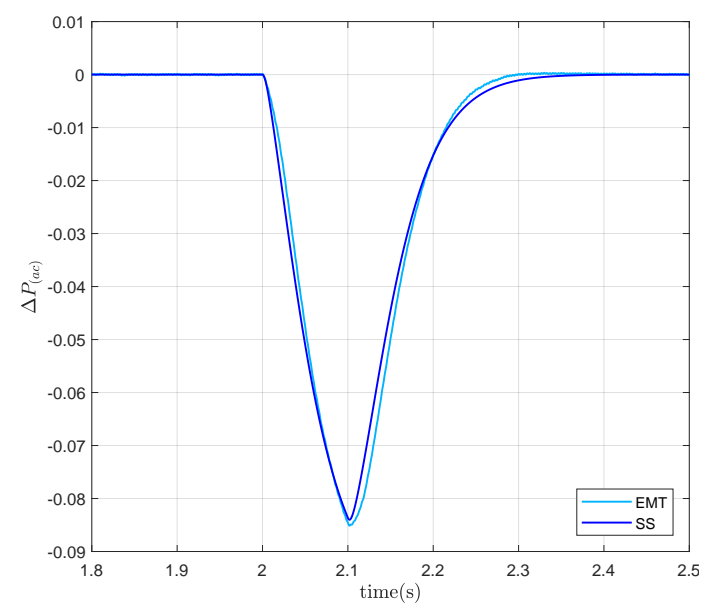

(I)

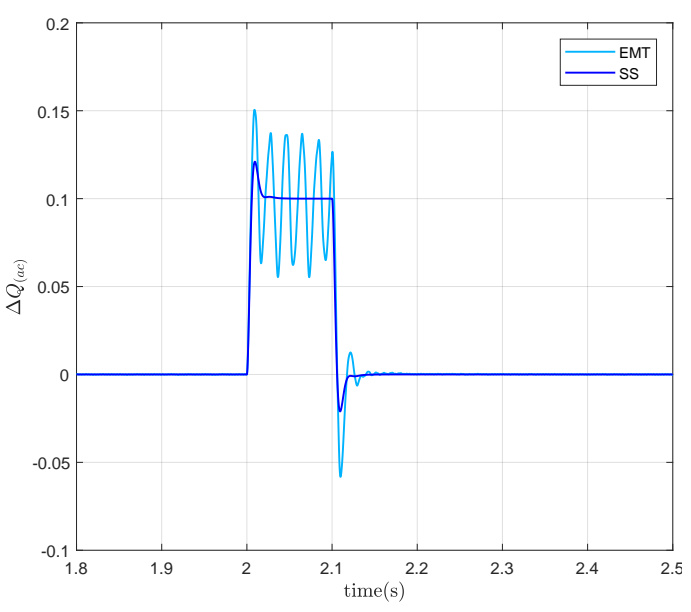

(II)

Figure 3.14: Comparison of results for the turns ratio of the transformer changed to 1:1 between ac side and converter side (I) a small disturbance applied to $P_{(r e f)}$ (II) a small disturbance applied to $Q_{(r e f)}$

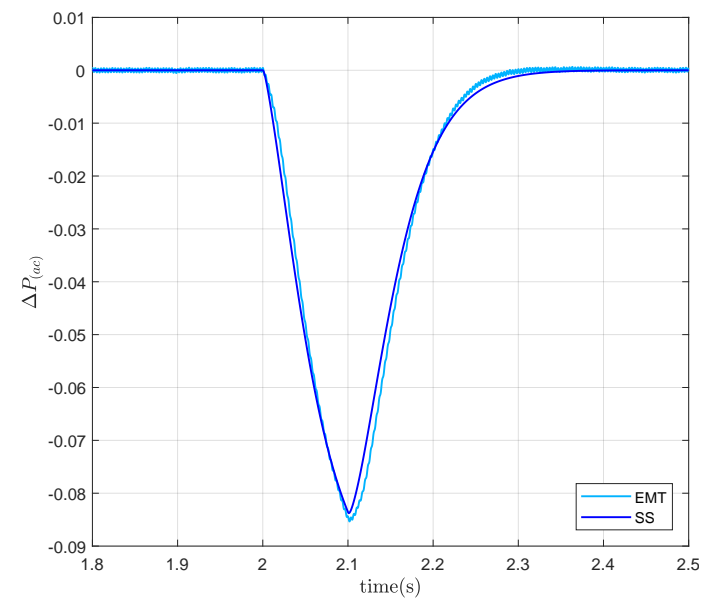

(I)

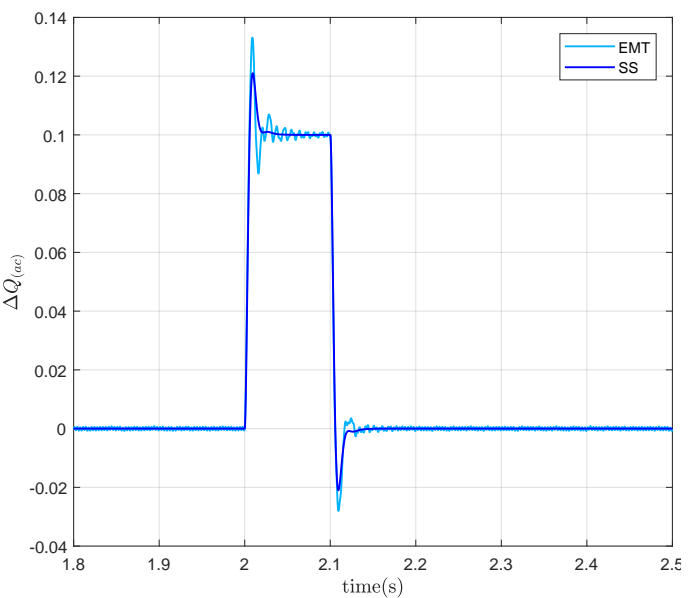

(II)

Figure 3.15: Comparison of results for the turns ratio of the transformer changed to 1.1:1 between ac side and converter side (I) a small disturbance applied to $P_{(r e f)}$ (II) a small disturbance applied to $Q_{(r e f)}$

The latter turns ratio which increases the voltage of the winding connected to the ac network improves the performance of the converter by eliminating low frequency injections. The modulation index generated from the control circuitry is maintained within the specified limits, for the operation of the converter in steady state also during the response to the perturbations applied to control inputs. The 


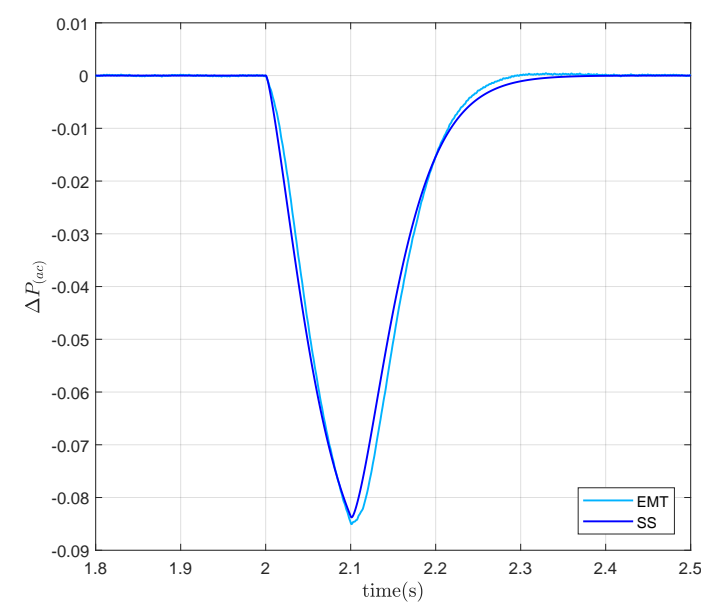

(I)

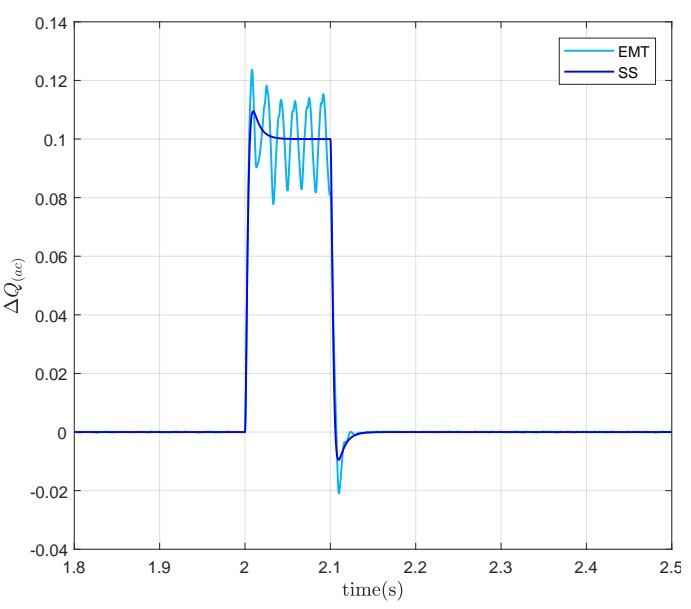

(II)

Figure 3.16: Comparison of results for $\mathrm{SCR}=4.0$ (I) a small disturbance applied to $P_{(r e f)}$ (II) a small disturbance applied to $Q_{(r e f)}$

traces from the developed small-signal model agrees with that of the detailed nonlinear model after the change is being applied to the winding voltages, as given in Fig. 3.15.

Oscillations in steady state operation were not observed in the EMT simulation of the converter connected to strong ac networks, with the parameters set according to Table. 2.1. This is evident from the comparison between traces obtained from the EMT simulation and the developed small-signal model for model validation explained in 2.3, where the SCR of the converter connected ac network is 8.0.

Further the simulation results of the detailed EMT model of the converter connected to a moderately strong ac network having a SCR of 4.0 with a transformer turns ratio specified in Table. 2.1, does not exhibit oscillations for steady state operation as evident from the comparisons given in Fig. 3.16.

The turns ratio for the transformer when connecting the converter to weak ac systems has implications on its performance, as explained in this section. Improper selection of transformer parameters result in low frequency oscillations in system 
quantities including active power and reactive power, hindering the prospective applicability in ac networks with lower system strength.

\section{Chapter Summary}

The eigenvalue analysis performed on the small-signal model developed for the hybrid modular multilevel voltage source converter provides a better insight to its stability, controllability and performance under varying system conditions. Section 3.2.1 has investigated the impact of the system strength of the converter connected ac network on its small-signal stability by selecting two different cases which represent a strong and weak system.

A participation factor analysis enabled the classification of the oscillatory modes. The sensitivity of the oscillatory modes to the variations in the PLL gains and measurement delays of the $d q$-axis currents and voltage quantities have been investigated in this chapter. The converter subjected to a small disturbance does not influence less-damped, low frequency oscillations.

The final section of this chapter emphasizes the importance of an eigenvalue analysis using a developed small-signal model in order to identify the operational issues related to the converter. Observing un-modelled dynamics when comparing the two models for the converter revealed the association of the transformer winding voltages with the low frequency oscillations in the active and reactive power. 


\section{Chapter 4}

\section{Power Transfer Capability of the Hybrid Multilevel Converter}

This chapter evaluates the power transfer capability of the converter, subjected to constraints of the current and voltage ratings. Further, the system strength of the connected ac network could vary depending on its configuration at a given instance, which would impose an additional constraint on the power delivery of the converter.

Power transferred by the converter is analysed for three modes of operation and the voltage, current limitations along with the network constraints have been considered in evaluating the maximum active power deliverable in each scenario. The converter operational modes considered in this study would be with (a) constant reactive power supplied from the converter, (b) constant reactive power absorbed from the connected ac network, and (c) the constant power factor mode of operation in which the reactive power requirement is varied accordingly.

The converter is rated at $20 \mathrm{MVA}$ and for the operation at rated ac voltage of 11 
$\mathrm{kV}$, the maximum steady state current corresponds to $1.05 \mathrm{kA}$. For the operation at a lower voltage than the rated, the maximum current through the converter would be restricted by the ratings of the individual switches corresponding to a value of $1.2 \mathrm{kA}$.

The test system given in Fig. 4.1 is used in this analysis to evaluate the power carrying capability of the converter.

The converter is represented by $V_{c}$ and $\delta$ which refers to the peak phase ac-side voltage and the instantaneous load angle respectively. These parameters for the converter are the outputs of the de-coupled control scheme, generated to cater the active and reactive power requirement of the connected ac network. The converter is connected to an ac network using a transformer having a leakage impedance $X_{t}$ and the voltage at the ac terminal is denoted by $V_{t} \angle \theta$.

For the purpose of evaluating small signal stability of the converter, the transformer has been considered ideal in 2.2.1. However, for the evaluation of practical limits for power transfer capability of the converter a finite transformer leakage reactance has been taken into account thereby replicating a practical scenario which provides a realistic insight to the operational constraints of the hybrid multilevel VSC topology. This study uses a $15 \%$ reactance $(=0.15 \mathrm{pu})$ on the transformer MVA base. A Thevenin equivalence comprising of a voltage source $V_{s}$ and a series

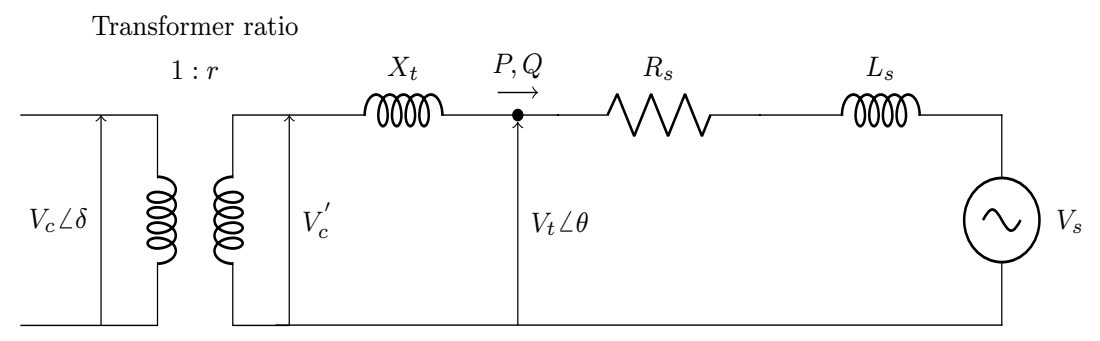

FiguRE 4.1: Schematic of the test system for the hybrid modular multilevel VSC 
RL branch whose values are selected in order to model strong and weak ac systems represents the converter connected ac network.

The active power requirement $(P)$ and reactive power demand $(Q)$ are determined by the connected ac network while the voltage at the infinite bus $\left(V_{s}\right)$ is maintained at $1.0 \mathrm{pu}$.

\subsection{Calculation of the active power delivered by the converter}

Given the values for $P$ and $Q$, the Gauss-Siedel method for power flow solutions is used to recursively calculate the ac terminal voltage $\vec{V}_{t}$ using Equation (4.1).

$$
\vec{V}_{t}=\frac{1}{Y_{22}}\left[\frac{P-j Q}{\vec{V}_{2}^{*}}-Y_{21} \vec{V}_{s}\right]
$$

$\vec{X}$ denotes the vector form of the variable $X$.

$$
\begin{aligned}
& Y_{22}=\frac{1}{\left(R_{s}+j \omega L_{s}\right)} \\
& Y_{21}=-Y_{22}
\end{aligned}
$$

The voltage at the ac terminal of the transformer $\vec{V}_{c}^{\prime}$ is obtained from Equation (4.2) by adding the voltage drop due to the leakage impedance of the transformer.

$$
\vec{V}_{c}^{\prime}=\vec{V}_{t}+j X_{t}\left(Y_{21} \vec{V}_{s}+Y_{22} \vec{V}_{t}\right)
$$


For a transformer winding voltage ratio of 1:r $(\mathrm{r}=1.1)$ between the converter side and the ac side, the converter voltage $\vec{V}_{c}$ could be obtained from Equation (4.3).

$$
\vec{V}_{c}=\frac{\vec{V}_{c}^{\prime}}{r}
$$

\subsection{Constraints imposed on maximum power de- liverable by the converter}

This section elaborates an investigation of the power handling capability of the converter based on a power flow analysis performed using the test system given in Fig. 4.1. It was further observed that the maximum power delivered by the converter is restricted by the below stated constraints.

(a) Limitation of the maximum current through the converter

(b) Limitation of the converter voltage corresponding to the configuration and operation

(c) Impact of the system strength, parametrized by the SCR

A similar study is presented in [23] where the impact of the Volt-Ampere rating of the converter and the network strength have been considered. The analysis presented in this section considers the converter voltage limits in addition to the above constraints in evaluation of the maximum power delivered by the converter.

The three constraints identified above are further explained in section 4.2.1, 4.2.2 and 4.2 .3 respectively. 


\subsubsection{Limits imposed by the maximum current through the converter}

The maximum current through the converter is limited either by the current rating for the switches or its MVA rating for continuous operation. The minimum of the two defines the maximum permissible current through the VSC, which limits the maximum active power deliverable by the converter.

The test system of the hybrid modular multilevel converter utilized for the underlying analysis is rated for 20 MVA, which corresponds to a converter current of $1.05 \mathrm{kA}$, while the IGBTs that are used for the converter has a current carrying capability of $1.2 \mathrm{kA}$. Thus the maximum allowable current through the converter is determined by the lowest of the two which corresponds to the MVA rating of the converter.

For example if the converter is operating at the rated voltage, the maximum current according to the MVA rating is $I_{\max , M V A}=1.05 \mathrm{kA}$. If the converter is operating at $0.8 \mathrm{pu}, I_{\max , M V A}=1.3 \mathrm{kA}$ which is greater than the rated current of $1.2 \mathrm{kA}$ for the IGBTs. Hence, the maximum current is $1.2 \mathrm{kA}$, not $1.3 \mathrm{kA}$.

\subsubsection{Limits imposed by the converter voltage}

The peak phase voltage generated by the converter controls is bounded by the limits for the Modulation Index (MI) in multilevel converter configurations which utilize multilevel modulation strategies for the wave-shaping function. The injection of third harmonic voltage further restricts the range for the MI of the given converter topology compared to a conventional VSC. 
These restrictions were described in detail in Chapter 1 as summarised by equations (1.13), (1.14) and (1.15). These equations are reproduced here as Equation (4.4)-(4.6) for the convenience of the reader.

$$
\begin{gathered}
M I=\frac{2 \pi}{6\left(1+\alpha_{3} / 3\right)} \\
-1 / 3 \leq \alpha_{3} \leq 1 \\
0.785 \leq M I \leq 1.178
\end{gathered}
$$

The ratio between the peak phase ac voltage and half of the dc-link voltage define the MI for a two-level VSC. Hence the peak phase voltage magnitude $V_{c}$ of the converter is constrained to the range given in Equation (4.7).

$$
0.785 \times \frac{V_{G}}{2} \leq V_{c} \leq 1.178 \times \frac{V_{G}}{2}
$$

where $V_{G}$ denotes the dc-link voltage of the converter.

The range for active power transfer is thus constrained by the limits for the converter voltage.

\subsubsection{Impact of the system strength on the power carrying capability of the converter}

The study has been conducted on a converter connected to strong $(\mathrm{SCR}=4.0)$ and weak $(\mathrm{SCR}=2.0)$ ac networks which analyses the impact of the system strength on the active power transfer of the converter. 


\subsection{Evaluation of power handling capability for different mode of converter operation}

Sections 4.3.1, 4.3.2 and 4.3.3 analyse the maximum active power $(P)$ delivered by the converter for below given modes of operations respectively.

(a) Converter operation with reactive power supplied from the converter itself

(b) Converter operation with reactive power supplied from the connected ac network

(c) Converter operation at constant power factor.

The test system given in Fig. 4.1 and system parameters given in Table 4.1 have been used for this study. For each of these test scenarios, the limits for the converter current, voltage and the impact of the SCR have been incorporated to obtain the maximum power deliverable.

\subsubsection{Power handling capability of the converter with con- stant reactive power supply}

Maximum active power $(P)$ delivered by the converter for different levels of constant reactive power supply $(Q)$ in the range of 0 to $0.3 \mathrm{pu}$ is calculated.

For values of $P$ in the range of $0-20 \mathrm{MW}$, the variation of the terminal voltage $V_{t}$ with active power delivered by the converter is observed at different levels of $Q$ supplied in the range of 0-6 MVAr $(0-0.3 \mathrm{pu})$. The results are plotted on a per unit scale and presented for strong and weak ac network conditions in Fig. 4.2 and Fig. 4.3 respectively. The dotted lines represent the permissible limits $( \pm 5 \%)$ for the variation of the terminal voltage $V_{t}$ from the rated value. 
TABLE 4.1: Test system parameters

\begin{tabular}{l|c|c}
\hline \hline \multicolumn{1}{c|}{ System Parameter } \\
\hline Rated active power $P_{\text {rated }}$ & \multicolumn{2}{|c}{$20 \mathrm{MW}$} \\
AC grid voltage $V_{s}$ (L-L RMS) & \multicolumn{2}{|c}{$11 \mathrm{kV}$} \\
DC grid voltage $V_{G}$ & \multicolumn{2}{|c}{$20 \mathrm{kV}$} \\
AC grid frequency $f$ & \multicolumn{2}{|c}{$50 \mathrm{~Hz}$} \\
Transformer ratio (ac side:converter side) & \multicolumn{2}{|c}{$1.1: 1$} \\
Transformer leakage reactance $X_{t}$ & \multicolumn{2}{|c}{$0.15 \mathrm{pu}$} \\
\hline AC system impedance & $\mathrm{SCR}=4.0$ & $\mathrm{SCR}=2.0$ \\
\hline$R_{s}$ & $20.29 \mathrm{~m} \Omega$ & $42.83 \mathrm{~m} \Omega$ \\
$L_{s}$ & $4.5 \mathrm{mH}$ & $9.5 \mathrm{mH}$ \\
\hline
\end{tabular}

In Fig. 4.2, end point on each curve (denoted by ' $X_{n}$ ') corresponds to the maximum active power delivered, limited by the Volt-Ampere rating thereby the current through the converter reaching $1.0 \mathrm{pu}$.

In the case with Fig. 4.3, the end point of the first curve (marked as ' $Y_{1}$ ') corresponds to the value of active power where both the current and voltage limits for the converter have been reached. The rest of the curves have an end point (' $Y_{n}$ ') that corresponds to the rated current operation of the converter.

\section{Validation of the analytical calculations against simulation results}

Results obtained through the analytical calculation were validated against the detailed, non-linear EMT simulation model of the hybrid modular multilevel converter developed on PSCAD/EMTDC with the parameters given in Table 4.1. The theoretical maximum active power transfer of the converter for each value of $Q$ considered, is compared against the actual active power transfer observed from the simulation case of the converter. The comparison is given in Fig. 4.4.

Each point denoted as analytical in Fig. 4.4 represents the theoretical maximum power which corresponds to the end points of the curves in Fig. 4.2 and Fig. 4.3. 


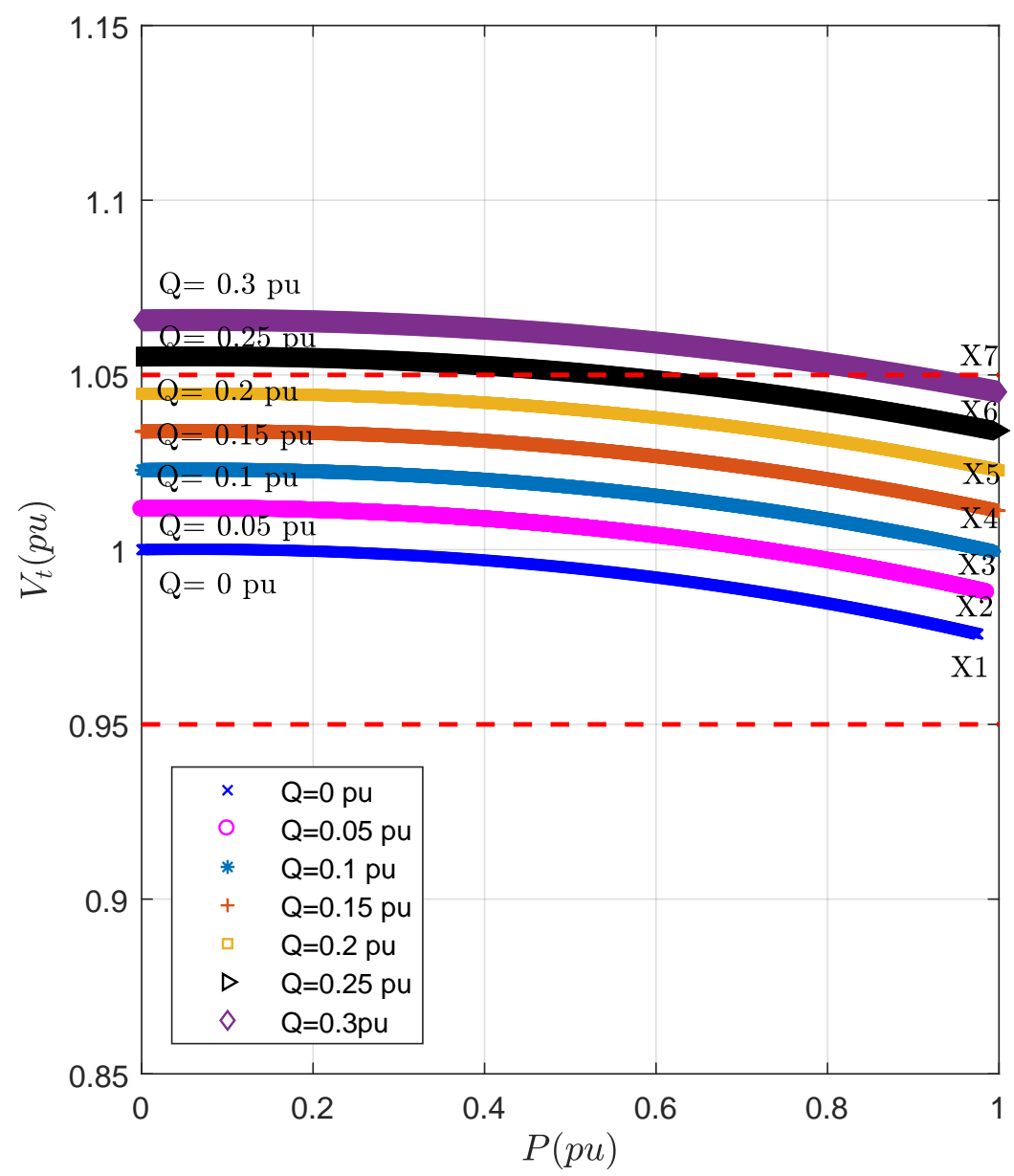

FiguRE 4.2: Variation of $V_{t}$ with active power delivered for a converter connected to a strong ac network with $\mathrm{SCR}=4.0$; constant $Q$ operation

The developed converter model on PSCAD/EMTDC was observed to be dynamically unstable for values of reactive power supply beyond 5 MVAr $(0.25 \mathrm{pu})$ while connected to a weak ac network. Thus validation of results are presented to this limit of reactive power supply, $\mathrm{Q}<5 \operatorname{MVAr}(\mathrm{Q}<0.25 \mathrm{pu})$ for $\mathrm{SCR}=2.0$.

The analysis presented in 4.3.1 leads to the following two conclusions;

- For each value of $Q$, the terminal voltage decreases with the increase of active power delivered by the converter. Thus a minimum reactive power requirement exists to deliver $1.0 \mathrm{pu}$ active power while maintaining the terminal 


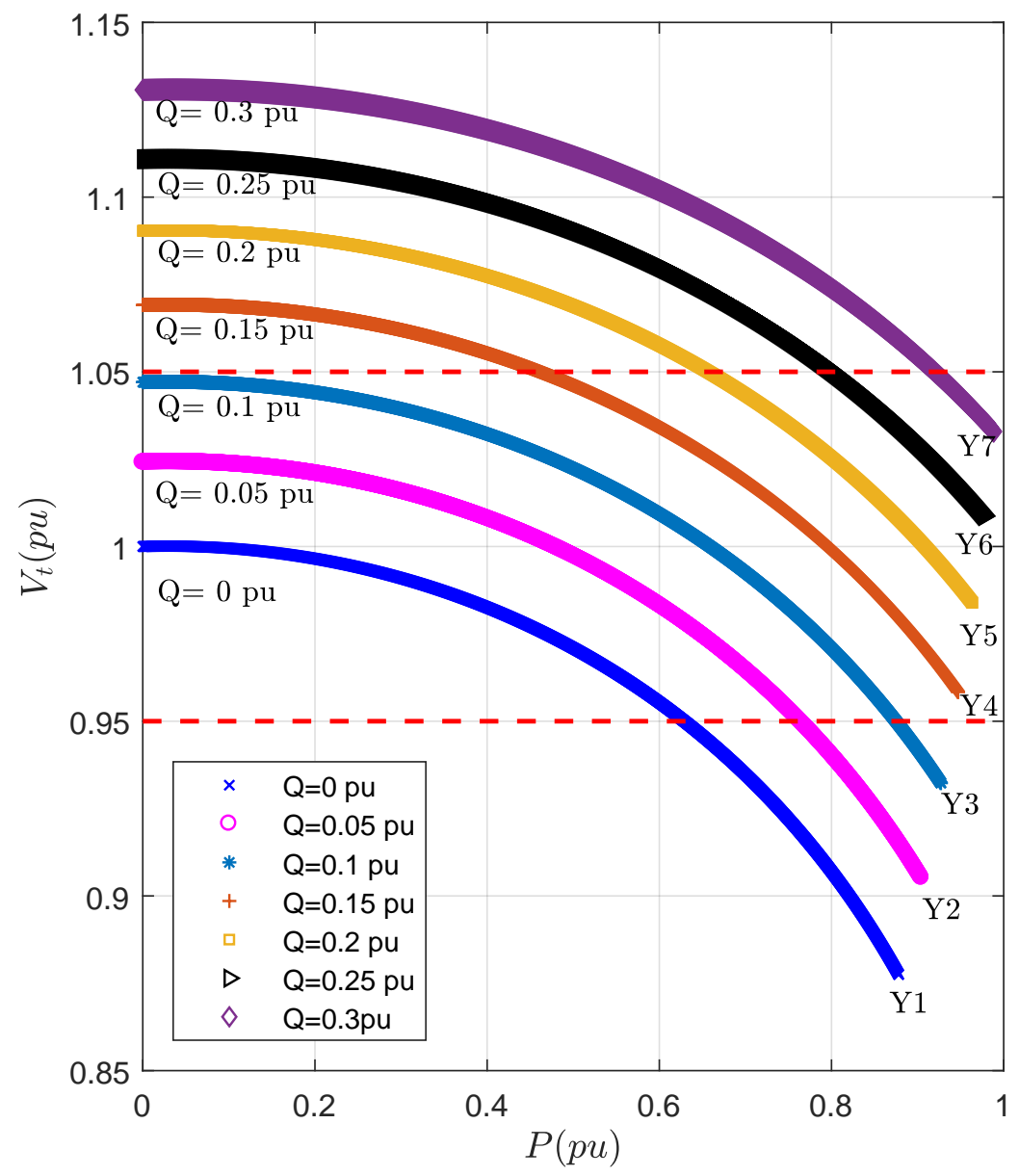

FiguRE 4.3: Variation of $V_{t}$ with active power delivered for a converter connected to a weak ac network with $\mathrm{SCR}=2.0$; constant $Q$ operation

voltage $V_{t}$ within its permissible range for continuous operation.

- The theoretical maximum power obtained from the steady state analysis closely matches that of the dynamic simulation which signifies the proper tuning of the control system of the converter by selecting appropriate values for the gains of PI controllers. 


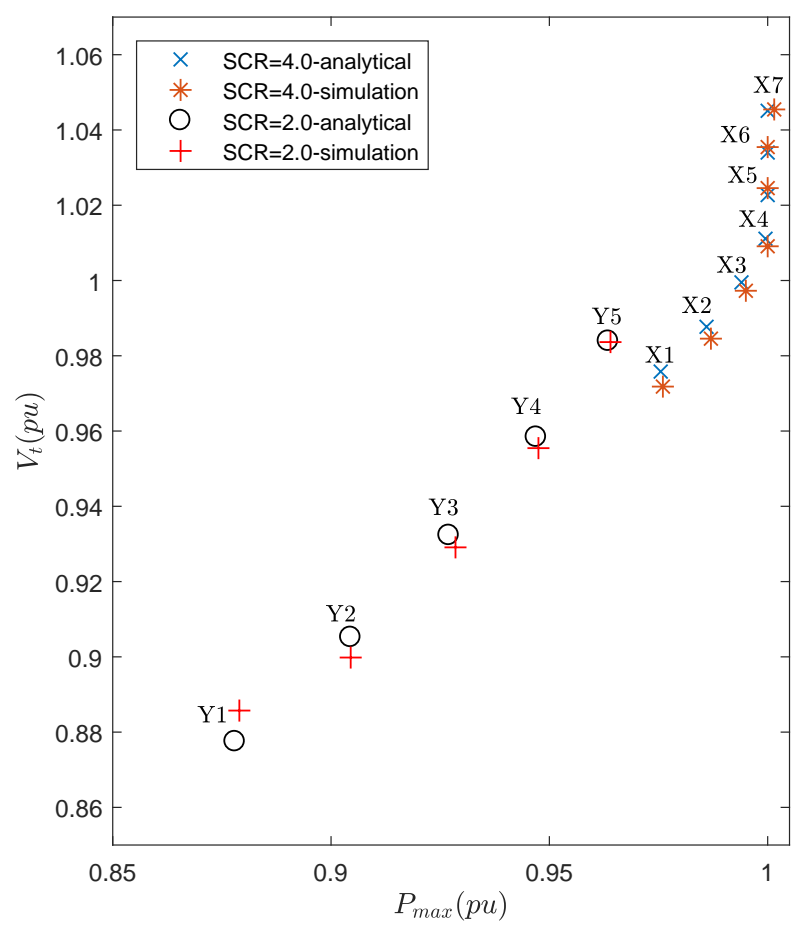

Figure 4.4: Comparison of results for $P_{\max }$

\subsubsection{Power handling capability with reactive power ab- sorbed by the converter}

Variation of the terminal voltage for active power delivery, with the converter absorbing reactive power (negative $Q$ ) has been analysed and Fig. 4.5 and Fig. 4.6 illustrate the results for the system strengths of SCRs 4.0 and 2.0 respectively.

The terminal voltage at the maximum power delivered by the converter reduces as the reactive power absorption from the grid increases. The active power represented by 'A' corresponds to the maximum power the converter is capable of handling while maintaining current at its upper limit. Point 'B' on the curve corresponds to the minimum active power that is required to be supplied by the converter in order to maintain its voltage above the lower limit, as given from Equation (4.7). 


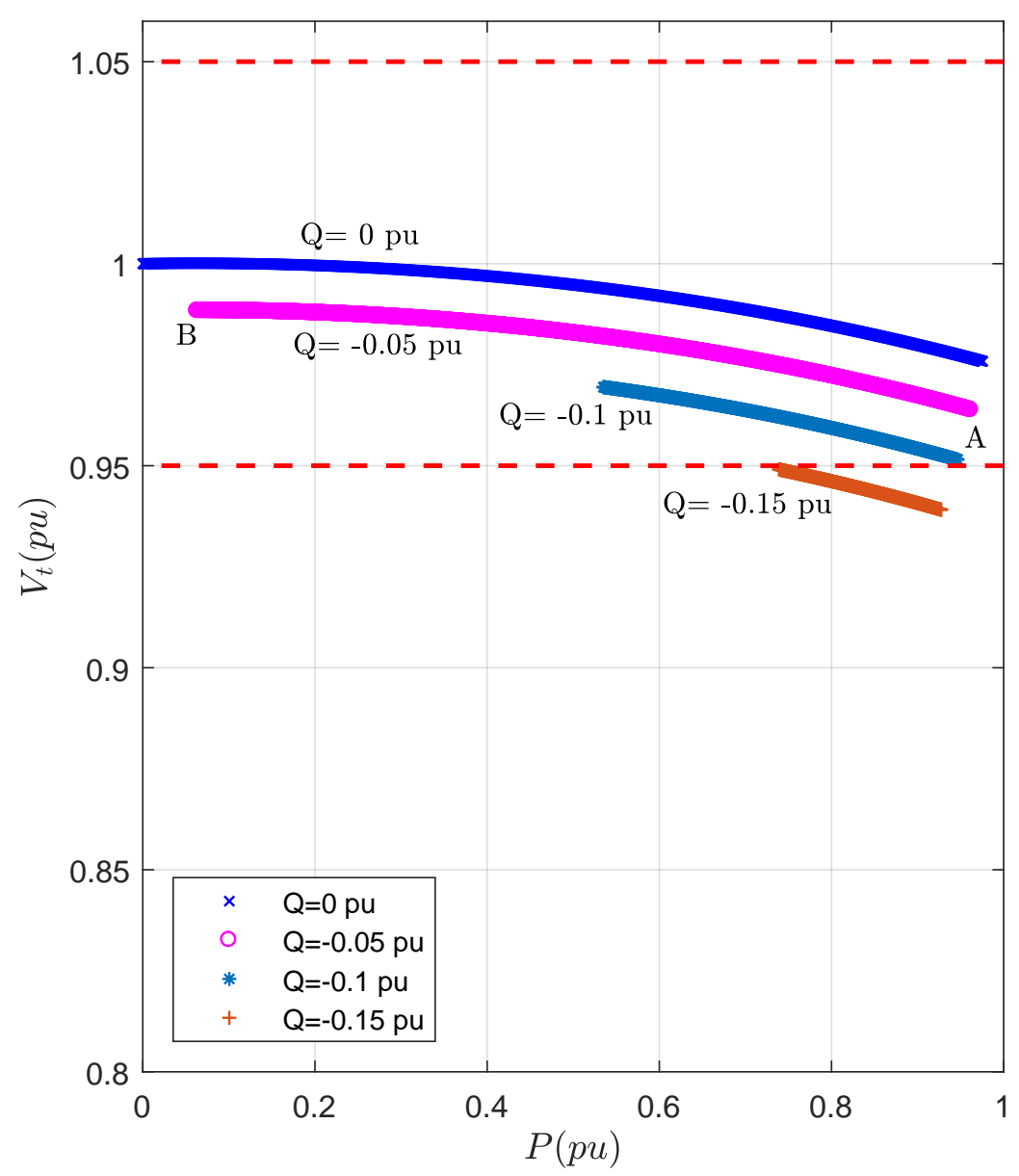

FiguRE 4.5: Variation of $V_{t}$ with active power delivered for a converter connected to a strong ac network with $\mathrm{SCR}=4.0 ; Q$ absorbed from the network

The analysis presented in 4.3.2 leads to the following conclusions;

- The minimum limit for the converter voltage imposes a constraint on the power handling capability of the converter where a minimum required value of active power was observed

- The converter connected to a weak ac network is not suitable for operation with the reactive power absorbed from the network. 


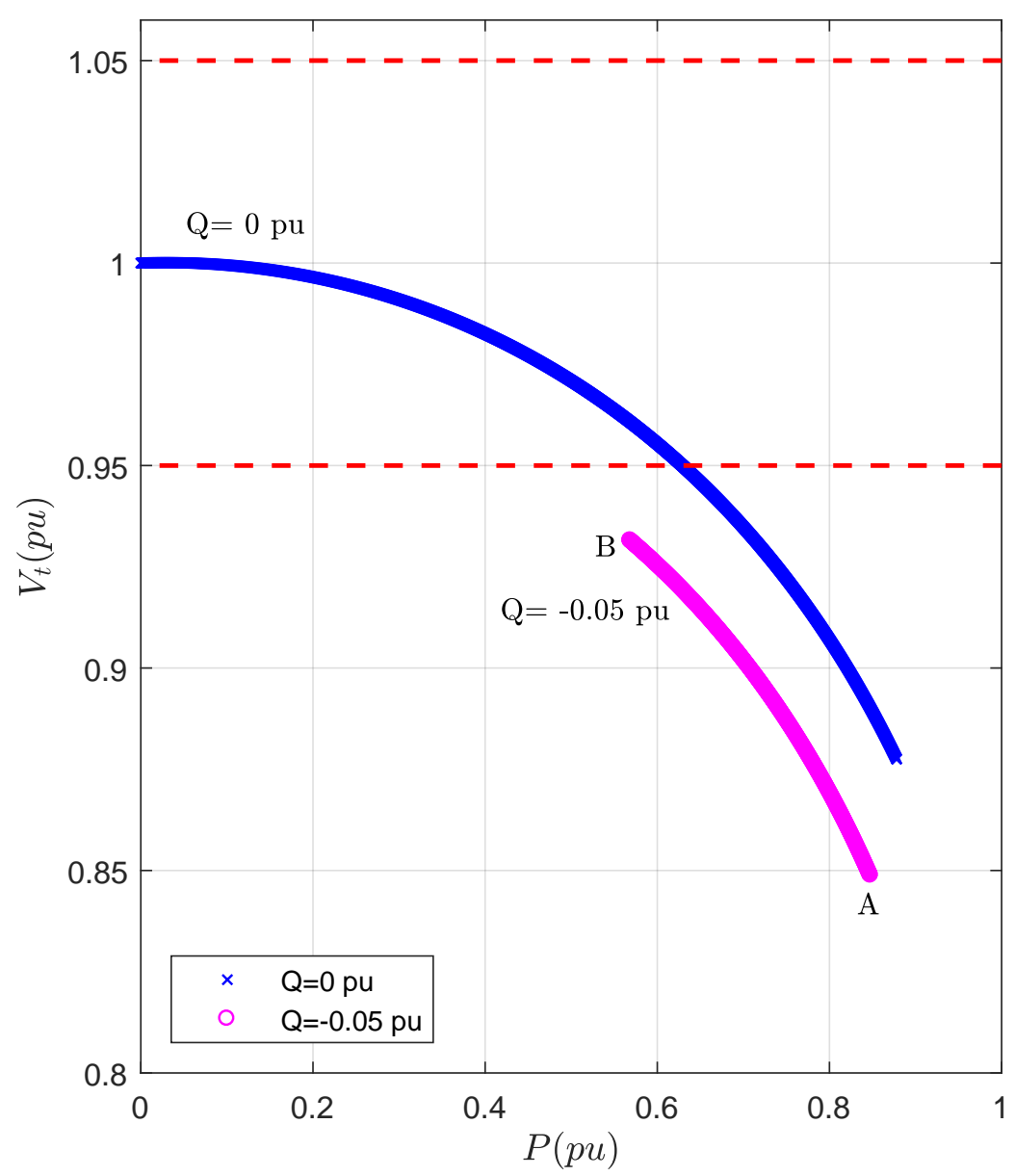

FiguRE 4.6: Variation of $V_{t}$ with active power delivered for a converter connected to a weak ac network with $\mathrm{SCR}=2.0 ; Q$ absorbed from the network

\subsubsection{Power handling capability of the converter with con- stant power factor operation}

An analysis similar to the previous study was conducted in which the power delivery of the converter with a constant power factor has been evaluated. The variation of the ac terminal voltage $V_{t}$ with increase of active power delivered by the converter from $0 \mathrm{MW}$ to $20 \mathrm{MW}$ (rated power) was analysed for strong and weak ac systems of the converter. The theoretical maximum for active power transfer of 


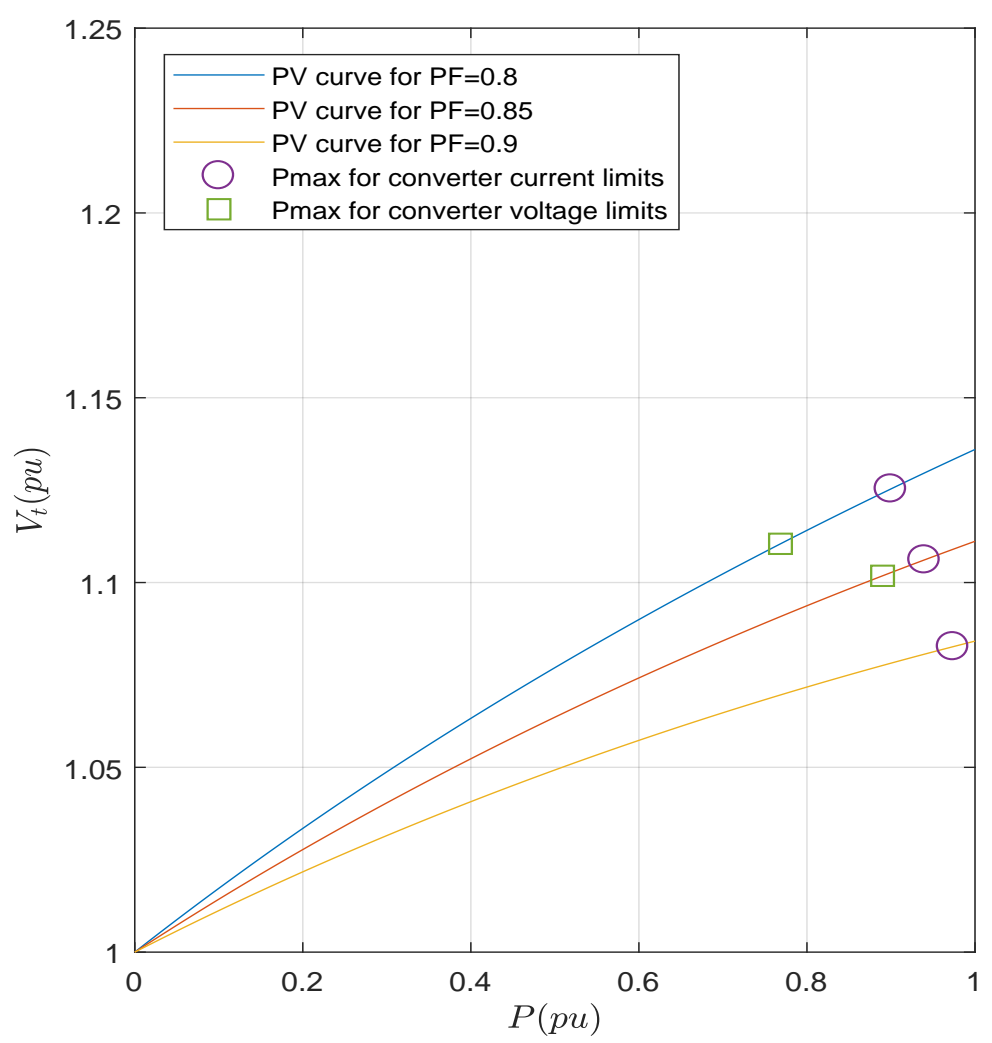

FiguRE 4.7: Variation of $V_{t}$ with active power delivered for a converter connected to a strong ac network with $\mathrm{SCR}=4.0$; constant $\mathrm{PF}$ operation

the converter corresponding to the converter current and voltage restraints have been identified. The power-voltage curves for the converter connected to a strong ac network with $\mathrm{SCR}=4.0$ and a weak system of $\mathrm{SCR}=2.0$. are given in Fig. 4.7 and Fig. 4.8 respectively. The terminal voltage is observed to be increasing as the converter delivers more active power, at constant power factor. This is mainly due to the converter compensating the reactive power requirement to facilitate the active power supply.

Squares on the curves refer to the maximum power delivery that is restricted by the voltage limit for the converter, dictated by the permissible range of the MI of the converter while the circles correspond to the upper limit for power transfer that is constrained by the maximum current that is allowed to flow through the converter. 


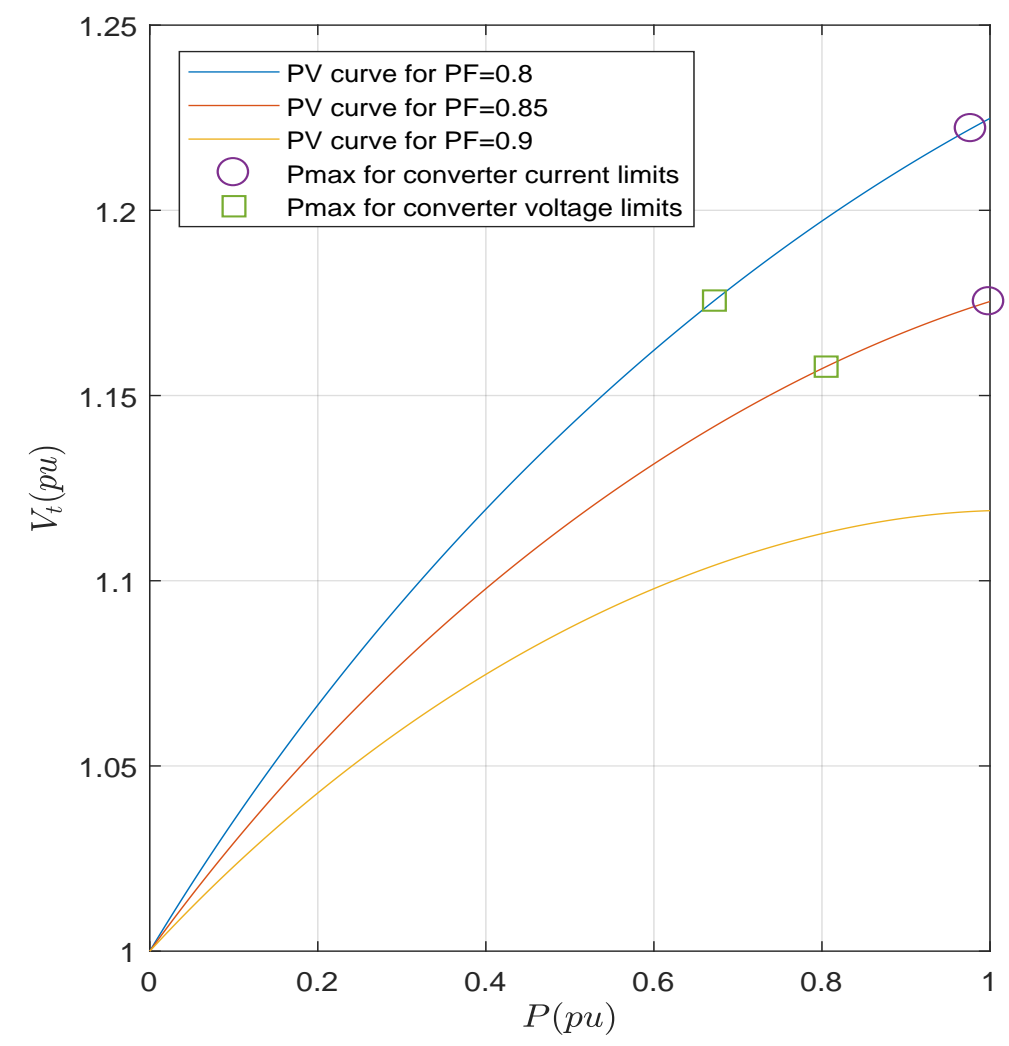

FiguRE 4.8: Variation of $V_{t}$ with active power delivered for a converter connected to a weak ac network with $\mathrm{SCR}=2.0$; constant $\mathrm{PF}$ operation

However the power handling capability of the converter for this particular mode of operation will further be limited by the permissible range for the terminal voltage.

The analysis presented in 4.3.3 leads to the following conclusion;

- Power carrying capability of the converter could be improved through increasing the power factor of the system thereby limiting the rise in terminal voltage to be within the allowable range for overvoltage as per the grid code. The maximum active power delivered would correspond to the value at which the converter current reaches its maximum. 


\section{Chapter Summary}

Maximum power carrying capability of the novel hybrid modular multilevel VSC is restrained by the maximum current through the converter and the permissible range for the converter voltage magnitude. The power handling capability of the converter varying between 0 and the rated power has been evaluated in the range of $-0.3 \mathrm{pu}$ to $+0.3 \mathrm{pu}$ of reactive power supply. The terminal voltage at maximum power delivered could be improved by supplying more reactive power. The tuned PI controllers have enabled the converter to achieve the theoretical maximum active power speculated by the analytical power flow solved in this section.

The impact of the system strength of the connected ac network has been investigated and the converter is not suitable to be operated with reactive power absorbing from the network, when the strength of the system changes to a low value.

Improving the power factor of the system for constant power factor mode of operation for the converter enables more active power to be transferred while maintaining the terminal voltage within the acceptable limits. 


\section{Chapter 5}

\section{Conclusions, Contributions and}

\section{Future Work}

\subsection{Conclusions}

The primary focus of this thesis was to develop a small-signal model for a novel Hybrid Modular Multilevel Voltage Source Converter topology. A detailed eigenvalue analysis has been performed on the state-space model obtained in order to evaluate the stability constraints of the converter while operating under different system conditions.

The second objective was to analyse the power handling capability of this converter configuration, bounded by the limitations of its own as well as the system constraints.

Given below in point form are the general conclusions that could be arrived at, through the detailed analysis presented in this thesis. 
- The linearized state-space system accurately models the dynamics of the ac system, dc system, control circuitry, measurement filters and the PLL of the converter where the validation exhibits close correspondence to the results obtained through a detailed, non-linear EMT simulation model.

- The novel Hybrid Modular Multilevel VSC configuration does not exhibit instabilities due to low-frequency oscillations while the control inputs given to the system have been subjected to small perturbations; under both scenarios with the converter being connected to strong and weak ac networks. However, the transformer winding voltage ratio improvises adverse effects on the steady-state performance of the converter due to low-frequency oscillations observed in system quantities such as active power and reactive power, caused by saturation of the transformer. Hence this thesis proposes to have a higher voltage on the transformer winding connected to the ac side to mitigate injection of low-frequency oscillations to the system.

- The oscillatory stability of the converter is not affected by the variation in the PLL gains and measurement delays associated with the $d q$-axis currents and voltages since the low-frequency modes remain highly damped.

- The power carrying capability of the converter with reactive power supplied is predominantly restricted by the maximum current through it. The terminal voltage at any given value for active power transfer could be increased, restricted to the permissible range for continuous operation, by increasing the reactive power supplied by the converter. Furthermore the limits set on the modulation index, by the restricted range for $3^{\text {rd }}$ harmonic voltage injection to maintain a constant voltage across the dc-link of the converter constraints the allowable range of active power transfer of the converter while absorbing reactive power from the network. This analysis further revealed 
that this converter configuration is not capable of delivering useful power to the connected ac system while absorbing reactive power from the network.

\subsection{Contributions}

The main contributions of the thesis are summarized below;

- Development of a linearized small-signal model for the novel hybrid modular multilevel VSC topology which could be utilized for stability evaluation studies performed on power systems based on HVDC transmission.

- Stability assessment of the converter, connected to ac networks having different system strengths and analysis of sensitivities to changes in controller dynamics (i.e. PLL gains and delays associated with measurement of voltages and current).

- Important guidelines to set winding voltage ratio for the ac transformer in order to mitigate low-frequency oscillations injected to the system due to saturation of the transformer voltage thereby the control circuitry.

- Analysis of the power carrying capability of the converter connected to strong and weak ac networks, operating with reactive power supplied by the converter and absorbed from the network.

The key contributions of this thesis have been published in the following reputed conference proceedings. 


\section{Conference Papers}

1. C. Nanayakkara Yapa, U.D. Annakkage, S. Filizadeh, "Stability Analysis of a Hybrid Modular Multilevel Voltage Source Converter", presented at IEEE Canada Electrical Power and Energy Conference (EPEC 2018), Toronto, ON, Canada, Oct. 2018.

2. C.A. Nanayakkara Yapa, U.D. Annakkage, S. Filizadeh, C. Karawita "Analysis of maximum power transfer capability of a hybrid modular multilevel converter", accepted for the $15^{\text {th }}$ International conference on $A C$ and $D C$ power transmission (ACDC 2019), Coventry, UK, Feb. 2019.

\subsection{Future Works}

Based on the experience gained during the work presented in this thesis and depending upon the growing interest on hybrid multilevel voltage source converters to expand the transmission system based on HVDC by reaching better voltage and power quality levels at a reduced converter footprint and losses; a list of possible future directions of this work are outlined below.

- Extensive research being carried out on developing multi-terminal DC networks using VSCs, privileged by the converter configuration provides practical applications for the hybrid converter topology discussed in this thesis, which is a prospective candidate for HVDC transmission. Stability assessment studies on such power systems using small-signal modelling techniques are pre-requisites prior to implementation. Thus the developed model presented in this thesis could be incorporated with the available small-signal 
modelling tools for stability evaluation of the connected large power system for small perturbations in system quantities.

- The gains of the ac and dc system controllers associated with the converter were obtained in this thesis via heuristic methods where as utilization of frequency domain controller tuning procedures proposed in research for simultaneous tuning of converter controls would be efficient, resulting in better performance at the same time. These novel approaches could be incorporated with the developed models for the converter to enhance the performance while subjected to small disturbances. 


\section{Bibliography}

[1] Michael M.C. Merlin, Tim C. Green, Paul D. Mitcheson, David R. Trainer, Roger Critchley, Will Crookes, and Fainan Hassan. The alternate arm converter: A new hybrid multilevel converter with DC-fault blocking capability. IEEE Trans. Power Deliv., 29(1):310-317, 2014.

[2] M.M.C. Merlin, T.C. Green, P.D. Mitcheson, D.R. Trainer, D.R. Critchley, and R.W. Crookes. A new hybrid multi-level voltage-source converter with DC fault blocking capability. 9th IET Int. Conf. AC DC Power Transm. (ACDC 2010), pages 056-056, 2010.

[3] Grain Philip Adam, Khaled H. Ahmed, Stephen J. Finney, Keith Bell, and Barry W. Williams. New breed of network fault-tolerant voltage-sourceconverter HVDC transmission system. IEEE Trans. Power Syst., 28(1):335$345,2013$.

[4] R. Feldman, M. Tomasini, E. Amankwah, J. C. Clare, P. W. Wheeler, D. R. Trainer, and R. S. Whitehouse. A hybrid modular multilevel voltage source converter for hvdc power transmission. IEEE Transactions on Industry Applications, 49(4):1577-1588, July 2013. 
[5] Y. Xue, Z. Xu, and Q. Tu. Modulation and control for a new hybrid cascaded multilevel converter with de blocking capability. IEEE Transactions on Power Delivery, 27(4):2227-2237, Oct 2012.

[6] John Fletcher, Rui Li, Grain Adam, Derrick Holliday, and Barry Williams. Hybrid Cascaded Modular Multilevel Converter for HVDC Transmission Systems: Steady State and DC Fault Ride-Through Capability. IEE Trans. Power Electron., 30(4):1853-1862, 2015.

[7] X. Shi, S. Howell, C. Shumski, S. Filizadeh, and D. Jacobson. Capacitorvoltage regulation and linear-range extension of a hybrid cascaded modular multilevel converter. IET Gener. Transm. Distrib., 11(18):1-22, 2017.

[8] Stephen Jon Finney, Barry Wayne Williams, and Grain Philip Adam. Hybrid converter with ac side cascaded H-bridge cells against H-bridge alternative arm modular multilevel converter: steady-state and dynamic performance. IET Gener. Transm. Distrib., 7(3):318-328, 2013.

[9] Barry W. Williams and Grain Philip Adam. New emerging voltage source converter for high-voltage application: hybrid multilevel converter with dc side H-bridge chain links. IET Gener. Transm. Distrib., 8(4):765-773, 2014.

[10] G. P. Adam, Ibrahim Abdallah Abdelsalam, Khaled Hani Ahmed, and Barry W. Williams. Hybrid multilevel converter with cascaded h-bridge cells for HVDC applications: Operating principle and scalability. IEEE Trans. Power Electron., 30(1):65-77, 2015.

[11] D R Trainer, C C Davidson, C D M Oates, N M Macleod, and D R Critchley. CIGRE 2010 A New Hybrid Voltage-Sourced Converter for HVDC Power Transmission AREVA T \& D HVDC \& FACTS Stafford, UK R. W . CROOKES AREVA T \& D Technology Centre. Converter, 2010. 
[12] M Tomasini, J Clare, P Wheeler, D R Traine, and R S Whitehouset. a Hybrid Voltage Source Converter Arrangement for Hvdc Power Transmission and Reactive Power Compensation. Power Electron. Mach. Drives, pages 1-6, 2010.

[13] E.K. K Amankwah, J.C. C Clare, P.W. W Wheeler, and A.J. J Watson. Cell capacitor voltage control in a parallel hybrid modular multilevel voltage source converter for HVDC applications. 6th IET Int. Conf. Power Electron. Mach. Drives (PEMD 2012), pages E12-E12, 2012.

[14] V.K. Chinnaiyan, J. Jerome, J. Karpagam, and T. Suresh. Control Techniques for Multilevel Voltage Source Inverters. Power Eng. Conf., pages 1023-1028, 2007.

[15] Amankwah K. Emmanuel. A Parallel Hybrid Modular Multilevel Converter for High Voltage DC Applications. Ph.d. dissertation, University of Nottingham, 2013.

[16] Prabha Kundur, Neal J. Balu, and Mark G. Lauby. Power system stability and control. McGraw-hill, New York, 1994.

[17] Mostafa Parniani and Reza Iravani. Computer analysis of small-signal stability of power systems including network dynamics. IEE Proc. - Gener. Transm. Distrib., 142(6):613-617, 1995.

[18] C. Karawita. HVDC Interaction Studies Using Small Signal Stability Assessment. Ph.d. dissertation, University of Manitoba, 2009.

[19] C. Schauder and H. Mehta. Vector analysis and control of advanced static VAR compensators. IEE Proc. C Gener. Transm. Distrib., 140(4):299, 1993. 
[20] G Wu, J Liang, X Zhou, Y Li, A Egea-Alvarez, G Li, H Peng, and X Zhang. Analysis and design of vector control for VSC-HVDC connected to weak grids. CSEE J. Power Energy Syst., 3(2):115-124, 2017.

[21] Lidong Zhang, Lennart Harnefors, and Hans Peter Nee. Powersynchronization control of grid-connected voltage-source converters. IEEE Trans. Power Syst., 25(2):809-820, 2010.

[22] Jenny Z. Zhou, Hui Ding, Shengtao Fan, Yi Zhang, and Aniruddha M. Gole. Impact of short circuit ratio and phase locked loop parameters on the small signal behavior of a VSC HVDC converter. IEEE Trans. Power Deliv., 29 (5):2287-2296, 2014.

[23] A.M. Gole and J.Z. Zhou. VSC transmission limitations imposed by AC system strength and $\mathrm{AC}$ impedance characteristics. 10th IET Int. Conf. AC DC Power Transm. (ACDC 2012), (2):06-06, 2012.

[24] T. Midtsund, J. A. Suul, and T. Undeland. Evaluation of current controller performance and stability for voltage source converters connected to a weak grid. 2nd Int. Symp. Power Electron. Distrib. Gener. Syst. PEDG 2010, pages 382-388, 2010.

[25] Ling Xu and Lingling Fan. Impedance-based resonance analysis in a vsc-hvdc system. IEEE Trans. Power Deliv., 28(4):2209-2216, 2013.

[26] G. Kalcon, G. Adam, O. Anaya-Lara, S. Lo, and K. Uhlen. Small - Signal Stability Analysis of Multi - Terminal VSC - Based DC Transmission Systems. IEEE Trans. Power Syst., 27(4):1818-1830, 2012. 
[27] Jin Huang and Xiaoming Yuan. Impact of the voltage feed-forward and current decoupling on VSC current control stability in weak grid based on complex variables. 2015 IEEE Energy Convers. Congr. Expo. ECCE 2015, pages 6845-6852, 2015.

[28] Mingquan Zhao, Xiaoming Yuan, Jiabing Hu, and Yabing Yan. Voltage Dynamics of Current Control Time-Scale in a VSC-Connected Weak Grid. IEEE Trans. Power Syst., 31(4):2925-2937, 2016.

[29] C. Nanayakkara Yapa, U.D. Annakkage, and S. Filizadeh. Stability Analysis of a Hybrid Modular Multilevel Voltage Source Converter. In 18th Annu. IEEE Canada Electr. Power Energy Conf. Oct. 2018. 


\section{Appendix A}

\section{Co-efficients of the DC system state space model}

Derivation of the co-efficients in order to obtain the state-space model of the DC system for the converter as described in Section.2.2.6 is as given below.

$$
\begin{aligned}
& m_{1}=V_{d c 0}\left(K_{28}-K_{29} x_{c l 0}+K_{30} x_{c a p 0}-K_{31} P_{(r e f)}-K_{32} i_{d c 0}+K_{33} x_{i d c 0}+K_{34}\right) \\
& m_{2}=\dot{V}_{d c 0}\left(K_{28}-K_{29} x_{c l 0}+K_{30} x_{c a p 0}-K_{31} P_{(r e f)}-K_{32} i_{d c 0}+K_{33} x_{i d c 0}+K_{34}\right) \\
& m_{3}=K_{29} \dot{V}_{d c 0} V_{d c 0} \\
& m_{4}=K_{30} \dot{V}_{d c 0} V_{d c 0} \\
& m_{5}=K_{31} \dot{V}_{d c 0} V_{d c 0} \\
& m_{6}=K_{32} \dot{V}_{d c 0} V_{d c 0} \\
& m_{7}=K_{33} \dot{V}_{d c 0} V_{d c 0}
\end{aligned}
$$




$$
\begin{aligned}
& m_{8}=V_{i R 0}\left(K_{1}+K_{2} x_{c l 0}^{2}+K_{3} x_{c a p 0}^{2}+K_{4} P_{(r e f)}^{2}+K_{5} i_{d c 0}^{2}+K_{6} x_{i d c 0}^{2}+V_{G}^{2}-K_{7} x_{c l 0}\right. \\
& +K_{8} x_{c a p 0}-K_{9} P_{(r e f)}-K_{10} i_{d c 0}+K_{11} x_{i d c 0}+K_{12}+x_{c l 0}\left(-K_{13} x_{c a p 0}+K_{14} P_{(r e f)}+K_{15} i_{d c 0}\right. \\
& \left.-K_{16} x_{i d c 0}-K_{17}\right)+x_{c a p 0}\left(-K_{18} P_{(r e f)}-K_{19} i_{d c 0}+K_{20} x_{i d c 0}+K_{21}\right)+P_{(r e f)}\left(K_{22} i_{d c 0}\right. \\
& \left.\left.-K_{23} x_{i d c 0}-K_{24}\right)+i_{d c 0}\left(-K_{25} x_{i d c 0}-K_{26}\right)+K_{27} x_{i d c 0}\right) \\
& m_{9}=V_{i I 0}\left(K_{1}+K_{2} x_{c l 0}^{2}+K_{3} x_{c a p 0}^{2}+K_{4} P_{(r e f)}^{2}+K_{5} i_{d c 0}^{2}+K_{6} x_{i d c 0}^{2}+V_{G}^{2}-K_{7} x_{c l 0}\right. \\
& +K_{8} x_{c a p 0}-K_{9} P_{(r e f)}-K_{10} i_{d c 0}+K_{11} x_{i d c 0}+K_{12}+x_{c l 0}\left(-K_{13} x_{c a p 0}+K_{14} P_{(r e f)}+K_{15} i_{d c 0}\right. \\
& \left.-K_{16} x_{i d c 0}-K_{17}\right)+x_{c a p 0}\left(-K_{18} P_{(r e f)}-K_{19} i_{d c 0}+K_{20} x_{i d c 0}+K_{21}\right)+P_{(r e f)}\left(K_{22} i_{d c 0}\right. \\
& \left.\left.-K_{23} x_{i d c 0}-K_{24}\right)+i_{d c 0}\left(-K_{25} x_{i d c 0}-K_{26}\right)+K_{27} x_{i d c 0}\right) \\
& m_{10}=2 V_{d c 0}\left(-K_{35}+K_{36} x_{c l 0}-K_{37} x_{c a p 0}+K_{38}-K_{39} x_{c l 0}-1.5 K_{40} v_{d c 0}+K_{41}\right. \\
& \left.+K_{42} i_{d c 0}+K_{43}-K_{44} x_{c l 0}+K_{45} x_{c a p 0}-K_{46} P_{(r e f)}-K_{47} i_{d c 0}\right)-i_{d c 0}\left(K_{1}+K_{2} x_{c l 0}^{2}+K_{3} x_{c a p 0}^{2}\right. \\
& +K_{4} P_{(r e f)}^{2}+K_{5} i_{d c 0}^{2}+K_{6} x_{i d c 0}^{2}+V_{G}^{2}-K_{7} x_{c l 0}+K_{8} x_{c a p 0}-K_{9} P_{(r e f)}-K_{10} i_{d c 0} \\
& +K_{11} x_{i d c 0}+K_{12}+x_{c l 0}\left(-K_{13} x_{c a p 0}+K_{14} P_{(r e f)}+K_{15} i_{d c 0}-K_{16} x_{i d c 0}-K_{17}\right) \\
& +x_{c a p 0}\left(-K_{18} P_{(r e f)}-K_{19} i_{d c 0}+K_{20} x_{i d c 0}+K_{21}\right)+P_{(r e f)}\left(K_{22} i_{d c 0}-K_{23} x_{i d c 0}-K_{24}\right) \\
& \left.+i_{d c 0}\left(-K_{25} x_{i d c 0}-K_{26}\right)+K_{27} x_{i d c 0}\right)
\end{aligned}
$$

$$
\begin{gathered}
m_{11}=V_{d c 0}^{2}\left(K_{42}-K_{47}\right)-V_{d c 0}\left(K_{1}+K_{2} x_{c l 0}^{2}+K_{3} x_{c a p 0}^{2}+K_{4} P_{(r e f)}^{2}+3 K_{5} i_{d c 0}^{2}+K_{6} x_{i d c 0}^{2}+V_{G}^{2}\right. \\
-K_{7} x_{c l 0}+K_{8} x_{c a p 0}-K_{9} P_{(r e f)}-2 K_{10} i_{d c 0}+K_{11} x_{i d c 0}+K_{12}+x_{c l 0}\left(-K_{13} x_{c a p 0}+K_{14} P_{(r e f)}\right. \\
\left.+2 K_{15} i_{d c 0}-K_{16} x_{i d c 0}-K_{17}\right)+x_{c a p 0}\left(-K_{18} P_{(r e f)}-2 K_{19} i_{d c 0}+K_{20} x_{i d c 0}+K_{21}\right) \\
\left.+P_{(r e f)}\left(2 K_{22} i_{d c 0}-K_{23} x_{i d c 0}-K_{24}\right)+i_{d c 0}\left(-2 K_{25} x_{i d c 0}-2 K_{26}\right)+K_{27} x_{i d c 0}\right)
\end{gathered}
$$




$$
\begin{array}{r}
m_{12}=V_{d c 0}^{2}\left(K_{36}-K_{39}-K_{44}\right)+V_{i R 0} I_{t R 0}\left(2 K_{2} x_{c l 0}-K_{7}-K_{13} x_{c a p 0}+K_{14} P_{(r e f)}\right. \\
\left.+K_{15} i_{d c 0}-K_{16} x_{i d c 0}-K_{17}\right)+V_{i I 0} I_{t I 0}\left(2 K_{2} x_{c l 0}-K_{7}-K_{13} x_{c a p 0}+K_{14} P_{(r e f)}\right. \\
\left.+K_{15} i_{d c 0}-K_{16} x_{i d c 0}-K_{17}\right)-V_{d c 0} i_{d c 0}\left(2 K_{2} x_{c l 0}-\right. \\
K_{7}-K_{13} x_{c a p 0}+K_{14} P_{(r e f)} \\
\left.+K_{15} i_{d c 0}-K_{16} x_{i d c 0}-K_{17}\right)
\end{array}
$$

$$
\begin{aligned}
m_{13}=V_{d c 0}^{2}\left(-K_{37}+K_{45}\right)+V_{i R 0} I_{t R 0}\left(2 K_{3} x_{c a p 0}+K_{8}-K_{13} x_{c l 0}-K_{18} P_{(r e f)}\right. \\
\left.-K_{19} i_{d c 0}+K_{20} x_{i d c 0}+K_{21}\right)+V_{i I 0} I_{t I 0}\left(2 K_{3} x_{c a p 0}+K_{8}-K_{13} x_{c l 0}-K_{18} P_{(r e f)}\right. \\
\left.-K_{19} i_{d c 0}+K_{20} x_{i d c 0}+K_{21}\right)-V_{d c 0} i_{d c 0}\left(2 K_{3} x_{c a p 0}+K_{8}-K_{13} x_{c l 0}-K_{18} P_{(r e f)}\right. \\
\left.-K_{19} i_{d c 0}+K_{20} x_{i d c 0}+K_{21}\right)
\end{aligned}
$$

$$
\begin{gathered}
m_{14}=V_{i R 0} I_{t R 0}\left(2 K_{5} i_{d c 0}-K_{10}+K_{15} x_{c l 0}-K_{19} x_{c a p 0}+K_{22} P_{(r e f)}-K_{25} x_{i d c 0}-K_{26}\right) \\
+V_{i I 0} I_{t I 0}\left(2 K_{5} i_{d c 0}-K_{10}+K_{15} x_{c l 0}-K_{19} x_{c a p 0}+K_{22} P_{(r e f)}-K_{25} x_{i d c 0}-K_{26}\right)
\end{gathered}
$$

$$
\begin{aligned}
& m_{15}=V_{i R 0} I_{t R 0}\left(2 K_{36} x_{i d c 0}+K_{11}-K_{16} x_{c l 0}+K_{20} x_{c a p 0}-K_{23} P_{(r e f)}-K_{25} i_{d c 0}+K_{27}\right) \\
& +V_{i I 0} I_{t I 0}\left(2 K_{36} x_{i d c 0}+K_{11}-K_{16} x_{c l 0}+K_{20} x_{c a p 0}-K_{23} P_{(r e f)}-K_{25} i_{d c 0}+K_{27}\right) \\
& -V_{d c 0} i_{d c 0}\left(2 K_{36} x_{i d c 0}+K_{11}-K_{16} x_{c l 0}+K_{20} x_{c a p 0}-K_{23} P_{(r e f)}-K_{25} i_{d c 0}+K_{27}\right)
\end{aligned}
$$

$$
\begin{aligned}
& m_{16}=-K_{46} V_{d c 0}^{2}+V_{i R 0} I_{t R 0}\left(2 K_{4} P_{(r e f)}-K_{9}+K_{14} x_{c l 0}-K_{18} x_{c a p 0}+K_{2} 2 i_{d c 0}-K_{23} x_{i d c 0}-K_{24}\right) \\
& +V_{i I 0} I_{t I 0}\left(2 K_{4} P_{(r e f)}-K_{9}+K_{14} x_{c l 0}-K_{18} x_{c a p 0}+K_{2} 2 i_{d c 0}-K_{23} x_{i d c 0}-K_{24}\right) \\
& -V_{d c 0} I_{d c 0}\left(2 K_{4} P_{(r e f)}-K_{9}+K_{14} x_{c l 0}-K_{18} x_{c a p 0}+K_{2} 2 i_{d c 0}-K_{23} x_{i d c 0}-K_{24}\right)
\end{aligned}
$$




$$
\begin{array}{r}
m_{17}=I_{t R 0}\left(K_{1}+K_{2} x_{c l 0}^{2}+K_{3} x_{c a p 0}^{2}+K_{4} P_{(r e f)}^{2}+K_{5} i_{d c 0}^{2}+K_{6} x_{i d c 0}^{2}+V_{G}^{2}-K_{7} x_{c l 0}\right. \\
+K_{8} x_{c a p 0}-K_{9} P_{(r e f)}-K_{10} i_{d c 0}+K_{11} x_{i d c 0}+K_{12}+x_{c l 0}\left(-K_{13} x_{c a p 0}+K_{14} P_{(r e f)}+K_{15} i_{d c 0}\right. \\
\left.-K_{16} x_{i d c 0}-K_{17}\right)+x_{c a p 0}\left(-K_{18} P_{(r e f)}-K_{19} i_{d c 0}+K_{20} x_{i d c 0}+K_{21}\right)+P_{(r e f)}\left(K_{22} i_{d c 0}\right. \\
\left.\left.-K_{23} x_{i d c 0}-K_{24}\right)+i_{d c 0}\left(-K_{25} x_{i d c 0}-K_{26}\right)+K_{27} x_{i d c 0}\right) \\
+K_{8} x_{c a p 0}-K_{9} P_{(r e f)}-K_{10} i_{d c 0}+K_{11} x_{i d c 0}+K_{12}+x_{c l 0}\left(-K_{13} x_{c a p 0}+K_{14} P_{(r e f)}+K_{15} i_{d c 0}\right. \\
\left.-K_{16} x_{i d c 0}-K_{17}\right)+x_{c a p 0}\left(-K_{18} P_{(r e f)}-K_{19} i_{d c 0}+K_{20} x_{i d c 0}+K_{21}\right)+P_{(r e f)}\left(K_{22} i_{d c 0}\right. \\
\left.\left.-K_{23} x_{i d c 0}-K_{24}\right)+i_{d c 0}\left(-K_{25} x_{i d c 0}-K_{26}\right)+K_{27} x_{i d c 0}\right)
\end{array}
$$

where

$$
\begin{aligned}
K_{1} & =\frac{\left(3 n_{c} v_{c a p(r e f)}^{2} k_{3} k_{4} K_{P i d c} K_{P c l}\right)^{2}}{V_{G}^{2}} \\
K_{2} & =\frac{\left(3 n_{c} k_{3} k_{4} K_{P i d c} K_{P c l}\right)^{2}}{V_{G}^{2}} \\
K_{3} & =\frac{\left(k_{3} k_{4} K_{P i d c} K_{I c l}\right)^{2}}{V_{G}^{2}} \\
K_{4} & =\frac{\left(k_{4} K_{P i d c}\right)^{2}}{V_{G}^{2}} \\
K_{5} & =\left(k_{4} K_{P i d c}\right)^{2} \\
K_{6} & =\left(k_{4} K_{I i d c}\right)^{2} \\
K_{7} & =\frac{6 n_{c}\left(k_{3} k_{4}\right)^{2} K_{P i d c}^{2} K_{P c l}^{2} v_{c a p(r e f)}^{2}}{V_{G}^{2}} \\
K_{8} & =\frac{6 n_{c}\left(k_{3} k_{4}\right)^{2} K_{P i d c}^{2} K_{P c l} K_{I c l} v_{c a p(r e f)}^{2}}{V_{G}^{2}} \\
K_{9} & =\frac{6 n_{c} k_{3}\left(k_{4}\right)^{2} K_{P i d c}^{2} K_{P c l} v_{c a p(r e f)}^{2}}{V_{G}^{2}}
\end{aligned}
$$




$$
\begin{aligned}
& K_{10}=\frac{6 n_{c} k_{3}\left(k_{4}\right)^{2} K_{P i d c}^{2} K_{P c l} v_{c a p(r e f)}^{2}}{V_{G}} \\
& K_{11}=\frac{6 n_{c} k_{3}\left(k_{4}\right)^{2} K_{P i d c} K_{P c l} K_{I i d c} v_{c a p(r e f)}^{2}}{V_{G}} \\
& K_{12}=6 n_{c} k_{3} k_{4} K_{P i d c} K_{P c l} v_{c a p(r e f)}^{2} \\
& K_{13}=\frac{2\left(k_{3} k_{4}\right)^{2} K_{P i d c}^{2} K_{P c l} K_{I c l}}{V_{G}^{2}} \\
& K_{14}=\frac{2 k_{3}\left(k_{4}\right)^{2} K_{P i d c}^{2} K_{P c l}}{V_{G}^{2}} \\
& K_{15}=\frac{2 k_{3}\left(k_{4}\right)^{2} K_{P i d c}^{2} K_{P c l}}{V_{G}} \\
& K_{16}=\frac{2 k_{3}\left(k_{4}\right)^{2} K_{P i d c} K_{P c l} K_{I i d c}}{V_{G}} \\
& K_{17}=2 k_{3} k_{4} K_{P i d c}^{2} K_{P c l} \\
& K_{18}=\frac{2 k_{3}\left(k_{4}\right)^{2} K_{P i d c}^{2} K_{I c l}}{V_{G}^{2}} \\
& K_{19}=\frac{2 k_{3}\left(k_{4}\right)^{2} K_{P i d c}^{2} K_{I c l}}{V_{G}} \\
& K_{20}=\frac{2 k_{3}\left(k_{4}\right)^{2} K_{P i d c} K_{\text {Icl }} K_{I i d c}}{V_{G}} \\
& K_{21}=2 k_{3} k_{4} K_{\text {Pidc }} K_{\text {Icl }} \\
& K_{22}=\frac{2\left(k_{4}\right)^{2} K_{P i d c}^{2}}{V_{G}} \\
& K_{23}=\frac{2\left(k_{4}\right)^{2} K_{\text {Pidc }} K_{\text {Iidc }}}{V_{G}} \\
& K_{24}=2 k_{4} K_{P i d c} \\
& K_{25}=2\left(k_{4}\right)^{2} K_{P i d c} K_{\text {Iidc }} \\
& K_{26}=2 k_{4} K_{P i d c} V_{G} \\
& K_{27}=2 k_{4} K_{\text {Iidc }} V_{G} \\
& K_{28}=\frac{3 n_{c} v_{c a p(r e f)}^{3} C_{c e l l} k_{3} k_{4} K_{P i d c} K_{P c l}}{V_{G}^{2}} \\
& K_{29}=\frac{v_{c a p(r e f)} C_{c e l l} k_{3} k_{4} K_{P i d c} K_{P c l}}{V_{G}^{2}} \\
& K_{30}=\frac{v_{\text {cap }(\text { ref })} C_{c e l l} k_{3} k_{4} K_{\text {Pidc }} K_{\text {Icl }}}{V_{G}^{2}}
\end{aligned}
$$




$$
\begin{aligned}
& K_{31}=\frac{v_{\text {cap }(r e f)} C_{c e l l} k_{4} K_{P i d c}}{V_{G}^{2}} \\
& K_{32}=v_{\text {cap (ref })} C_{\text {cell }} k_{4} K_{\text {Pidc }} \\
& K_{33}=v_{\text {cap }(r e f)} C_{c e l l} k_{4} K_{\text {Iidc }} \\
& K_{34}=v_{\text {cap }(r e f)} C_{\text {cell }} V_{G} \\
& K_{35}=\frac{3 n_{c} v_{c a p(r e f)}^{3} C_{c e l l} k_{3} k_{4} K_{P i d c} K_{P c l}^{2}}{\left(2 V_{G}\right)} \\
& K_{36}=\frac{v_{c a p(r e f)} k_{3} k_{4} K_{P i d c} K_{P c l}^{2}}{\left(2 V_{G}\right)} \\
& K_{37}=\frac{v_{c a p(r e f)} k_{3} k_{4} K_{P i d c} K_{P c l} K_{I c l}}{\left(2 V_{G}\right)} \\
& K_{38}=\frac{3 n_{c} v_{c a p(r e f)}^{3} C_{c e l l} k_{3} k_{4} K_{\text {Pidc }} K_{\text {Icl }}}{2 V_{G}} \\
& K_{39}=\frac{v_{\text {cap }(r e f)} C_{c e l l} k_{3} k_{4} K_{\text {Pidc }} K_{\text {Icl }}}{2 V_{G}} \\
& K_{40}=\frac{v_{c a p(r e f)} C_{c e l l} k_{4} K_{P i d c} \omega_{0}}{2 L_{d c}} \\
& K_{41}=\frac{v_{\text {cap }(r e f)} C_{\text {cell }} k_{4} K_{\text {Pidc }} \omega_{0} V_{G}}{2 L_{d c}} \\
& K_{42}=\frac{v_{\text {cap (ref) }} C_{c e l l} k_{4} K_{P i d c} \omega_{0} R_{d c}}{2 L_{d c}} \\
& K_{43}=\frac{3 n_{c} v_{c a p(r e f)}^{3} C_{c e l l} k_{3} k_{4} K_{P c l} K_{I d c}}{2 V_{G}} \\
& K_{44}=\frac{v_{c a p(r e f)} C_{c e l l} k_{3} k_{4} K_{P c l} K_{I d c}}{2 V_{G}} \\
& K_{45}=\frac{v_{\text {cap (ref) }} C_{c e l l} k_{3} k_{4} K_{I c l} K_{I d c}}{2 V_{G}} \\
& K_{46}=\frac{v_{\text {cap }(r e f)} C_{c e l l} k_{4} K_{I d c}}{2 V_{G}} \\
& K_{47}=\frac{v_{c a p(r e f)} C_{c e l l} k_{4} K_{I c l} K_{I d c}}{2} \\
& k_{4}=\frac{I_{d c(\text { base })}}{V_{d c(\text { base })}}
\end{aligned}
$$

